Abstracts*

\title{
9th Santorini Conference Systems Medicine and Personalised Health \& Therapy
}

Santorini, Greece, 30 September - 3 October 2018

\section{Committees}

\section{Steering Committee}

Sofia Siest, Nancy, France, President of the Santorini conferences

John Lamont, Co Antrim, United Kingdom

Michael Marschler, Mannheim, Germany

Robert Barouki, Paris, France

Athanase Benetos, Nancy, France

Georges Dagher, Paris, France

Panagiotis Deloukas, Cambridge, United Kingdom

\section{Scientific Advisory Board}

Sofia Siest, Nancy, France, President of the Santorini conferences

Robert Barouki, Paris, France

Athanase Benetos, Nancy, France

Georges Dagher, Paris, France

Panos Deloukas, Cambridge, United Kingdom

John Lamont, Co Antrim, United Kingdom

Michael Marschler, Mannheim, Germany

Urs Meyer, Basel, Switzerland

Charity Nofziger, Salzburg, Austria

Marcus Paulmichl, Salzburg, Austria

Lynn Webster, Salt Lake City, United States

\section{Organizing Committee}

\section{UMR INSERM U1122; IGE-PCV "Interactions Gène-Environnement} en Physiopathologie Cardio-Vasculaire, Nancy, France"

Siest Sofia, Gorenjak Vesna, Hiegel Brigitte, Masson Christine, Stathopoulou Maria, Petrelis Alexander, Weryha Georges Richard

*These abstracts have been reproduced directly from the material supplied by the authors, without editorial alteration by the staff of this Journal. Insufficiencies of preparation, grammar, spelling, style, syntax, and usage are the authors. 


\title{
Sunday 30, September
}

\section{3:00 pm - 05:00 pm - CLOSED MEETING - MAST4HEALTH}

With the participation of:

Georges Dedoussis, Athens, Greece, Panagiotis Deloukas, London, United Kingdom, Chiara

Barbieri, Pisa, Italy, Natasa Milic, Novi Sad, Serbia, Maria Stathopoulou, Nancy, France, Sofia

Siest, Nancy, France

\section{WELCOME}

\section{Sofia Siest}

UMR INSERM U1122; IGE-PCV, University of Lorraine, Nancy, France

Sophia SIEST will have the first word to introduce the $9^{\text {th }}$ Santorini Conference.

She will make a brief summary about how has this colloquium, initiated in 2002 and as such being the oldest International conference (held every two years) in the field of personalised medicine and pharmacogenomics, evolved in the past 16 years.

Eight "Santorini Conferences" welcomed worldwide distinguished speakers and attendees in a world of passion of science with specific Personalised Medicine sessions on Genetics and Pharmacogenomics. Over the years, the "Santorini Conferences" became one of the most important conferences on genetic predisposition to health, diseases, response to drugs and environment.

After 16 years, the $\mathbf{9}^{\text {th }}$ Santorini Conference "Systems medicine and Personalised Health and Therapy" aims to reflect on the frontiers of current genomic knowledge in the fields of cardio-metabolic diseases and cancer and to reveal the practical use of this knowledge in preventive, diagnostic and pharmacogenomic ways to directly impact the socio-economic aspects of public health.

With her presentation inspired by the Odyssey, Greek ancient epic poem of Homer, she will embark with the attendees on a ship from Troy-Hope to Ithaca-Practice, coursing through the history of the momentous events and achievements that paved the way for personalised medicine.

She will set sail amidst important genetic discoveries and obstacles slowing the potential full implementation of accumulated knowledge into everyday practice, beginning with the discovery of the first human genome and voyaging through the projects that contributed to the progress of personalised medicine.

\section{KEYNOTE LECTURE}

\section{The journey to personalised medicine - landmarks and challenges}

\section{Eric Boerwinkle}

UTHealth School of Public Health, Houston, United States

No abstract available

\section{Monday 1st, October}

\section{Session I - ‘-OMICS’ BIOMARKERS COMMONALITY IN CARDIO-METABOLIC DISEASES AND CANCER - CAN SEQUENCING OFFER A DIAGNOSIS?}

\author{
Metabolomics applied to novel cardiometabolic disease mechanisms and therapeutic targets
}

P J White ${ }^{1}$, R W McGarrah ${ }^{1}$, MA Herman ${ }^{1}$, DT Chuang ${ }^{2}$, CB Newgard $^{1}$

${ }^{1}$ Duke Molecular Physiology Institute, Duke University Medical Center, Durham, NC USA; ${ }^{2}$ Department of Biochemistry, University of Texas Southwestern Medical Center, Dallas, TX USA

Background: We have applied metabolomics and other "omics" tools for understanding of mechanisms contributing to pandemic metabolic diseases of our era--diabetes, obesity, and cardiovascular disease, as well as novel interventional targets. We have been investigating the 
mechanistic and therapeutic significance of a metabolomic signature of perturbed branched chain amino acid (BCAA) catabolism in multiple cohorts of insulin resistant humans compared to normally insulin sensitive controls (reviewed in 1). Several studies have demonstrated the prognostic power of this signature to predict incident diabetes and intervention outcomes. These metabolites are also uniquely sensitive to the most efficacious interventions for obesity and diabetes.

Objective and Design: To investigate the potential role of BCAA in cardiometabolic diseases, we have used rodent models to demonstrate that BCAA supplementation exacerbates insulin resistance, whereas BCAA restriction in Zucker fatty rats (ZFR) improves insulin sensitivity. Tissue amino acid and lipidomic profiles suggest that relief of mitochondrial fuel overload serves as a contributing mechanism for the salutary effect of BCAA restriction. From a therapeutic perspective, we have tested the impact of stimulation of BCAA catabolism by activation of the branched-chain ketoacid dehydrogenase (BCKDH) complex by small molecule inhibition of the kinase (BDK) or overexpression of the phosphatase (PPM1K) that regulate its activity.

Results: Small molecule inhibition of BDK, or overexpression of PPM1K, improves glucose, lipid and amino acid homeostasis in ZFR, including enhancement of insulin sensitivity and lowering of liver triglycerides. Phosphoproteomics analysis identified ATP-citrate lyase (ACL) as an alternative BDK/PPM1K substrate. Overexpression of BDK is sufficient to phosphorylate and activate ACL, leading to increased hepatic de novo lipogenesis. Finally, BDK is upregulated and PPM1K downregulated by fructose feeding and the ChREBP-b transcription factor.

Conclusions: These studies identify a new ChREBP-regulated mechanism that links BCAA, glucose and lipid metabolism. Intervention at this node reverses several obesity-associated metabolic disease phenotypes. Overall, this work demonstrates the potential of metabolic profiling for defining novel metabolic disease mechanisms and new therapeutic strategies.

Acknowledgements: Supported by NIH grant PO1 DK58398 (to CBN) and a Pathways to Stop Diabetes Award from the American Diabetes Association (to PJW).

Reference: Newgard CB. Cell Metabolism 2017; 25: 43-56.

\title{
The 100,000 Genomes Project- transforming genomics in healthcare
}

\author{
Mark Caulfield \\ The William Harvey Research Institute, London, United Kingdom \\ No abstract available
}

\section{The International Cancer Genome project}

\section{Peter Campbell}

The Wellcome Sanger Institute, London, United Kingdom

No abstract available

\section{Personalised tumor markers}

\author{
Eleftherios Diamandis \\ Laboratory Medicine \& Pathobiology, Mount Sinai Hospital, Toronto, CANADA
}

Background: The cancer biomarker discovery pipeline is progressing slowly. The difficulties of finding novel and effective biomarkers for diagnosis and management of cancer patients are well-known. We speculate that it is unlikely to discover new serological biomarkers characterized by high sensitivity and specificity. This projection is supported by recent findings that cancers are genetically highly heterogeneous. Objective: We propose a new way of improving the landscape of cancer biomarker research. There are currently hundreds, if not thousands, of described biomarkers which perform at high specificity (>90\%), but at relatively low sensitivity $(<30 \%)$. We call these "rare tumor markers." Borrowing from the principles of precision medicine, we advocate that among these low sensitivity markers, some may be useful for specific patients.

Design and Results: We suggest screening new patients for hundreds to thousands of cancer biomarkers, to identify a few that are informative, and then use them clinically. This is similar to what is currently done with genomics, to identify personalized therapies. We further suggest this approach may explain as to why some biomarkers are elevated in only a small group of patients. It is likely that these differences in expression are linked to specific genomic alterations which could then be found with genomic sequencing.

Conclusions: Clinically weak global biomarkers may play important roles for managing individual cancer patients, especially in this era of numerous new therapies in clinical trials. More specifically, circulating tumor DNA, one of the best personalized cancer biomarkers, may play important roles in prognosis and therapy selection and monitoring. Its value as an early cancer detection marker is still under intense investigation

References: Kulasingam V, Prassas I, Diamandis EP. Npj Precis Oncol 2017;1:17-20. 


\title{
Session II - UNMET CLINICAL NEEDS IN THE PREVENTION AND TREATMENT OF CARDIO-METABOLIC DISEASES AND CANCER - COMORBIDITIES
}

\author{
Epigenetics of Cardiovascular Diseases
}

\author{
Manuel Rosa Garrido \\ David Geffen School of Medicine at UCLA, Los Angeles, United States
}

Background: In the USA alone, over 8 million people will suffer from heart failure by the year 2030. Development of heart failure is a long and complex process which combines cellular, metabolic, electrophysiological, calcium handling and epigenetic changes to culminate in pathophysiological cardiac structure and function. Epigenetic mechanisms control gene expression at the individual locus scale (e.g. histone post-translational modifications and DNA methylation) but also at the genomic scale via the formation of long range regulatory interactions and the formation of chromosome territories.

Objective: To determine the influence of epigenetics during the development of heart failure.

Design: We performed chromatin capture (Hi-C), reduced representational bisulfite sequencing (RRBS) and RNA-seq experiments comparing control, transverse aortic constriction (TAC) mice (a pressure overload model that induces heart failure) and CTCF null mice (chromatin structural protein involved in the regulation of higher order chromatin structure and maintenance of local three-dimensional chromatin structure). Results: Our Hi-C experiments demonstrated that global remodeling of chromatin after TAC and CTCF depletion drives heart failure. These experiments showed that $4 \%$ of genes undergo a change in active versus inactive compartmentalization, including genes involved in heart disease during cardiac disease. Integrated analysis of Hi-C and RNA-seq data demonstrate that differentially expressed genes after heart failure tend to physically interact with genes that are co-regulated in the same direction. Analyses of histone marks in these gene neighborhoods reveal the relative enrichment of activating and inhibiting marks, H3K27ac and H3K27me3 respectively. Our RRBS experiments also show that $99 \%$ of the shared differentially methylated CpGs in TAC and CTCF-KO share the same direction of change in the specialized chromatin environments associated with genes, suggesting that heart failure has a conserved epigenetic component operative during development of the disease that involves DNA methylation.

Conclusions: Our data suggest that genes exist within structural microenvironments, wherein histone marks and DNA methylation play an important role in controlling expression. Heart failure involves conserved structural reprogramming of these chromatin microenvironments.

\section{Telomere length and the Cancer/Atherosclerosis trade off}

\author{
Abraham Aviv \\ Rutgers, The State University of New Jersey, New Jersey Medical School, Center of Human Development and Aging, Newark
}

Evolutionary theories posit that comparatively short telomere length (TL) and consequently curtailed replicative potential promote resistance to cancer by attenuating the accumulation of driver mutations in somatic tissues of long-lived mammals, including humans. The trade-off, however, is a propensity to aging-related degenerative diseases because of compromised replicative potential in older animals. The cancerdegenerative disease trade-off might apply not only across mammalian species, but also across humans.

In the general population, short leukocyte TL (LTL) is associated with atherosclerotic cardiovascular disease (CVD), while long LTL is associated with several major cancers. These associations manifest at the genomic level, as SNPs associated with short LTL are also associated with CVD. In contrast, SNPs associated with long LTL are also associated with several cancers. The majority of these SNPs are mapped to genetic loci that harbor known TL maintenance genes, including hTERT and TERC, the genes encoding the two subunits of telomerase- the reverse transcriptase that adds telomere repeats to the ends of the chromosomes.

Jointly, these findings suggest that TL might play a causal role in CVD and some cancers, since a) having short or long TL precedes by decades the manifestations of these two disease categories, and $b$ ) the associations of variant TL maintenance genes with CVD and cancer principally exclude reverse causality, i.e., CVD shortens while cancer lengthens LTL. These findings also have considerable ramifications for longevity of contemporary humans. 


\title{
ORAL COMMUNICATIONS SESSION
}

\section{Genetic and protein profiling of cancer tumours, a first step towards personalised therapy}

\author{
Said El Shamieh \\ Medical Laboratory Sciences, Beirut Arab University, Beirut, Lebanon
}

Background: Next generation sequencing (NGS) along with immunohistochemistry (IHC) is currently used in the cancer field to decipher molecular markers that may be translated to new diagnostic, prognostic and therapeutic targets.

Methods: NGS analysis of 592 genes along with IHC was performed on tumour samples of an aggressive form of triple negative breast cancer (TNBC) and an endometrioid endometrial carcinoma (ECC) case.

Results: Whereas sequencing of TNBC tumour revealed RICTOR gene amplification along with a mutated p53, sequencing of ECC revealed mutated PTEN and PIK3CA genes (p.R173C and p.H1047R respectively). IHC showed increased PD-1 protein levels in ECC. Following ECC profiling, everolimus treatment was prescribed; this is based on previous work showing that individuals having H1047R mutation in PIK3CA can benefit from mTOR inhibitors. Despite these observations, our case did not show any signs of improvement. Since the tumour was positive for PD-1, pembrolizumab treatment following the everolimus one resulted to a significant decrease of tumour size. A patient-tailored therapy couldn't be applied on our TNBC case, and the disease progressed aggressively suggesting that RICTOR promotes the genesis of TNBC and supporting existing evidence that RICTOR plays a role in tumor progression and poor prognosis of breast cancer.

Conclusion: Our results have deciphered possible genetic and protein biomarkers implicated in the aetiology and progression of cancer. Furthermore, in this work we established a guideline for a personalised treatment in ECC.

\section{Clock/clock related genes analysis in sleep problems in an adult population with autism and intellectual disability}

\author{
Ana Peiro \\ Clinical Pharmacology, HGUA, Spain
}

Background: Sleep problems are the most prevalent comorbidity in adults with autism and intellectual disability. This could be due to multifactorial causes, including genetic alterations in melatonin pathway and clock genes, that can play an important role in these sleep problems mainly insomnia and circadian rhythm sleep-wake disorders

Purpose: Analyze the influence of NPAS, Per1, ASMT and MTNR1A gene variants in the presence of sleep problems in adults on the autism spectrum

Methods: The influence of the genotype was analyzed according to sleep problems in 120 individuals with autism and intellectual disability by DSM5 diagnosis. Ambulatory circadian monitoring (ACM, 24 hours for 1 week) and sleep diaries were used to evaluate sleep parameters. Nonparametric circadian rhythm indexes were analysed as secondary outcome measures. Drugs prescribed and adverse events were recorded. Data were statistically analysed with R 3.2.4 software.

Results: Subjects (29 \pm 11 years old, 83\% males, body mass index (BMI) $26.5 \pm 6.4 \mathrm{Kg} / \mathrm{m} 2$ ) presented mainly insomnia (61-91\%). Analysis per genotype showed significant differences for Per1 and ASMT genes with a significantly higher number of awakenings in GG individuals. Allelic analysis showed that individuals carrying the G allele of Per1 gene presented significantly shorter sleep onset latency, and lesser number of awakenings.

Conclusions: Then, the relationships between disrupted sleep, mainly insomnia symptoms, is present in this population. Further research may open interesting perspectives for early detection of potential sleep problems according to the genes and further therapeutic interventions. 


\title{
Welcome to a new world of Intelligent Connected Care
}

\author{
Lunzenfichter Gilles \\ Medisanté AG, Luzern, Switzerland
}

Short Description: Most nations face a daunting challenge. Chronic conditions already represent almost $80 \%$ of their healthcare budget, and they are still on the rise! Therefore, the leaders of the national healthcare systems realize that a digital re-think of the care continuum and reimbursement policies is required to allow a connected care model alongside an in-person care model.

Introduction, Motivation: A wealth of connected devices with mobile apps, mostly in the well-being area, already connects consumers to their health. But, this patient-generated health data (PGHD) is not available to their caregivers and is stored in global clouds. Therefore, caregivers cannot react in a timely manner if vital signs of a remote diabetic or hypertensive patient deteriorate and this may result in costly hospital readmissions. Today, the expertise of caregivers only applies when patients visit them; it does not extend beyond the 4 walls of their care center.

Material, Methods, Tools: Medisanté connects caregivers to patient-generated health data (PGHD) for chronic conditions such as diabetes, hypertension, cardiovascular and overweight indications - timely and seamlessly, through an unique combination of Devices with 3G Connectivity, a Connected Care Platform and In-Country Data Hosting.

Description of the solution: Medisanté delivers a turnkey connected care solution to caregivers with actionable insights across multiple conditions. Combined with a seamless end-to-end integration from patient devices to the ELIOT platform and secure in-country data hosting, it helps nations deliver a new model of care at a fraction of current costs.

Results: Experience and lessons learned Partnership with NHS: Medisanté has been listed as supplier in an announcement of the National Health Service (NHS) in England. Thanks to the existing contract, a first pilot project for the telemonitoring of hypertonic patients through physicians has been started at the end of 2017 by the NHS Partnership with Vodafone: We are officially IoT Partner of Vodafone and have been invited to their booth at the Medica 2017 trade fair. Their worldwide M2M network and IoT SIM-cards are at the heart of our Connected Care Service Partnership with HPE: We were invited at their EMEA User Conference, HP Discover 2017, in Madrid as an IoT-Partner in healthcarePartnership with Siemens Healthineers: They offered us a global marketing collaboration agreement after Arab Health and will embark us in many major tradeshows (HIMSS, ConhIT, ...). Outlook. We want to become the leading provider in Healthcare IoT.

\section{Temporal comorbidity patterns in prostate cancer disease trajectories based on semantic, phenotypic and genetic similarities}

A Giannoula, E Centeno, F Sanz and LI Furlong

Hospital del Mar Medical Research Institute, Pompeu Fabra University, Barcelona, Spain

Background: Prostate cancer is the most common neoplasm in men and the third most frequent cause of cancer death in Europe. One of the most critical problems that patients and physicians face is deciding about screening and treatment. Central to clinical decision making is the presence of comorbidities, as the selected treatment plan may exacerbate comorbid diseases and inversely, certain comorbid diseases may negatively affect the treatment outcome. A deeper understanding of prostate cancer comorbidities is important for the optimal disease management and patients' survival.

Objective: This work aims at performing a population-based comorbidity study on prostate cancer patients from an epidemiological, genetic and phenotypic perspective, by also taking the time factor into account. Time is crucial in assessing comorbidities, as it permits to identify complex disease associations and study disease progression1.

Design: The clinical histories of 21,309 hospitalized prostate cancer patients are extracted from a Catalonian health registry and shared disease trajectories of various lengths are identified. Subsequently, an unsupervised clustering algorithm is applied, based on Dynamic Time Warping (DTW), that groups trajectories according to temporal characteristics that they share, irrespectively of the length, duration and time scale. Three distance metrics are proposed for calculating disease similarities in DTW: a semantic similarity metric based on the Unified Medical Language System topology, a genetic similarity metric that uses the Jaccard index on shared gene sets and a phenotypic similarity one based on the Human Phenotype Ontology.

Results: More than 2,000 prostate-cancer common disease trajectories were identified and clustered, according to shared semantic, genetic and phenotypic characteristics. Various prostate cancer comorbidities could be retrieved and their time dynamics could be observed at all disease levels. Among the most frequent ones, were diabetes, cardiovascular and respiratory disease, while other less frequent clusters involved, for example, time-dependent associations between prostate cancer and dementia, septicemia, osteopathies, etc.

Conclusions: Temporal disease patterns were identified in prostate-cancer trajectories that reflected, either, semantic, genetic or phenotypic similarities between diseases. These results can serve as the preliminary basis for understanding prostate cancer and its comorbidities, thereby, facilitating the development of survival prediction models. 


\section{Investigation of novel biomarkers of drug-induced kidney injury in renal transplant recipients undergoing graft biopsy}

Laurent Becquemont ${ }^{1}$, Michon Arthur ${ }^{2}$, Antoine Durrbach ${ }^{3}$, Jean-Charles Gautier ${ }^{4}$, Xavier Benain ${ }^{5}$, Catherine Lunven ${ }^{6}$, Alexandre Jagerschmidt 7 , Catherine Aubert ${ }^{8}$, Oliver Poetz ${ }^{9}$, Thomas Joos ${ }^{9}$, Thierry Gury ${ }^{4}$,

1. Pharmacology, University paris orsay, Kremlin bicêtre, France

2. Nephrology, APHP, Kremlin bicêtre, France

3. Nephrology, University paris orsay, Kremlin bicêtre, France

4. Preclinical safety, SANOFI R\&D, Vitry-sur-Seine, France

5. Biostatistics and Programming, SANOFI R\&D, Montpellier, France

6. Clinical Sciences and Operations, SANOFI R\&D, Chilly-Mazarin, France

7. Translational Medicine and Early Development, SANOFI R\&D, Chilly-Mazarin, France

8. Biomarkers and Clinical Bioanalyses, Translational Medicine and Early Development, SANOFI R\&D, Vitry-sur-Seine, France

9. Natural and Medical Sciences Institute, University Tübingen, Reutlingen, Germany

Introduction: Present urinary and blood kidney biomarkers (BM) remain insufficient to detect early kidney injury. Our aim was to compare new kidney BM with histopathological data in kidney allograft recipients.

Patients and methods: Consecutive adult patients were included between 2011 and 2015, Blood and urine samples were collected just prior graft biopsy. All kidney samples were classified according to Banff 2007. The diagnostic performance (area under ROC curve, AUROC) of 146 new BM was compared to those of classical BM (total urinary proteins, blood urea nitrogen, eGFR and serum creatinine) to correctly identify histopathological groups.

Results: From 257 included patients, 223 could be analyzed. Versus slightly modified renal parenchyma (SMRP), microalbuminuria and total urinary proteins had the highest diagnostic performance toward glomerular injury, with an AUROC of 0.98 (95\%CI 0.93-1) and 0.97 (95\%CI 0.92-1) respectively. Similarly, urinary neutrophil gelatinase associated lipocalin (NGAL) had the best performance values reflecting acute tubular necrosis (ATN) with an AUROC of 0.93 (95\%CI 0.85-0.99) (for comparison serum creatinine and BUN had AUROC of 0.87 and 0.88 , respectively). During early transplantation, urinary NGAL had the best performance values to discriminate ATN from acute rejection with an AUROC of 0.85 (95\%CI 0.70-0.95), while combinations of BM including NGAL reached an AUROC of 0.94 (95\%CI 0.83-1).

Conclusion: During the initial phase of transplantation, combination of urinary NGAL to additional BM might be helpful to discriminate between ATN and acute graft rejection.

Keywords: Biomarkers, renal transplantation, renal graft injury, renal graft biopsy

\section{Molecular taxonomy of sporadic amyotrophic lateral sclerosis using disease-associated genes}

Cavallaro Sebastiano, Morello Giovanna, Spampinato Antonio G.

Institute of Neurological Sciences-CNR, Catania, ITALY

Background: Advances in diagnostic techniques and high-throughput biotechnologies are giving a compelling opportunity to improve the diagnosis and treatment of disease by developing a "New Taxonomy" that defines diseases on the basis of their underlying molecular and environmental factors, rather than on traditional physical signs and symptoms. Oncology represents the first interesting example of how genomic medicine has changed disease understanding and therapy. However, much work remains to be done on molecular characterization and classification of complex and multifactorial diseases, including neurodegenerative disorders. In the last years, our research group has taken important steps towards the development of a new approach to the classification of neurodegenerative diseases, highlighting for the first time the existence of a biological and molecular heterogeneity of amyotrophic lateral sclerosis (ALS) a severe clinical condition characterized by upper and lower motor neuron degeneration for which there is no truly effective treatment (1).

Objective: Rather than approaching ALS as a single entity, we advocate targeting therapies to distinct "clusters" of patients based on their specific genomic and molecular features.

Design: In our previous work, comprehensive genomic profiling of 41 motor cortex samples enabled to discriminate control from sporadic ALS (SALS) patients and segregated these latter in two distinct subgroups, each associated with different deregulated genes and pathways. Interestingly, some deregulated genes in sporadic ALS were previously associated to familiar ALS, indicating shared pathogenic mechanisms between the two forms of disease. In the present study, we performed cluster analysis on the same whole-genome expression profiles using a restricted (203) subset of genes extensively implicated in monogenic forms of ALS.

Results: Surprisingly, this short and unbiased gene list was sufficiently representative to accurately differentiate SALS patients from controls, suggesting that sporadic and monogenic forms of ALS share common etiopathogenic mechanisms. In addition, the same unbiased gene list confirmed the stratification of SALS patients into two molecularly distinct subgroups associated with a good number of cluster-specific candidate genes and unique biological features.

Conclusions: Overall, our findings support the existence of a molecular taxonomy for ALS and represent a further step towards the establishment of a molecular-based diagnosis and patient-tailored therapies. 


\title{
Session III - PIPELINE CHALLENGES IN PRECISION MEDICINE - FROM PATIENT SAMPLING TO A CLINICAL REPORT
}

\author{
From Patient sampling to clinical report - The Austrian experience
}

\author{
Markus Paulmichl \\ Salzburg, Austria \\ No abstract available
}

\author{
Standardized improved pre-analytical workflows: the bridge to good quality samples for reliable analytical test \\ results
}

\author{
Uwe Oelmueller \\ Molecular Diagnostics, QIAGEN GmbH, Hilden,
}

Background: Molecular in vitro diagnostics and biomedical research have allowed great progress in medicine. Further progress is expected by new biomarker tests analyzing specimens biomolecule profiles such as nucleic acids, proteins, and metabolites. However, profiles of these molecules can change significantly during specimen collection, transport, storage, and processing, caused by post collection cellular changes such as gene inductions, gene down regulations, biomolecules modifications or degradation. This can make the outcome from diagnostics or research unreliable or even impossible because the analytical test will not determine the situation in the patient body but an artificial specimen analyte profile generated during the pre-analytical workflow. High quality specimens with preserved analyte profiles are therefore crucial to biobanking, research and diagnostics.

Objective: New pre-analytical workflow technologies and international standards are needed for securing good quality clinical specimens. Design: The EU funded the SPIDIA consortium (2008-2013) with 16 academic institutions, international organisations and private companies for driving the standardisation and improvement of pre-analytical procedures for in-vitro diagnostics. The new EU SPIDIA4P project (2017-2020) builds on SPIDIA's results. It is supported by19 partners from private industry, public institutions and one European Standards Organisation (www.spidia.eu).

Results: SPIDIA could achieve significant progress by developing new pre-analytical workflow technologies. Within pan-European ring trials, SPIDIA could also generate broad evidence that guidance to laboratories on pre-analytical workflows parameters improves analytical test results. Based on this and other evidence, new standard documents were developed at the European Committee for Standardization (CEN). The CEN/Technical Committee "In vitro Diagnostic Medical Devices" has released first 9 Technical Specifications to standardize pre-analytical workflows for different blood, other body fluids and tissue based molecular applications. They are currently progressing to International Standards at the ISO/Technical Committee "Clinical Laboratory Testing and In Vitro Diagnostic Test Systems".

The new SPIDA4P consortium project (2017-2020) has started to broaden this portfolio by generating and implementing finally a portfolio of 22 pre-analytical CEN/Technical Specifications and ISO/International Standards, addressing important pre-analytical workflows for personalized medicine. Corresponding External Quality Assurance (EQA) Schemes are under development as well.

Acknowledgement: The SPIDIA project has received funding under the Seventh Research Framework Program of the European Union under grant agreement no. 222916. The SPIDIA4P project receives funding from the European Union's Horizon 2020 research and innovation program under grant agreement no. 733112.

\section{Using tissue samples for quantitative protein and phosphoprotein analysis - critical considerations}

\author{
Karl Friedrich Becker \\ Technical University of Munich, Pathology, Munich, Germany
}

Access to high quality human tissue samples is one of the most important prerequisites for modern biomedical research, including the analysis of proteins and phosphoproteins. Collecting human tissue samples is a complex process that varies between hospitals or even within single institutions. The influences of sample processing, including sample collection, transport, stabilization, storage and analyte (e.g. protein) extraction, on the final assay result are not very well recognized in pre-clinical and clinical research laboratories, the daily routine clinical processes, biobanks, and in physicians' offices. In the current workflows the laboratories performing the molecular assay usually are not aware of what happed to the sample before the analytical test is performed. Therefore, standardization of the entire workflow from test ordering to the report of the molecular assay, with special emphasis on the pre-analytical phase, is crucial for successful integration of proteomic studies in the clinic as protein and phosphoprotein profiles may change due to sample processing. The aim of this presentation is to highlight the progress of proteomic studies with human tissues and to provide an overview of current international activities to standardize the pre-analytical phase by drafting pre-analytical CEN/TS and ISO/IS documents, focusing on the European projects SPIDIA and SPIDIA4P. 


\title{
Challenges in using liquid biopsies for precision medicine
}

Maria G. Daidone

Fondazione IRCCS, Istituto Nazionale dei Tumori, Milan, Italy

No abstract available

\section{Session IV - PGX - REGULATORY PERSPECTIVES AND FUTURE TECHNICAL/ANALYTICAL DEVELOPMENTS}

\author{
EMA Good Pharmacogenomic Practice (GPP) group EMA: Pharmacogenomics Working Party: Relevant new Guidelines
}

\author{
Markus Paulmichl \\ Salzburg, Austria \\ No abstract available
}

\author{
Upcoming challenges in PGx - plasmonic biosensing for liquid biopsy applications: the ULTRAPLACAD platform \\ G Spoto \\ I.N.B.B. Consortium, Catania, Italy. Department of chemical sciences, University of Catania, Catania, Italy.
}

Background: Standard methods for the detection of circulating nucleic acids exploit the enzymatic amplification of the target sequences to achieve the required limit of detection. The amplification step introduces drawbacks and can generate artefacts as a consequence of sample contamination and recombination between homologous regions of DNA. Advanced methods for the detection of biomarkers are requested to operate with high selectivity, multiplexed capability and ultrasensitivity. In this context, efforts have been made to identify innovative PCRfree methods for the detection of circulating nucleic acids.

ULTRAPLACAD is an EU Horizon 2020 funded project aimed at developing an innovative plasmonic platform for the ultrasensitive detection of both circulating nucleic acids (both DNA mutation and microRNAs) and protein biomarkers.

Objective: The plasmonic detection of nucleic acids will be discussed in the context of the ULTRAPLACAD project with particular attention to opportunities offered by the nanoparticle-enhanced surface plasmon resonance imaging for the PCR-free detection of nucleic acids. The aim of the ULTRAPLACAD platform will be presented by highlighting the specific capability to detect both nucleic acid and protein biomarkers.

Results: The ultrasensitive PCR-free plasmonic detection of point mutations in genomic DNA will be presented. The ULTRAPLACAD industrial prototype will be introduced and main features of the new platform will be discussed in the context of colorectal cancer liquid biopsy application.

Conclusions: ULTRAPLACAD is aiming at providing a new platform for the plasmonic detection of circulating biomarkers. The platform is available as an industrial prototype and detects with enhanced sensitivity both nucleic acids and proteins.

Acknowledgements: The EU Horizon 2020 project ULTRAPLACAD (n. 633937) is acknowledge for financial support.

\section{Tuesday 2 October}

\section{Session V - GENE-ENVIRONMENT INTERACTIONS IN CARDIO-METABOLIC DISEASES AND CANCER}

\author{
Nutrigenetics in Non-Alcoholic Fatty Liver Disease
}

Georges Dedoussis

University of Harokopio, Athens, Greece

Non-Alcoholic Fatty Liver Disease (NAFLD) is nowadays considered a complex disease, resulting from a variety of genetic and environmental factors. It is characterized by excessive flux of fatty acids (FA) and triglycerides (TG) accumulation in the liver, primarily activated by dietary factors, obesity and insulin resistance. In addition to the established risk factors, such as age, BMI and gender, a polygenic background is believed to confer susceptibility to the onset and progression of NAFLD. To date, there are four genetic variants that have been identified as 
risk factors for NAFLD: PNPLA3 rs738409, TM6SF2 rs58542926, MBOAT7 rs641738 and GCKR rs780094. This multiple-hit model of NAFLD pathogenesis indicates the complexity of the disease and highlights the need to control liver and systemic inflammation. As mentioned above, diet is a major contributor to the pathogenesis of NAFLD but it is also an integral part of its treatment. Overall, maintaining a normal body weight and a balanced diet has been proved to reduce inflammation and promote help, while excessive energy intake is a key determinant of the disease onset and progression. Increased consumption of fat, especially saturated fatty acids, and simple sugars have also been positively associated with the disease. On the other hand, energy restriction, high protein, high n-3 polyunsaturated fatty acids (PUFA) and antioxidants intake have been inversely associated with hepatic steatosis. Considering the multifactorial background and the various gene-environment interactions that lead to NAFLD onset and progression, we and others have studied gene-diet interactions. Such efforts have recently revealed, that in response to high Fish intake, carriers of a TM6SF2 variant, experienced increased intrahepatic accumulation of TG and, therefore, hepatic steatosis (1). Similar data reported a lower response of PNPLA3 risk allele carriers to DHA supplementation in children with NAFLD. Unraveling gene-diet interactions in NAFLD onset and progression constitute the key to apply personalized nutrition and, therefore, provide patients with more efficient therapies.

\title{
Gene-environment interactions in cardio-vascular diseases: '-omics' and systems biology
}

\section{Myriam Fornage}

University of Texas Health Science Center at Houston, McGovern Medical School, Institute of Molecular Medicine, Research Center for Human Genetics, Houston, United States

No abstract available

\section{A perspective on interaction effects in genetic association studies - from detection to risk prediction}

\author{
H Aschard ${ }^{1,2}$ \\ ${ }^{1}$ Centre de Bioinformatique, Biostatistique et Biologie Intégrative (C3BI), Institut Pasteur, Paris, France. \\ ${ }^{2}$ Department of Epidemiology, Harvard TH Chan School of Public Health, Boston, Massachusetts, USA.
}

Background: The identification of gene-environment interaction in human traits and diseases is an active area of research that generates high expectation, and most often lead to high disappointment. This is partly explained by a misunderstanding of the inherent characteristics of standard regression-based interaction analyses.

Objective: Here, I revisit and untangle theoretical aspects of interaction tests in regression models. In particular, I discuss variables coding scheme, interpretation of effect estimate, statistical power, and outcome prediction in regard of various hypothetical interaction patterns. I further use real data example, including breast cancer, to illustrate the potential impact in terms of detection and clinical utility.

Results: I first show that the simplest biological interaction models-in which the magnitude of a genetic effect depends on a common exposure-are among the most difficult to identify. Second, I highlight the demerit of the current strategy to evaluate the contribution of interaction effects to quantitative and binary outcomes, and argue for the use of new approaches to overcome this issue. Third, I demonstrate the advantages and limitations of multivariate interaction models, when testing for interaction involving multiple SNPs and/or multiple exposures. Finally, I illustrate how the later multivariate approaches can allow the identification of subgroups in the population whose genetic background makes them more susceptible to the deleterious effects of environmental exposure.

Conclusions: Together, these new insights can be leveraged for future method development and to improve our understanding of the interplay between genetic variants and environmental exposures in multifactorial traits and diseases.

\section{Exposome/epigenomics and clinical application}

\section{R Barouki}

Inserm UMR-S 1124, Université Paris Descartes, and Hôpital Necker Enfants maladies, Paris France robert.barouki@parisdescartes.fr

Background: Identifying precise and predictive biomarkers of health and disease is a critical objective of clinical biochemistry and biomedical research. New concepts and technologies have emerged recently that could support such an objective, notably the exposome concept which corresponds to the totality of exposures over the lifetime from conception to death.

Objective: The aim of the presentation is to show how recent developments in exposome studies and epigenomics could be used to support preventive action in medicine

Recent developments: Research in the exposome field allowed the development of sensors and biological biomarkers using omics technologies that can support the prediction of the effect of those exposures on human health. For example, authors have used metabolomics 
to explore the chemical exposome and its initial effects on organisms. Recently, epigenomics has proven to be of great value to characterize environmental effects. Indeed, several environmental factors such as chemicals, nutritional unbalance and psychological stress can influence epigenetic regulation and this may account for their long-term effects since those marks could last for decades and possibly for generations.

While precision medicine has primarily focused on adapting treatments to the genetic profiles of tumors, it had in fact originally a wider scope including the use of robust biomarkers for disease prevention. Using different sensors, metabolomics and epigenomics it seems now possible to generate precise observations that could be of value for prevention.

Conclusion: The integration of the genome, epigenome and exposome data can be useful in medicine. Such an integrated view will help strengthen approaches to identify relevant predictive markers that can support precise prevention actions both at the population and at the individual levels.

Reference: R Barouki et al, Epigenetics as a mechanism linking developmental exposures to long-term toxicity. Env Int 114 (2018) $77-86$

\title{
Session VI - LATEST INSIGHTS IN STROKE: CLINICAL TRIALS AND APPLICABILITY OF ‘-OMICS’ DATA IN PATIENT'S STRATIFICATION
}

\author{
Stroke pharmacogenetics: from hope to reality
}

\author{
Guillaume Pare \\ McMaster University Department: Pathology and Molecular Medicine, Hamilton, Ontario, Canada
}

In the United States alone, it is expected that more than 690,000 patients will have an ischemic stroke while 240,000 patients will have a transient ischemic attack (TIA) in each year. The use of antiplatelet and anticoagulant therapies has lowered the risk of recurrent strokes but response to treatment varies from one individual to the other. There has also been an increased understanding of stroke pharmacogenetics which has been driven by advances in genotyping technology and increased interest in developing targeted pharmaceutical treatments. The primary goal of pharmacogenetics is to treat patients who are more likely to benefit from a particular intervention by using their genetic information to select appropriate treatments. However, implementation of pharmacogenetics in clinical settings remains limited both because of lack of awareness and need for evidence-based recommendations. This review will summarize the pharmacogenetics of stroke by providing an overview of the genetic variants that contribute to the individual responses to aspirin, clopidogrel, warfarin and dabigatran, as well as discuss considerations for evidence-based implementation.

\section{Protein biomarkers and diagnosis of ischemic stroke}

\section{Konstantinos Makris ${ }^{1}$ and $\underline{\text { Alexander Haliassos }}^{1}$}

1. Clinical Biochemistry Department, KAT General Hospital, Kifissia, Athens, Greece and 2. ESEAP Proficiency Testing Scheme for Clinical Laboratories, Athens, Greece.

Fast and accurate diagnosis of stroke is crucial for the immediate application of the right therapy to patients. However, rapid diagnosis is still a challenge since an ischemic stroke cannot be identified based only on clinical assessment. CT or MRI imaging is required to rule out hemorrhagic stroke since thrombolytic therapy can lead to increased intracranial bleeding and further aggravation of hemorrhagic stroke. In addition, clinical situations that imitate the signs and symptoms of stroke may also impede the rapid diagnosis and treatment of stroke victims. It is therefore of value to discover non-invasive tests that aim to quickly distinguish stroke from stroke mimics and distinguish ischemic from hemorrhagic stroke. Identifying blood biomarkers of stroke is an active area of research since their potential use is not limited to diagnosis and differentiation, but can be applied to prognosis and patient monitoring - monitoring the effectiveness of applied therapy and/or diagnose possible complications. However, their use has been limited so far not only for reasons related to patients and the disease (heterogeneity of stroke etiology, the complexity of the ischemic cascade, the impact of the blood-brain barrier (BBB) on diffusion of blood biomarkers, and difficulties in obtaining consent from stroke patients), but also for reasons that related to laboratory measurement of these biomarkers (preanalytical and analytical issues as well as interpretation of laboratory measurements). Until today, many biomarkers have been identified; however none so far have shown sufficient sensitivity and specificity in order to be used in the clinical setting. In this presentation, we will focus on ischemic stroke and we aim to highlight these problems and also investigate if these are due to stroke complexity or due to our limited knowledge of preanalytical requirements for many of these molecules and the questionable quality of the assays used.

Reference: Konstantinos Makris, Alexander Haliassos, Maria Chondrogianni \& Georgios Tsivgoulis (2018). Blood biomarkers in ischemic stroke: potential role and challenges in clinical practice and research, Critical Reviews in Clinical Laboratory Sciences, DOI: 10.1080/10408363.2018.1461190. 


\title{
ILLUMINA WORKSHOP - PHARMACOGENOMICS FOR PRE-EMPTIVE SCREENING
}

\author{
Pharmacogenetics using the Illumina GSA array
}

Ron Van Schaik

Rotterdam, The Netherlands

Estonian personalised medicine initiative: polygenic risk scores, pharmacogenetics and rare mutations.

Lili Milani

Tartu, Estonia

\section{Session VII - PAIN MANAGEMENT - A 'JOURNEY' FROM ‘CLINICAL TRIAL' VIA 'POST- MARKETING PHARMACOVIGILANCE/RISK MANAGEMENT’ TO 'SUCCESS STORY'}

\author{
Genetic biomarkers as common denominators for specific pain disorders
}

\author{
L Webster \\ PRA Health Sciences, Salt Lake City, UT, USA.
}

Background: Chronic pain affects about $20 \%$ of the population in developed countries. Of all the chronic pain conditions, chronic low back pain (CLBP) is one of the most common pain disorders that results in enormous fiscal burden on patients and societies. CLBP is characterized by different phenotypes with different underlying molecular pathologies complicated by a multitude of psychosocial factors. Unfortunately, there is little known about omics (glycomics, Activomics and Geonme-wide association studies-GWAS) biomarkers that contribute to the risk of developing and severity of CLBP.

Objective: To describe a study that is part of the PainOMICS project funded by European Community in the Seventh Framework Programme (FP7)-THEME (HEALTH.2013.2.2.1-5-Understanding and controlling pain) with the goal to determine ' -omics' biomarkers (GWAS, glycomics and Activomics) potentially associated with susceptibility to CLBP and with different pathophysiological patterns. An additional study objective is to recognize genetic variants associated with persistent CLBP, by comparing patients having CLBP and pain-free patients.

Design: The study is a retrospective observational, multicenter, international clinical study, with a case- control design. The project includes six clinical centers from Italy, Croatia, Belgium, Australia and the USA, and four centers for scientific analyses from Croatia, France, Germany and UK. Statistical expertise is provided by 'PolyOmica' consulting, based in the Netherlands. All participants will have detailed pain and health histories. Blood samples for -omics analyses were taken from each enrolled patient at the time of the enrolment, and biological samples were sent to analytical partners of the consortium for specific -omics analysis.

Results: No results have been published.

Conclusions: This appears to be the first study to investigate genetic and -omic biomarkers in a large population sample of CLPB patients. The researchers anticipate the biomarkers will show a relationship to pain sensation, as well as to disease pathophysiology and pain generators. If successful, the results of the study could lead to more personalized therapy and preventive measures for high risk individuals.

Acknowledgements: Massimo A, Manuela DG, Cristina ME, Catherine K, Wei W, Moira S, Christian G, Judith M, Iain PK, Jane M, Frances, MK W, Jan VZ, Klaas B, Gordan L, Ivan G, Dragan P, Andrea S, Yurii AS, Lennart KC, Leonardo K, Richard R, Guido F, BMJ Open 2016;6:e012070. doi:10.1136/bmjopen-2016-012070.

\section{Pharmacogenetics of opioid treatments}

\section{Laure Elens}

Catholic University of Louvain, Louvain-la-Neuve, Belgium

Description: Pain is an important multifactorial mechanism for human defense that is affected by a combination of physical, environmental, cognitive, emotional and genetic factors. Some individuals are more sensitive to pain and this could be very problematic, especially during medical interventions. Opioids are potent analgesic agents commonly used as effective pre-medication for the treatment of pain. However, using the same analgesic treatment, inter-individual differences in sensitivity to pain and opioid analgesic effects (toxicity and inefficacy) are well described making it difficult to manage discomfort during and after medical intervention or for certain painful disorders (e.g. cancer pain management). Some evidences suggest that genetic variations and single nucleotide polymorphisms (SNPs) in genes implicated in the opioid response pathway or the mechanisms of pain sensing might explain the various phenotypes related to pain susceptibility and response to opioid therapy. This presentation will try to summarize current knowledge in opiod pharmacogenetics and the possible beneficial applications that it could have in clinical practice to allow better individualization of pain discomfort when treated with opioids. 
Learning Objectives: To review the current knowledge of the genetics of opioid sensitivity and metabolism

To make an overview of opioid pharmacogenetic studies and how it might be useful in understanding inter-individual differences in pain perception and treatment effectiveness and/or drug related-toxicity.

To determine whether genetic testing has any clinical significance in decision making for the management and control of pain.

\title{
Pharmacogenomics in post-marketing pharmacovigilance
}

\section{Noelia Martin Granado}

Senior Safety Scientist, Pharmacovigilance and Patient Safety, PRA Health Sciences, RGES Spain

Background: Pharmacovigilance is the science and activities relating to the detection, assessment, understanding and prevention of Adverse Drug Reactions (ADRs). ADRs are referred to as any response to a drug which is noxious and unintended. There is robust evidence that genomic factors may play a key role in the pathogenesis of both predictable and idiosyncratic ADRs. Indeed over the past years, several studies have identified a number of common and rare variants that are associated with an increased risk of ADRs. In particular, polymorphisms in genes encoding drug transporters, drug-metabolizing enzymes and drug targets (e.g. enzymes, receptors) can lead to the occurrence of ADRs. As more reliable and affordable genetic testing tools become available pharmacogenomics seems promising to facilitate personalized drug therapy by maximizing the therapeutic efficacy of drugs while minimizing the occurrence of ADRs in patients. Due to the strong synergy existing between pharmacovigilance and pharmacogenomics disciplines, as both aim to understand the inter-relationships between drug therapeutic efficacy and safety, it has been proposed to put into practice pharmacogenovigilance: pharmacovigilance activities informed and guided by accompanying pharmacogenomics analyses.

Objective: To review the potential for developing specific pharmacogenovigilance programs by integrating pharmacogenomics with pharmacovigilance (e.g. risk management, signal detection and product labelling) in the setting of real-world post-marketing pharmacovigilance supported by adequate evidence-focused scientific literature review.

Design: Targeted scientific literature review results accompanied by the outcomes of the PRA Pharmacogenomics Working Group experience will be evaluated to ascertain whether the development of tailored pharmacogenovigilance programs can improve significantly clinical practice nowadays.

Results: An increased number of genetically bound incidences of drug-related issues lead to the delayed detection and under-reporting of ADRs. Moreover it was discovered that genetic variations significantly influences pharmacokinetics and pharmacodynamics. Pharmacogenetics tests have been developed and made available in clinical practice to safeguard patients from ADRs. Furthermore, drugs frequently associated with ADRs are known to be metabolized by enzyme with genetic polymorphism.

Conclusions: Despite several challenges still need to be faced, the great potential of pharmacogenomics in reducing the occurrence of ADRs justifies the need of developing new pharmacogenovigilance strategies to better personalize drug treatment, to refine the current post-marketing drug safety surveillance and to optimally safeguard patient health.

Acknowledgements: PRA Health Science Pharmacogenomics Working Group Chairs and Members.

\section{The adoption of Pharmacogenomics leading to patient benefit}

\author{
Andrew Purchase \\ Prahealthsciences, Swansea, United Kingdom
}

In this presentation Andrew Purchase, Senior Safety Scientist, will provide an overview of the increasing acceptance and adoption of Pharmacogenomics into clinical practice, and how this translates into benefits for the patient. The presentation will also explore methodologies for raising patient awareness and understanding of the potential benefits linked to Pharmacogenomics, as well as current/future technologies that will support the use of genomic data.

\section{ORAL COMMUNICATIONS SESSION}

\section{Genomic Medicine and Individualized Approaches in Africa: Challenges and Opportunities}

\author{
Ibrahim Muntaser \\ Unit of Disease and Diversity, Institute of Endemic Diseases, University of Khartoum, Khartoum, SUDAN
}

Human variations, both in their genetic and phenotypic facets were historically referenced against a background of non-African genomes and traits, mainly as a matter of convenience. However, following the realization of a large African effective population size and its ancestral nature, an urge to study the various repercussions of such large effective size on human health has ensued particularly in Africa. Genetic 
diversity after all is the ultimate outcome of both population and gene histories, and being as such, a wider ramification into medical and health intervention, inspired concepts of population and individualized medicine that is now progressively gaining grounds instead of a conventional simplistic universal approach to health. We present examples of relevance to such challenges and opportunities that may lie ahead of genetic research and populations studies in Africa for instance a) reasons for poor performance of conventional approaches to genetic mapping of traits in Africa b) some peculiar features of high effective size that affects personalized diagnosis e.g the excess of minor reference alleles c) the multiplicity of genetic causations resulting in non-straight forward genotype phenotype relationship including pharmacogenomic associations in individuals and populations.

\title{
Bridging the Multi-Omics Precision Medicine Gap in the Middle East: The Valdia Health Experience
}

\author{
Carlos Malpica \\ CEO, Valdia Health, Doha, QATAR
}

Real prevention medicine is now possible. Providing actionable information to clinicians, in time to prevent the onset of disease, is made possible by increased health knowledge using multiple omic tools. Valdia Health is a privately-owned company Technology established in Doha, Qatar. The company specializes in best-in-class diagnostic support in precision medicine with individual omic data, adding value to new generation diagnostics. Qatar is well positioned to implement Precision Medicine approaches to benefit its citizens and health care system, translating omic tools into clinical practice. Following the example of Qatar, the company aims to accelerate the path towards precision medicine in the Middle East.

\section{Dark Quencher Dyes Useful for Hyper-Sensitive Fluorescent Molecular probes. New Versatile Approach in qPCR Probe Design}

\author{
Andrei Laikhter \\ Oligonucleotide synthesis, Chemgenes, Wilmington, United States
}

Several methods of oligonucleotide labeling techniques have been employed in the oligonucleotide based probe synthesis. The most critical issue in this technology is the quality of the corresponding building blocks containing the reporter molecule. A novel method utilizing "Click Chemistry" and 2'/3'-alkynyl modified nucleosides has been developed at ChemGenes Corporation. That approach allows the synthesis of multiply labeled oligonucleotides in combinatorial fashion. Using that method and recently developed novel quencher dyes Instant Quenchers (IQ2 and IQ4) Chemgenes Corporations offers highly efficient and reliable qPCR probes.

\section{Evolution of dihydropyrimidine dehydrogenase (DPD) diagnostics in a single center in a time-period of eight years}

BJC van den Bosch ${ }^{1}$, ADC Paulussen ${ }^{1}, M_{\text {Breuer }}^{1}$, M Lindhout $^{1}$, DCJ Tserpelis ${ }^{1}$, A Steyls ${ }^{1}, J_{\text {Bierau }}^{1}$, MJH Coenen ${ }^{2}$ 1 Department of Clinical Genetics, Maastricht University Medical Center + (MUMC+), Maastricht, The Netherlands 2 Department of Human Genetics, Radboud University Medical Center, Radboud Institute for Health Sciences, Nijmegen, The Netherlands

Background: Treatment with fluoropyrimidines, the main chemotherapeutic agents used in many types of cancer, is not well tolerated in a subgroup of patients. The enzyme responsible for degradation of fluoropyrimidines is dihydropyrimidine dehydrogenase (DPD), the first and rate-limiting enzyme of the pyrimidine degradation pathway. DPD dysfunction leads to an increased exposure of active metabolites, which can result in severe or even fatal toxicity.

Objective: Fluoropyrimidine treatment can be optimized based on DPD activity.

Design: We provide an overview of eight years DPD diagnostics $(n=1194)$. In this time-frame the test has evolved from a single enzyme measurement using Ultra-High Performance Liquid chromatography (UHPLC) in peripheral blood mononuclear cells (PBMCs) to a combined enzymatic and genetic test of four variants in the DPYD gene (DPYD*2A, DPYD^13, c.2846A > T and 1129-5923C $>$ G).

Results: In the group tested for four variants $(n=814)$, patients with either one variant have a lower enzyme activity than the overall patient group. The majority of patients with the DPYD`2A variant (83\%) consistently showed a decreased enzyme activity. Only seventeen (22\%) of the 77 patients with a low enzyme activity (tested for four variants) carried a variant. Complete DPYD sequencing in a subgroup with low enzyme activity and without $\mathrm{DPYD}{ }^{\star} 2 \mathrm{~A}$ variant $(\mathrm{n}=47)$ revealed 10 genetic variants, of which four have not been described previously.

Conclusions: We did not observe a strong link between DPYD genotype and enzyme activity. There is no doubt that DPD status should be determined before treatment with fluoropyrimidines to save patients' lives and prevent unnecessary side effects. Our study in combination 
with literature shows that there is a discrepancy between the DPD enzyme activity and the presence of clinically relevant SNPs. Therefore at this moment a combination of a genetic and enzymatic test is preferable for diagnostic testing.

\title{
CD8(+) and PD-L1(+) cell densities in biopsies may predict response to Durvalumab in NSCLC patients
}

\author{
Sonja Althammer \\ Bioinformatics, Definiens, Munich, Germany
}

Content: Predicting response to immunotherapies is an active area of cancer research. While the manually determined PD-L1 tumor cell status enriches for patients responding to anti-PD1/PD-L1 treatments, a more accurate predictive signature is needed. We previously reported that CD8 combined with PD-L1 provided an improved means of predicting response to anti-PD-L1 therapy in Non-Small-Cell-Lung-Cancer (NSCLC) patients than PD-L1 alone. Here, we extend this line of study by adjusting for potential confounders in the statistical analysis.

Technology: Digital slides were fully automatically scored using the product of CD8(+) and PD-L1(+) cell densities with the Definiens' Developer XD Software.

Design: Baseline tumor biopsies were analyzed for PD-L1 (Ventana SP263) and CD8 (Ventana SP239) by immunohistochemistry. Samples were taken from patients with advanced NSCLC enrolled in Study NCT01693562, a nonrandomized phase 1/2 trial evaluating Durvalumab, an $\alpha$-PD-L1 monoclonal antibody, ( $\mathrm{N}=163$, split into train and test set). In additional to these Durvalumab treated patients we also analyzed samples from NSCLC patients (T2+ T3) who received chemotherapy $(\mathrm{N}=134)$. Multi-variate Cox analysis was deployed to adjust for potential confounders while comparing signature candidates.

Results: The product of CD8(+) and PD-L1(+) cell densities provided greater overall survival stratification $(\mathrm{p}=0.0001 \mathrm{on}$ test set) than high cell densities of CD8(+) or PD-L1(+), and of the manually determined PD-L1 tumor cell status for NSCLC patients who received $\alpha$-PD-L1. This signature was not prognostic for survival of patients treated with chemotherapy $(p=0.6)$.

Conclusions: Automated digital scoring of tumor biopsy immunohistochemistry can be a useful part of the clinical management of NSCLC patients who might receive immunotherapy. Combining CD8 and PD-L1 data into a single immunohistochemistry signature provides greater predictive value than the prevailing manual PD-L1 assay alone. The lack of prognostic significance of this signature further supports its predictive utility.

\section{Wednesday 3 October}

\section{Session VIII - PHARMACOGENOMICS: CHALLENGES OF CLINICAL TRANSLATION}

\author{
Safety of drug treatment in oncology: a genomic perspective
}

\author{
F Innocenti \\ Eshelman School of Pharmacy, Center for Pharmacogenomics and Individualized Therapy, University of North Carolina Chapel Hill, USA
}

By putting the patient, rather than the disease, at the center of personalized care, the concept of safety gains a much wider connotation. Hippocrate's "primum non nocere", "first, do not harm" remains a fundamental guiding principle of any intervention in medical care. The suffering and discomfort experienced by patients because of the symptoms related to the adverse events of drug treatment should never be discounted. Adverse events have a significant burden on the quality of life of the patient and the families. They reduce confidence in the treatment and might demoralize the patient and the trust on the efficacy of therapies that should improve patient well-being, not reducing it. And we should not forget that, in this era of advanced technologies integrated into patient care, patients treated with anti-cancer therapies are still at risk of losing their lives as a result of the medication that should treat their cancers. Severe adverse events often lead to permanent discontinuation of therapy. Mild-to-moderate toxicities, in addition to affecting quality of life, can also reduce the intensity of the regimen. Most cancer patients receive multi-drug regimens, and toxicity from one drug can halt also the whole regimen. When the goal of treatment is to maintain acceptable quality of life and prolong survivorship (or survival), patient discomfort (from gastrointestinal effects) or cosmetic changes (acne or skin rash), for example, even when they are not severe, might reduce adherence to oral therapy, increasing the chance of recurrence of disease. This presentation will describe the place for genetic analyses to increase patient safety, and how they have the potential of improving safety of cancer drugs in several ways. These aspects are of particular importance for implementation, as access to genetic profiling is becoming more common for patients.

Reference: Innocenti F. Bringing a genomic perspective to the safety of drug treatment in oncology.F1000Res. 2017 Mar 29;6. pii: F1000 Faculty Rev-385. doi: 10.12688/f1000research.10475.1. eCollection 2017. Review. PMID:28408974. 


\title{
Technical Challenges in CYP2D6 analysis
}

\author{
Charity Nofziger \\ PharmGenetix Gmbh, Vienna, Austria
}

Scientists in the field of PGx, as well as physicians and analytical laboratories, are constantly in the search for efficient and affordable methodologies for assessing patient genotypes; and as one would expect, both commercial and private entities are continuously designing and validating these very types of techniques. However, limitations come with every methodology, no matter the cost, robustness or extent of validation. One such limitation includes allele drop-out events, due to the presence of genetic variations besides the one of interest, which, for example, have been described for multiple genotyping assays (1). While the majority of these interfering variants are rare (occurring at a frequency of less than $1 \%$ in the population), they can severely alter the prediction of metabolizing phenotypes within a particular patient. CYP2D6 is responsible for metabolizing around $25 \%$ of clinically prescribed drugs and is riddled with function altering variations including point mutations, insertions and deletions, as well as hybrid formation with its psuedogene, CYP2D7-all of which make accurate genotyping quite challenging (2). Not surprisingly, numerous allele drop out events have been described for popular CYP2D6 genotyping assays (3-7). Here we describe the nature of the latest documented allele drop out event for CYP2D6, involving the CYP2D6*3 allele.

References:

1 Hahn S, Garvin AM, Di Naro E, Holzgreve W. Allele drop-out can occur in alleles differing by a single nucleotide and is not alleviated by preamplification or minor template increments. Genet Test 1998; 2:351-355.

2 Nofziger C, Paulmichl M. Accurately genotyping CYP2D6: not for the faint of heart. Pharmacogenomics 2018.

3 Gaedigk A, Freeman N, Hartshorne T, Riffel AK, Irwin D, Bishop JR et al. SNP genotyping using TaqMan technology: the CYP2D6*17 assay conundrum. Sci Rep 2015; 5:9257.

4 Gaedigk A, Riffel AK, Leeder JS. CYP2D6 Haplotype Determination Using Long Range Allele-Specific Amplification: Resolution of a Complex Genotype and a Discordant Genotype Involving the CYP2D6^59 Allele. J Mol Diagn 2015; 17:740-748.

5 Rasmussen HB, Werge T. Novel variant of CYP2D6* 6 is undetected by a commonly used genotyping procedure. Pharmacol Rep 2011; 63:1264-1266. 6 Riffel AK, Dehghani M, Hartshorne T, Floyd KC, Leeder JS, Rosenblatt KP et al. CYP2D7 Sequence Variation Interferes with TaqMan CYP2D6 $\left.{ }^{\star}\right) 15$ and $\left(^{\star}\right) 35$ Genotyping. Front Pharmacol 2015; 6:312.

7 Scantamburlo G, Tziolia K, Zopf M, Bernardinelli E, Soyal SM, Civello DA et al. Allele Drop Out Conferred by a Frequent CYP2D6 Genetic Variation For Commonly Used CYP2D6³ Genotyping Assays. Cell Physiol Biochem 2017; 43:2297-2309.

\section{Pharmacogenomics - a paradigm for digital medicine}

\section{Urs A Meyer}

Biozentrum, University of Basel, 4056 Basel, Switzerland

Approximately $15 \%$ of drug labels approved by FDA and EMA contain pharmaco-genomic information. Multiple variants of $\sim 20$ germline genes affecting $~ 90$ drugs are presently considered as clinically actionable, i.e. may imply a change in standard therapy. Another development is the growing number of somatically acquired mutations in cancer cells which direct the development and selection of "targeted" anticancer drugs for individual patients. The number of actionable genomic information for drug therapy is limited. In most therapies, efficacy and toxicity is determined by the combined action of multiple genetic, epigenetic, environmental and host factors, and the genetic contribution may be too small for predicting drug response. In this context, the application of omics-based data in combination with clinical and environmental factors to predict an individual's drug response is one of the visions of pharmacogenomics (PGx), personalized medicine and precision medicine. New generation sequencing and microarrays enable accurate and inexpensive measurement of all presently known genetic components of interindividual variability in drug response. However, clinical implementation of pharmacogenomic testing has not yet been widely adopted to improve routine patient care. One reason is that in order to guide prescribing decisions, preemptive pharmacogenomic test results should be available to physicians at the point of care.For an increasing number of actionable gene-drug interactions, practice guidelines based on genetic and clinical information have been established (1). The incorporation of pharmacogenetic test results combined with clinical decision support (CDS) into machine-readable electronic medical records (EMRs) allows clinical implementation of this information. Results of preemptive testing in several institutions with multiple pharmacogenes and "high risk" drugs indicates that > $95 \%$ of patients carry at least one actionable "high risk" genotype. The lecture presents an overview of present studies of clinical implementation of digital signatures of the drug response profiles and discusses how to best apply the already existing digital genomic and other omics information to optimize drug response. 1) Clinical Pharmacogenetics Implementation Consortium: http://cpicpgx.org

\section{Implementation of Pharmacogenetics in Turkish Clinical Practice: The Example of Dihydropyrimidine Dehydrogenase}

\author{
Belgin Süsleyici \\ Molecular Biolgy, Marmara University, Istanbul, TURKEY
}

Fluorouracil is a chemotherapy agent that belongs to the drug class Fluoropyrimidines. The DPYD gene encodes Dihydropyrimidine Dehydrogenase (DPD), an enzyme catalyzing the rate limiting step in fluorouracil metabolism. Colorectal cancer is the most common cause of cancer-related 
death, with about two million new cases being diagnosed each year and the incidence of disease increasing by a year. The most important problems encountered in the treatment of colorectal adenocarcinoma are the side effects of chemotherapy drugs and the failure of chemotherapy due to drug resistance. Although the drug resistance potential is very high, 5 -fluorouracil (5-FU) is the most common chemotherapeutic drug currently used in the treatment of colorectal adenocarcinoma. Individuals carrying at least 1 copy of no function DPYD variant may not be able to metabolize 5-FU at normal rates and are at risk of life threatening toxicity such as neurotoxicity and bone marrow suppression. Associations between 5-FU treatment outcomes and germ line polymorphisms in DPYD ( ${ }^{\star} 9 \mathrm{~A},{ }^{\star} 9 \mathrm{~B},{ }^{\star} 4,{ }^{\star} 7$, $\left.{ }^{\star} 13,{ }^{\star} 2 \mathrm{~A}, \mathrm{D} 2846 \mathrm{~A}>\mathrm{T}, \mathrm{D} 1129-5923 \mathrm{C}>\mathrm{G}, \mathrm{D} 496 \mathrm{~A}>\mathrm{G}\right)$ were analyzed. The sequence variations of the DPYD gene with no functional consequences on enzymatic activity were detected to be $48,2 \%$ among 110 5-FU treated patient with colorectal adenocarcinoma. Among 110 patients 5 patients were poor metabolizers and 19 (17,3\%) were intermediate metabolizers for 5-FU whose clinicians were recommended to select an alternative drug. Whereas 32 patients were recommended to reduce dose by $25-50 \%$. The prevalence of DPD deficiency in Turkish study population was detected to be $4,5 \%$ in agreement with the published literature. In conclusion clinicians should be strongly encouraged to consider testing for DPD poor metabolizer variants as a rational pre-treatment screening for patients candidate to a fluoropyrimidine-based regimens, in order to prevent toxicities and personalized treatments.

Acknowledgement: This study was supported by Istanbul Development Agency, Project No. TR10/16/YNY/0030.

\title{
Pharmacogenetics as the basis of individualized therapy decisions
}

\author{
A Jetter \\ Department of Clinical Pharmacology and Toxicology, University Hospital Zurich and University of Zurich
}

In clinical routine patient care, pharmacogenetic information is only infrequently incorporated in therapy decisions. Pharmacogenetics may be used either before therapy initiation to find the right dose and the right drug, or thereafter, e.g. to explain side effects. Two pharmacogenetic testing approaches have been followed: A "holistic" approach where patients are screened for (the most relevant) pharmacogenetic variants before therapy initiation, or a more specific approach, where only in the case of a (currently given or planned) specific drug therapy the particular pharmacogenetics for this therapy are investigated. In Switzerland, only the latter approach is reimbursed by the health insurers under certain conditions. For a number of drug-gene pairs, sufficient scientific information is available to adjust therapy to pharmacogenetics. Examples are the selection and dose adjustments of anticancer chemotherapies in patients with defined variants in the genes for dihydropyrimidine dehydrogenase (DPYD) and UDP-glucuronoyl transferase UGT1A1, responsible for bioinactivation of 5-fluorouracil and irinotecan, respectively. These approaches are supported by scientific evidence and guidelines from pharmacogenetics consortia. In other cases, the situation is not as clear. For example in women who take in tamoxifen as prevention for relapse of breast cancer, there has been much debate about the association between CYP2D6 genotype and the risk of relapse. Since not only CYP2D6 genotype, but also CYP2C19 genotype and potential drug-drug interactions which lead to a decreased CYP2D6 (and / or CYP2C19) activity have to be taken into account, an individualized, patient-tailored approach seems to be more appropriate. This approach should include genotyping, interaction assessment and other factors of influence in the personalized therapy recommendation. In a number of other examples it is likewise important to first identify the underlying question and its therapeutic relevance before carrying out a pharmacogenetic test. Additional attention should be paid to the question which, and how, recommendations can be given after the pharmacogenetics tests have been carried out. In conclusion, it becomes clear that pharmacogenetics should be used more frequently in clinical routine, particularly in cases where good evidence and guidelines exist, because pharmacogenetics add insight in yet unidentified factors of influence on the individual drug therapy. However, recommendations will only be adequate if nongenetic factors of influence (like interactions, renal function, etc.) are also taken into account.

\section{Session IX - WHAT CAN WE LEARN FROM ELECTRONIC HEALTH RECORDS?}

\section{Using Big Data to interpret genomes to accelerate drug discovery}

\author{
Rong Chen \\ Icahn School of Medicine, Mount Sinaï, United States \\ No abstract available
}

\section{Electronic health records linked to genetics: role of national phenotyping efforts in the United Kingdom as a step towards an international phenome sequence project}

\author{
Harry Hemingway \\ UCLH BRC, University College London, London, UNITED KINGDOM
}

Backgound: Large-scale enquiry of longitudinal electronic health records (EHR) linked to genomic and 'omic' resources offers opportunities: (a) to pursue discovery science with well-established designs, including: GWAS, PheWAS, drug target validation, human knockouts; (b) to improve care through preventive genomics; and (c) for new kinds of scientific enquiry based on an agnostic understanding of longitudinal 
human phenome sequences. New EHR linkages in UK Biobank, the 100,000 genomes project among others span multiple EHR sources. As these data become available, there is a need to better understand their use in phenotyping.

Objectives: To review national efforts to define, validate and discover replicable, reusable phenotypes using EHR. To discuss the need to align phenotyping efforts across countries to enable international collaboration.

Design: The CALIBER platform (www.ucl.ac.uk/health-informatics-caliber) demonstrates phenotyping algorithms utilize five ontologies (Read (for primary care EHR), ICD-9, ICD-10, OPCS-4, BNF), covering diagnosis codes, symptoms, procedure codes, medications and continuous measurements.

Results: CALIBER has developed phenotyping algorithms for 72 diseases and risk factors and made them available through an open-access Data Portal. It has also developed and released a set of open source tools. We discuss the role of Health Data Research UK, the new UK data science institute and the international context. Different approaches to developing EHR phenotypes, including: one at a time vs high throughput phenotyping, clinical Boolean rules based, vs regression based, vs machine learning; simple vs complex phenotypes and the role of real time phenotyping in clinical decision making. We discuss different approaches to validating EHR phenotypes, including epidemiological (1), cross-source (2), genomic, prognostic, PPV 'case note review' and text. Lastly, we discuss approaches to discovering EHR sub-phenotypes and endotypes with machine learning and genomic validation.

Conclusions: In the era of precision medicine, robust approaches to creating, validating and sharing EHR-derived phenotypes are critical to enable cross-source analyses of thousands of simple and complex traits in millions of individuals. The CALIBER approach provides a transparent methodology for transforming raw EHR to reproducible phenotypes for use in such studies.

References

1. Rapsomaniki E. et al., Lancet, 2014, doi: 10.1016/S0140-6736(14)60685-1

2. Herrett E. et al., BMJ, 2012, doi: 10.1136/bmj.f2350

Primary health data in a cohort of over 20,000 people in East London

Richard Trembath

King's College, London, United Kingdom

No abstract available

\title{
Linking genetic data to risk of recurrent cardiovascular events through hospital records
}

\author{
Panagiotis Deloukas
}

Queen Mary University London, London, United Kingdom

No abstract available

\section{CONCLUSIONS \& PERSPECTIVES REMARKS}

by Sofia Siest, Nancy, France and Urs Meyer, Basel, Switzerland 


\section{POSTERS}

\section{Group 1 - "-Omics" biomarkers}

The beneficiary effects of $n-3$ polyunsaturated fatty acids on non-alcoholic steatohepatitis in mice Libor Vitek

Changes in plasma metabolomic profile during acute myeloid leukemia treatment

Alzbeta Hlavackova ${ }^{1}$, Leona Chrastinova ${ }^{1}$, Jan Vydra ${ }^{2}$, Jiri Suttnar ${ }^{1}$, Jan E. Dyr ${ }^{1}$

Genetic determinants of lipidomic variation of a new class of insulin-sensitizing agents - fatty acid esters of hydroxy fatty acids

Michal Pravenec ${ }^{1}$, Ondrej Kuda ${ }^{1}$, Kreuchwig Franziska², Thierry Durand ${ }^{3}$, Norbert Hubner ${ }^{2}$, Aron Fisher ${ }^{4}$, Jan Kopecky ${ }^{1}$,

In vivo effects of HIPEC in gastric cancer specimen

Archil Aladashvili ${ }^{1}$, Roland Croner ${ }^{2}$, Tengiz Ismailovi ${ }^{3}$, Simon Kitiashvili ${ }^{4}$, Vakhtang Begiashvili ${ }^{4}$, Vakhtang Tsabadze ${ }^{4}$

Gene score as predictor of hypertriglyceridemia in Czech population

Jaroslav A. Hubacek ${ }^{1}$, Vera Adamkova ${ }^{2}$, Michal Vrablik ${ }^{3}$, Lucie Schwarzova ${ }^{4}$, Richard Ceska ${ }^{4}$

Jaroslav Hubacek ${ }^{1}$, Dana Dlouha ${ }^{1}$, Lukas Tichy², Lenka Fajkusova ${ }^{2}$, Tomas Freiberger ${ }^{3}$

Cardiovascular mortality is not associated with plasma bilirubin concentration or UGT1A1 polymorphism

Jaroslav Hubacek ${ }^{1}$, Libor Vitek ${ }^{2}$, Andrzej Pajak ${ }^{3}$, Denes Stefler ${ }^{4}$, Martin Bobak ${ }^{4}$

Alterations in the Gene Expression of Drug and Arachidonic Acid-Metabolizing CYP450 in the Livers of Controlled and Uncontrolled InsulinDependent Diabetic Mice

Yazun Jarrar

Sphingosine-kinase 1 and epithelio-mesenchymal transition in lung adenocarcinomas

Pierre Fouret

Aqueous Extract of Selected Wild Vegetables Reduce Blood Pressure and Improve Cardiac Functions in Isoproterenol-Induced Myocardial Infarction Male Rats

eA26

Kehinde Bolarinwa KA

Relationship between metabolic syndrome and dry eye symptoms

eA27

Malgorzata Mrugacz, Anna Bryl

Monitoring of myelodysplastic syndrome progression based on lectin array and analysis of protein interactions

Leona Chrastinova ${ }^{1}$, Ondrej Pastva ${ }^{1}$, Marketa Bockova ${ }^{2}$, Jaromir Novak ${ }^{1}$, Jana Stikarova ${ }^{1}$, Alzbeta Hlavackova ${ }^{1}$, Jiri Suttnar ${ }^{1}$, Roman Kotlin ${ }^{1}$, Jiri Homola ${ }^{2}$, Jaroslav Cermak ${ }^{1}$, Jan Dyr ${ }^{1}$,

Association of beta-adrenergic receptors polymorphisms with Body Mass Index in a Southeastern European Caucasian population eA28 Aikaterini Karathanasopoulou, Giwrgos Vlachos, Athanasios Mouchtaris-Michailidis, Maria Papasavva, Angeliki Andrianopoulou, MarthaSpyridoula Katsarou, Maria Lagiou, Nikolaos Drakoulis

Tryptophan metabolites and oxidative stress in patients with cardiovascular disease or stroke

Jiri Suttnar ${ }^{1}$, Alzbeta Hlavackova'1, Martin Hajsl², Karolina Broulikova², Martin Sramek³, Martin Maly², Jan Dyr ${ }^{1}$

Phthalates exposure influence on obesity development and glucose homeostasis

Natasa Milosevic ${ }^{1}$, Maja Milanovic ${ }^{1}$, Kristina Stepanovic ${ }^{1}$, Stefan Stojanoski ${ }^{1}$, Dragana Bosic Zivanovic ${ }^{2}$, Natasa Milic ${ }^{1}$, Milica Medic Stojanoska ${ }^{1}$

Inflammation Related TNF- $\alpha$, IL-6 and CRP Gene Polymorphisms in a Southeastern European Caucasian Population Sample and Comparison with Other Populations

Maria Papasavva, Evgenia Georgiadi, Alexandra Petraina, Martha-Spyridoula Katsarou, Maria Lagiou, Panagiota Stamou, Thodoris Kalogridis, Nikolaos Drakoulis 
Population Based Analysis of GSTP1 Gene Polymorphisms Distribution In A Southeastern European Caucasian Population Sample And Comparison With General European Population Frequency Distributions

Konstantina Doulou, Evangelia Alexiou, Martha Katsarou, Rozana Latsi, Maria Papasavva, Maria Lagiou, Nikolaos Drakoulis

Cardiovascular disease biomarker discovery using network biology

Karla V. Allebrandt ${ }^{1}$, Fancesca Frau ${ }^{1}$, Sibylle Hess² ${ }^{2}$ Hertzel Gerstein ${ }^{3}$, Daniel Crowther ${ }^{1}$, Guillaume Pare

Systems biology analysis of interaction and regulation networks in high throughput RNAi screenings

Ghislain Bidaut ${ }^{1}$, Quentin Da Costa ${ }^{1}$, Claire Rioualen ${ }^{2}$, Bernard Chetrit ${ }^{1}$, Emmanuelle Charafe-Jauffret ${ }^{1}$, Christophe Ginestier ${ }^{1}$

SiMS score in obese children, adolescents and youth increases with age

Vesna Dimitrijevic-Sreckovic ${ }^{1}$, Hristina Janeski², Branko Sreckovic ${ }^{3}$, Mirjana Popadic ${ }^{4}$, Ivan Soldatovic ${ }^{5}$

Confounding factors of metabolic syndrome-confirmed by siMS score and siMS risk score

Branko Sreckovic ${ }^{1}$, Vesna Dimitrijevic- Sreckovic ${ }^{2}$, Hristina Janeski ${ }^{3}$ Emina Colak ${ }^{4}$, Nenad Janeski ${ }^{5}$, Ivan Soldatovic ${ }^{6}$

Framingham coronary heart disease risk does not correlate with coronary calcium burden in patients with essential thrombocytosis Matjaž VRTOVEC

L-2-hydroxyglutaric aciduria-case report

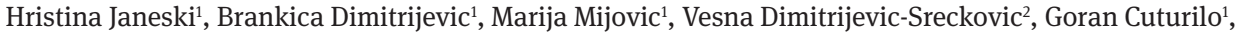

Obesity, metabolic syndrome and prediabetes are associated with increased cancer risk

Vesna Dimitrijevic-Sreckovic ${ }^{1}$, Milica Vujovic ${ }^{2}$, Hristina Janeski ${ }^{3}$, Branko Sreckovic ${ }^{4}$, Nevena Pajovic ${ }^{2}$, Sasa Ilic ${ }^{2}$, Ivan Soldatovic ${ }^{5}$

Molecular genetic diagnosis of monogenic forms of diabetes in Russia

Affaf ADDA ${ }^{1}$, Mohamed CHEKKAL 2 , Nazim BENNAOUM², Mohammed HAMMADI ${ }^{2}$

Uncovering Clinically Relevant Mutations in Membrane Transporters by Genetic Analysis Linked to the Determination of Erythrocyte Membrane Protein Expression

Balazs Sarkadi

Association of the genotype and metabolic profile of athletes in the formation of adaptations to physical activity 
The VEGF Consortium

eA39

MG Stathopoulou ${ }^{1}$, T Xie ${ }^{1}$, D Ruggiero², J Chatelin ${ }^{1}$, M Rancier ${ }^{1}$, G Weryha ${ }^{1}$, MJ Kurth ${ }^{3}$, AA Aldasoro Arguinano ${ }^{1}$, V Gorenjak ${ }^{1}$, AM Petrelis $^{1}$, G Dagher ${ }^{4}$, G Dedoussis ${ }^{5}$, P Deloukas ${ }^{6}$, L Lamont ${ }^{3}$, J Marc ${ }^{7}$, M Simmaco $^{8}$, RHN Van Schaik ${ }^{9}$, F Innocenti ${ }^{10}$, JL Merlin ${ }^{11}$, J Schneider ${ }^{12}$, BZ Alizadeh ${ }^{13}$, M Ciullo ${ }^{2}$, S Seshadri ${ }^{14}$, S Visvikis-Siest ${ }^{1}$; VEGF Consortium.

TREM-1 variants associations with TREM-1 protein and mRNA levels and adhesion molecules

AA Aldasoro Arguinano ${ }^{1}$, S Dadé ${ }^{1}$, MG Stathopoulou ${ }^{1}$, M Derive $^{2}$, NC Ndiaye ${ }^{1}$, T Xie $^{1}$, C Masson $^{1}$, S Gibot $^{3}$, S Visvikis-Siest ${ }^{1,4}$

Associations of polymorphisms in the region of the TREM2 gene with TNF- $\alpha$ levels and IMT-F

V Gorenjak ${ }^{1}$, AA Aldasoro Arguinano ${ }^{1}$, S Dadé 1 , MG Stathopoulou ${ }^{1}$, D Vance ${ }^{2}$, C Masson ${ }^{1}$, S Visvikis-Siest ${ }^{1,3}$

A case of Constitutional mismatch Repair Deficiency Syndrome caused by PMS1

Reem Salah ${ }^{1}$, Rayan Seifeldin ${ }^{1}$, Aymen Hussein ${ }^{1}$, Imad Fadl-Almula², Muntaser E Ibrahim ${ }^{1}$

Analysis of CFTR by next-generation sequencing in patients with cystic fibrosis from West

Olga Vladimirovna Romanova ${ }^{1}$, Tatyana Eduardovna Ivashchenk², Oleg Sergeevich Glotov ${ }^{1}$, Michael Andreevich Fedyakov ${ }^{1}$, Yuliya Almazovna Nasyhova ${ }^{2}$, Anna Valer'yanovna Gorbunova ${ }^{3}$, Yury Aleksandrovich Barbitov ${ }^{4}$, M Donnikov $^{5}$, L Kolbasin ${ }^{5}$, Andrei Mikhaylovich Sarana ${ }^{1}$, Sergei Grigorievich Scherbak ${ }^{1}$, Vladislav Sergeevich Baranov ${ }^{2}$

\section{POSTERS}

\section{Group 2 - PHARMACOGENOMICS}

Study of CYP2D6 genotype-phenotype in a deprescription plan with prescription opioid dependence patients

eA41

Ana María Peiró

The DPYD for precision Medicine in Breast Cancer chemotherapy among Sudanese

Fatima Hamadto, Maha Othman, Ameera Adam and Muntaser Ibrahim

The perils and possibilities of p53-based personalized cancer therapy

eA42

Z H Siddik ${ }^{1}$, Guangam He, Xiaolei Xie

The Influence of CYP2D6 1846G > A Polymorphism on the Efficacy and Safety of Fluvoxamine in Patients with Depressive Disorder and Comorbid Alcohol Use Disorder

Zastrozhin Mikhail ${ }^{1}$, Grishina Elena ${ }^{2}$, Savchenko Ludmila ${ }^{3}$, Bryun Evgeny ${ }^{4}$, Sychev Dmitry ${ }^{5}$

The interest of Therapeutic Drug Monitoring of Infliximab

Toumi Houari

EHU Oran Department Pharmacovigilance, Ora, ALGERIA

COQ2 polymorphisms are not associated with increased risk of statin induced myalgia/myopathy in Czech population

eA44

Jaroslav Hubacek ${ }^{1}$, Vera Adamkova², Lukas Zlatohlavek ${ }^{3}$, Lenka Steiner-Mrazova', Michal Vrablik ${ }^{3}$

Synthetic Switch-based Baculovirus for Transgene Expression Control and Selective Killing of Hepatocellular Carcinoma Cells

Yu Cheng Andy,

Pharmacogenetic study of clopidogrel in Caribbean Hispanics

J Duconge ${ }^{1}$, DF Hernandez-Suarez ${ }^{1}$, KI Claudio-Campos ${ }^{1}$, SA Scott², MI Tomey ${ }^{2}$, MR Botton², PA Villablanca ${ }^{3}$, K Melin ${ }^{1}$, A Lopez-Candales ${ }^{1}$, JY Renta ${ }^{1}$

The highest joint prevalence of CYP2C9*2 and VKORC1 variants responsible for warfarin sensitivity in the Croatian Roma compared to 20 populations worldwide

Tatjana Škaric-Juric, Branka Janicijevic, Nina Smolej Narancic, Matea Zajc Petranovic, Željka Tomas, Marijana Pericic Salihovic

Distinctiveness of the Roma population in pharmacogenetic "core list" markers within the worldwide populations 
Long term metabolic improvements in morbid obese diabetic and non-diabetic patients after Roux-en-Y Gastric Bypass (RYGB): a stable isotope tracer study eA46 Chiara Barbieri ${ }^{1}$, Chiara Barbieri² ${ }^{2}$ Fabrizia Carli ${ }^{1}$, Melania Gaggini ${ }^{1}$, Brenno Dominguez Brenno Dominguez ${ }^{4}$, Marianna Palumbo ${ }^{4}$, Ele Ferrannini $^{1}$, Stefania Camastra ${ }^{4}$, Amalia Gastaldelli ${ }^{1}$

Comparison study of CYP2C9 (rs1799853, rs1057910), CYP2C19 (rs4244285), CYP1A2 (rs762551), CYP11B2 (rs1799998), CYP17 (rs619824) frequency distribution between Southeastern European Caucasians and other populations

Maria Papasavva, Danae Theodoridou, George Diles, Martha-Spyridoula Katsarou, Maria Lagiou, Thodoris Kalogridis, Nikolaos Drakoulis

Identification of genetic changes relevant to radiation response using targeted next-generation DNA sequencing Eui Kyu Chie, Seung Hyuck Jeon, Yi-Jun Kim, Joo Ho Lee

Correlation of Polymorphisms in SCN1A Gene with Diagnosis and Effective Therapy of Focal Impaired Awareness Seizures Danae Tsikrika ${ }^{1}$, Anastasia Verentzioti ${ }^{2}$, Panagiotis Patrikelis ${ }^{2}$, Nikolaos Drakoulis1, Eleni Kokkiou ${ }^{1}$, Martha Katsarou ${ }^{1}$, Anna Siatouni ${ }^{2}$, Stylianos Gatzonis², Michael Koutsilieris ${ }^{3}$, Ioannis Sakkas³, Athanasia Alexoudi²

Association of CNS Genes OPRM1, DRD2 and BDNF Polymorphisms with Nicotine Addiction in a Southeastern European Population eA48 Anastasia Zoulia, Stavroula Nioma, Konstantinos Sarantidis, Eleni Kokkiou, Martha Katsarou, Maria Lagiou, Thodoris Kalogridis, Nikolaos Drakoulis

Pharmacogenetics of clopidogrel in coronary artery disease patients: Results from Turkish from clinical practice Cevik Mehtap ${ }^{1}$, Susleyici Belgin ${ }^{1}$, Ciftci Cavlan², Karaalp Atila ${ }^{3}$, Yurdakul Selen², Canbolat Ismail Polat², Gezer Elifcan¹, Bezci Kivanc ${ }^{1}$, Cagatay Penbe $^{4}$, Schaik Ron ${ }^{5}$

The impact of CYP2D6 mediated drug-drug interaction: a systematic review on a combination of metoprolol and paroxetine/fluoxetine eA49 Muh. Akbar Bahar'1, Jasper Kamp¹, Sander D Borgsteede ${ }^{2}$, Eelko Hak¹, Bob Wilffert ${ }^{1}$

Mutated cyclin dependent kinase 4 and Retinoblastoma 1 may promote chemo-resistance to palbociclib in ovarian cancer eA50 Said El Shamieh ${ }^{1}$, Fatima Saleh ${ }^{1}$, Fadi Farhat ${ }^{2}$

Pharmacogenomics and Phenotypic Alterations as Predictive Biomarkers of Fluoropyrimidine-based Chemotherapy Toxicity eA50 Roberto Michela, Arrivi Giulia, Romiti Adriana, Lionetto Luana, Botticelli Andrea, Di Pietro Francesca Romana, Mazzuca Federica, Simmaco Maurizio, Marchetti Paolo

Implementation of a pharmacogenetic panel test service and its potential clinical impact on medication costs in Finland Juho Heliste ${ }^{1}$, Klaus Elenius ${ }^{1}$, Varpu Elenius ${ }^{2}$

Real-world outcomes in patients with metastatic melanoma treated with anti-PD1 agent nivolumab

Ekaterina Shakhray ${ }^{1}$, Igor Samoylenko¹, Galina Kharkevich¹, Natalia Petenko¹, Yaroslav Zhulikov², Lev Demidov ${ }^{1}$

Association between MTHFR C677T gene polymorphisms and cardiovascular adverse events in patients with metastatic colorectal cancer treated with 5-fluorouracil based chemotherapy

Arrivi Giulia, Roberto Michela, Di Pietro Francesca Romana, D’Antonio Chiara, Milano Annalisa, Macrini Serena, Marchetti Paolo, Mazzuca Federica

Rare variants in CYP2C9 and CYP3A5 detected in patients with familial hypercholesterolemia with statin-related adverse events Carolina Dagli-Hernandez¹, Jéssica Bassani Borges ${ }^{1}$, Bruna Los¹, Andre Arpad Faludi², Rodrigo Marques Gonçalves², Mario Hiroyuki Hirata ${ }^{1}$, Rosario Dominguez Crespo Hirata ${ }^{1}$

Genetic association study to identify biomarkers suitable for individualized dug therapy for diabetic and hypertensive patients in South Africa

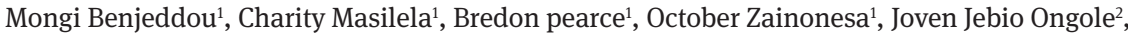

Oral Alpha-Cyclodextrins for the Disease-Modifying Treatment and the Prevention of Age-related diseases (including Cancer, Atherosclerosis, Alzheimer's / Parkinson's disease, and Multiple Sclerosis) 


\title{
POSTERS
}

\section{Group 1 - "-Omics" biomarkers}

\section{The beneficiary effects of $n-3$ polyunsaturated fatty acids on non-alcoholic steatohepatitis in mice}

\author{
Libor Vitek \\ 1st Faculty of Medicine, Charles University, Prague, Czech Republic
}

Introduction. Non-alcoholic fatty liver disease (NAFLD) and non-alcoholic steatohepatitis (NASH) represent the most common chronic liver diseases in western countries with a substantial risk of liver cirrhosis and high cardiovascular morbidity and mortality. The aim of the study was to determine the effects of administration of $n-3$ polyunsaturated fatty acids (PUFA) in the high fat, methionine-choline deficient (MCD) mouse model of NASH.

Methods. Male C57BL/6 mice were fed for 6 weeks either with MCD or with chow, and supplemented with n-3 PUFA (docosahexaenoic acid, $2.4 \mathrm{mg} / \mathrm{kg}$; and eicosapentaenoic acid, $3.2 \mathrm{mg} / \mathrm{kg}$ ). Analyses of serum biochemistry, pro-inflammatory cytokines, liver histology and hepatic mRNA transcriptoms were performed in the studied groups. Lipidomic analyses were performed by using UHPLC-HRMS analyses. Large chromatographic and spectral data sets generated by metabolic fingerprinting were used to assess the differences between groups. BeadChip analysis was used to profile and identify the differences in liver mRNA transcriptomes.

Results. n-3PUFA administration ameliorated histopathological picture of NASH in MCD mice, decreased cholesterol levels, aminotransferase activities, as well as pro-inflammatory cytokine profile ( $\mathrm{p}<0.01$ for all comparisons). In addition, $\mathrm{n}-3$ PUFA treatment led to lower serum concentrations of saturated and monounsaturated free fatty acids and higher serum concentrations of n-3 PUFA, and also the content of liver triacylglycerols $(\mathrm{p}<0.01)$. The analysis of the liver lipidomics data separated dietary interventions into distinct groups according the presence of $\mathrm{n}-3$ PUFA in the diet demonstrating big influence of n-3 PUFA on liver lipid metabolism. BeadChip analysis revealed significant differences in liver mRNA transcriptomes between n-3 PUFA-treated MCD animals and controls, with $>4700$ gene transcripts significantly changed ( $p<0.05$ ). Among others, expressions of genes affecting cholesterol and fatty acid biosynthesis pathways, as well as those involved in inflammatory pathways were the most important protectively modulated by n-3 PUFA.

Conclusion. In this mouse model of NASH, n-3 PUFA have favorable effects on histopathological features of NASH, serum markers of liver damage, serum as well as hepatic metabolomic profile.

Keywords: NASH, NAFLD, PUFA, polyunsaturated fatty acids

\section{Changes in plasma metabolomic profile during acute myeloid leukemia treatment}

\author{
Alzbeta Hlavackova ${ }^{1}$, Leona Chrastinova ${ }^{1}$, Jan Vydra², Jiri Suttnar ${ }^{1}$, Jan E. Dyr ${ }^{1}$ \\ 1. Department of Biochemistry, Institute of Hematology and Blood Transfusion, Prague, Czech Republic \\ 2. Clinical division, Institute of Hematology and Blood Transfusion, Prague, Czech Republic
}

Background: Acute myeloid leukemia (AML) represents an aggressive bone marrow malignancy. It is the most common malignant myeloid disease in adults. AML is described as a heterogeneous clonal disorder of haemopoietic progenitor cells. The cells show characteristic changes in metabolism, including increased uptake of glucose and glutamine and synthesis of essential biomolecules, that support tumor cells growth and survival [1]. Although metabolic abnormalities in cancer cells were recognized several decades ago, understanding of individual AML metabolism pathways represents an important therapeutic target.

Objective: By using targeted metabolomic analysis we studied the changes in plasma concentration of 19 selected intermediates of glycolysis, tricarboxylic acid cycle, and amino acid metabolisms during AML patient's diagnosis, treatment, and remission.

Design: In this study, we examined plasma samples from 23 AML patients in three different periods - in diagnosis, after treatment, and in remission before transplantation. As a control the same number of healthy donors was used. A quantitative metabolomics profiling of plasma samples was provided by tandem mass spectrometry (LC-MS/MS).

Results: Using targeted metabolomic analysis we found significant differences in plasma concentration of 16 metabolites between all AML patients and healthy donors. Furthermore, 14 metabolites were significantly elevated in samples of AML patients at the time of in diagnosis compared to healthy donors, 9 metabolites were significantly elevated in samples of AML patients after treatment compared to healthy donors, and 11 metabolites were significantly elevated in samples of AML patients in diagnosis compared to their state in remission.

Conclusions: This study reveales that knowledge of changes in various intermediate concentrations of selected metabolic pathways during the diagnosis, course, and treatment of AML could lead to the understanding of the occurrence, progression, and prognosis of this serious disorder.

Acknowledgements: This work was supported by the European Regional Development Fund and the state budget of the Czech Republic (project AIIHHP: CZ.02.1.01/0.0/0.0/16_025/0007428, OP RDE, Ministry of Education, Youth and Sports), by the project of the Ministry of Health, Czech Republic, 00023736, by Grant from the Academy of Sciences, Czech Republic, P205/12/G118, and by ERDF OPPK CZ.2.16/3.1.00/24001. References: Benjamin DI, Cravatt BF, Nomura DK. Cell Metab. 2012; 16(5): 565-577. 


\section{Genetic determinants of lipidomic variation of a new class of insulin-sensitizing agents - fatty acid esters of hydroxy fatty acids}

Michal Pravenec ${ }^{1}$, Ondrej Kuda ${ }^{1}$, Kreuchwig Franziska², Thierry Durand ${ }^{3}$, Norbert Hubner ${ }^{2}$, Aron Fisher ${ }^{4}$, Jan Kopecky ${ }^{1}$

1. Institute of Physiology of the Czech Academy of Sciences, Prague, Czech Republic

2. Max-Delbruck-Center for Molecular Medicine, Berlin, Germany

3. Institut des Biomolecules Max Mousseron (IBMM), UMR 5247, CNRS, Universite Montpellier, Montpellier, France

4. Institute for Environmental Medicine of the Department of Physiology, University of Pennsylvania, Philadelphia, United States

Background: Fatty acid esters of hydroxy fatty acids (FAHFAs) are lipid mediators with promising anti-diabetic and anti-inflammatory properties that are formed in white adipose tissue (WAT) via de novo lipogenesis. Levels of one of the FAHFA family members, palmitic acid hydroxystearic acid (PAHSA), are markedly lower in serum and WAT of insulin-resistant humans, however, their biosynthetic enzymes are unknown. Design: Using a combination of lipidomics in WAT, QTL mapping and correlation analyses in rat BXH/HXB recombinant inbred strains derived from SHR (spontaneously hypertensive rat) and BN (Brown Norway) progenitors, and response to oxidative stress in murine models, we elucidated the potential pathway of biosynthesis of several FAHFAs. Results: Comprehensive lipidomic analysis of WAT samples identified $\sim 160$ regioisomers documenting the complexity of this lipid class. The linkage analysis highlighted several members of Nuclear factor, erythroid 2-like 2 (Nrf2)-mediated antioxidant defense system (Prdx6, Mgst1, Mgst3, Gpx7), lipid-handling proteins (Cd36, Scd6, Acnat1, Acnat2, Baat) and family of Flavin Containing Monooxygenase (Fmo) as the positional candidate genes. Transgenic expression of Nrf2 and deletion of Prdx6 genes resulted in reduction of palmitic acid ester of 9-PAHSA and 11-PAHSA levels, while oxidative stress induced by an inhibitor of glutathione synthesis increased PAHSA levels nonspecifically.

Conclusions: Our results indicate that the synthesis of FAHFAs via carbohydrate-responsive element-binding protein (ChREBP)-driven de novo lipogenesis depends on the adaptive antioxidant system and suggest that FAHFAs may link activity of this system with insulin sensitivity in peripheral tissues. These findings of basic research may be important clinically because there is a major unmet need for safe insulinsensitizing agents for treatment of patients with type 2 diabetes since the only available insulin-sensitizing drugs, thiazolidinediones, have adverse effects. Acknowledgements: This research was supported by grant 16-04859S from the Czech Science Foundation.

\section{In vivo effects of HIPEC in gastric cancer specimen}

Archil Aladashvilii', Roland Croner ${ }^{2}$, Tengiz Ismailovi ${ }^{3}$, Simon Kitiashvili ${ }^{4}$, Vakhtang Begiashvili ${ }^{4}$, Vakhtang Tsabadze ${ }^{4}$ 1. Surgery, TSMU, Tbilisi, Georgia

2. Surgery, Uniklinik, Magdeburg, Germany

3. Surgery, NCC, Tbilisi, Georgia

4. Lab, TSMU, Tbilisi, Georgia

Background: The currently most acceptable treatment option for advanced GC (AGC) is surgery, systemic chemo-radio therapies with however generally very unsatisfying results and this led to a resurgence of interest in regional therapies like hyperthermic intraperitoneal chemotherapy (HIPEC). as a prophylactic measure to prevent peritoneal recurrence after gastrectomy in high risk patients. We aimed to study the in vivo effects of HIPEC after radical curative surgery in gastric cancer specimen.

Methods: We investigate tumor and non-neoplastic peritoneal tissue samples from 110 patients undergoing parcial or total gastrectomy, D2 lymph dissection and prophylactic HIPEC for AGC, We examined conventional morphology and immunohistochemical analysis for markers of early necrosis (extracellular High mobility group box 1 protein - HMGB1). Findings were correlated with clinicopathologic features of disease free period, short-term and 3-year survival.

Results: Microscopic findings in vivo investigation of HIPEC included extensive submesothelial inflammatory infiltrate, endothelial activation, mesothelial karyolysis and surface fibrin deposition. Endothelial activation in submesothelial vessels exhibited high specificity for samples obtained following HIPEC relative to samples obtained prior to HIPEC. No significant increase was seen in tumor staining for markers with HIPEC. Submesothelial HMGB1 staining was associated with increased progression-free survival on gastric cancer specimen.

Conclusions: In vivo and Histology effects of HIPEC in gastric cancer specimen are defined and provide evidence that DNA damage and early steps of necrosis are underway in mesothelial tissues at the conclusion of the procedure. Further research will be necessary to investigate the impact of these findings on long-term oncologic outcome and survival, and may provide insight into the downstream effects of HIPEC that could facilitate refinement of loco-regional therapeutic regimens to avoid gastric cancer carcinomatosis.

\section{Gene score as predictor of hypertriglyceridemia in Czech population.}

Jaroslav A. Hubacek ${ }^{1}$, Vera Adamkova ${ }^{2}$, Michal Vrablik³ ${ }^{3}$ Lucie Schwarzova ${ }^{4}$, Richard Ceska ${ }^{4}$

1. Department of experimental medicine, Institute for clinical and experimental medicine, Prague, Czech Republic

2. Institute for experimental medicine, Prague, Czech Republic

3. Ist MF UK, Prague, Czech Republic

4. 1st MF UK, Prague, Czech Republic 
Background: Hypertriglyceridemia (HTG) is a common lipid disorder. It is supposed, that really high plasma levels of triglycerides (over $10 \mathrm{mmol} / \mathrm{L}$ ) have strong genetic background. Rare mutations explain some cases, but in majority of the patients, the disease seems to be polygenic. Accumulation of risky alleles can nonlinearly increase the risk of HTG.

Objective: To analyze the gene score distinguishing potentially between patients with high TG values and normotriglyceridemic controls. Design: We have analysed common polymorphisms within the APOA5, FRMD5, GCKR, CAPN3, TRIB1, MLXIPL, LPL, LIPC, TYW1B, ANGPLT3, COBLL1, CILP2, NAT2D, PLTP, FADS1-2-3, TIMD4, CETP and GALNT2 genes in 203 patients with plasma TG over 10 mmol/L and 505 treatment naïve controls with plasma TG repeatedly below $1.8 \mathrm{mmol} / \mathrm{L}$.

Results: Except for two exceptions (FADS1-2-3, GALNT2 genes), we have found significant risk (all $\mathrm{P}<0.01$ and ORs over 1.8) of hypertriglyceridemia development associated with risky alleles of the above mentioned SNPs. The extreme association has been observed in the case of the APOA5 gene (GG vs. T allele carriers - OR 15.16, 95\%CI 6.40 - 36.04; P < 0.000001). The mean of risky alleles/person was $12.2 \pm 2.2$ in HTG group vs. $9.9 \pm 1.9$ in controls $(\mathrm{P}<0.0001)$. Unweighted gene score (using SNPs within the five most significant genes - APOA5, NAT2, LIPC, LPL a TRIB1) shows, that carriers of at least 5 risky alleles exhibit 15-times higher risk to develop HTG than carriers of max 3 risky alleles (OR, 95\%CI 14.9, 8.9 - 25.2; P $<0.00000001)$.

Conclusion: Our results clearly confirm that the common SNPs are strong predictors of the HTG development.

Acknowledgements: Supported by Ministry of Health of the Czech Republic, grant nr. 15-28876A. All rights reserved.

Keywords: hypertriglyceridemia, polymorphism, gene score

\section{Common polymorphisms are unlikely to play a major role in pseudo - FH development in Czech population}

Jaroslav Hubacek ${ }^{1}$, Dana Dlouha ${ }^{1}$, Lukas Tichy², Lenka Fajkusova², Tomas Freiberger ${ }^{3}$

1. Department of experimental medicine, Institute for clinical and experimental medicine, Prague, Czech Republic

2. Centre of Molecular Biology and Gene Therapy, University Hospital Brno, Brno, Czech Republic

3. Molecular Genetics Laboratory, Centre for Cardiovascular Surgery and Transplantation, Brno, Czech Republic

Background: Familial hypercholesterolemia (FH) is a serious disease, leading (if not treated) to premature myocardial infarction. FH is caused predominantly by mutations within the LDL receptor and APOB genes. Recently, gene scores have been defined, suggesting the existence of "pseudo - FH", clinically indistinguishable from classical FH, but without one causal mutation. In our study, we have analyzed 17 LDL-cholesterol associated (in general population) SNPs in Czech patients with FH.

Objective: To analyze the role of common polymorphisms as a potential determinant of clinically, but not genetically, confirmed FH. Design: Polymorphisms within PCSK9, NYNRIN, ST3G, SORT1 (two SNPs), ABCG8, LDLR, APOB (two SNPs), SLC22, PPP1R3, MYLIP, HFE, CETP, CILP/PBX4, BRAP and HMGCoAR were genotyped using Taqmann technology on an AB 7300 RT PCR cycler or using PCR-RFLP in 298 FH patients without the causal mutation and in 296 patients with the LDL receptor mutation.

Results: Frequencies of the individual genotypes significantly differ between two analyzed groups only in the case of CILP/PBX4 - rs1699148 $(\mathrm{P}<0.005)$ and APOB - rs1367117 $(\mathrm{P}<0.005)$ genes. Genotype frequencies of other polymorphisms did not differ between the analysed groups. Conclusion: The results from our study suggest that common genetic variants will not play a major role in the "pseudo-FH" development in Czech population. Only two of seventeen analysed SNPs seem to be of clinical importance and to influence the development of the pseudo FH phenotype.

Acknowledgements: Supported by Ministry of Health of the Czech Republic, grant nr. 15-28277A. All rights reserved. Keywords: familial hypercholesterolemia, polymorphism, cholesterol

\section{Cardiovascular mortality is not associated with plasma bilirubin concentration or UGT1A1 polymorphism}

Jaroslav Hubacek ${ }^{1}$, Libor Vitek ${ }^{2}$, Andrzej Pajak ${ }^{3}$, Denes Stefler ${ }^{4}$, Martin Bobak ${ }^{4}$

1. Centre for experimental medicine, Institute for clinical and experimental medicine, Prague, Czech Republic

2. 1st faculty of medicine, Charles University, Prague, Czech Republic

3. DEPS, Jagiellonian University Medical College, Krakow, Poland

4. Department of Epidemiology and Public Health, UCL, London, United Kingdom

Introduction: Gilbert's syndrome is defined as a genetically determined (TA repeat in the bilirubin UDP-glucuronosyl transferase gene promoter, UGT1A1) disease characterized by mild hyperbilirubinemia in the absence of hepatic disease. The protective antioxidant role of bilirubin has recently been discussed in terms of atherosclerosis development.

Objective: To analyse the relationship of bilirubin and Gilbert's mutation/TA repeat polymorphism to cardiovascular mortality.

Design: The bilirubin concentration and TA repeat polymorphism within the UGT1A1 gene were analyzed in the part of the HAPIEE study (age 45-69 years, $N=7,688$ individuals), followed up for eight years. During this time, a total of 447 deaths were reported, of which 139 were cardiovascular. A further 1,423 individuals were selected from the cohort of survivors as a control for detailed analysis. In total, 1,870 individuals were examined. ANOVA and chi-sq. were used for statistical analysis.

Results: UGT1A1 (TA)7/7 homozygotes $(\mathrm{N}=267)$ had significantly $(\mathrm{P}<0.001)$ higher bilirubin levels $(20.3 \pm 7.7 \mu \mathrm{mol} / \mathrm{L})$ than carriers of at least one (TA) 6 allele $(\mathrm{N}=1603 ; 13.1 \pm 4.2 \mu \mathrm{mol} / \mathrm{L})$. Cardiovascular mortality was not significantly affected by either the bilirubin concentration or (TA)6/7 polymorphism. The results remained nonsignificant in all models used (dominant, co-dominant and recessive) even after adjustment for age, gender, education, smoking, alcohol intake, body mass index, basic lipid parameters and energy intake. 
Conclusion: Our results do not confirm that bilirubin concentration or (TA) repeat polymorphism within the UGT1A1 gene is associated with cardiovascular mortality in the general population.

Acknowledgements: Supported by Ministry of Health of the Czech Republic, grant nr. 15-28895A. All rights reserved.

Keywords: bilirubin, cardiovascular mortality, polymorphism, UGT1A1

\section{Alterations in the Gene Expression of Drug and Arachidonic Acid- Metabolizing CYP450 in the Livers of Controlled and Uncontrolled Insulin-Dependent Diabetic Mice}

\section{Yazun Jarrar}

Pharmacy, AlZaytoonah University, Amman, Jordan

Background: Diabetic patients have lower capacity to metabolize drugs in comparison to normal people. Therefore, the present study aimed to investigate the alterations in gene expression of drug and arachidonic acid metabolizing cytochrome p450s (cyp450s) in the livers of controlled (CDM) and uncontrolled (UDM) insulin-dependent diabetic mice.

Methods: Balb/c mice were treated with single dose of streptozocin $(240 \mathrm{mg} / \mathrm{kg})$ to induce diabetes and compared with control group, which was treated with citric buffer $(\mathrm{pH}=4.5)$. After 3 days, the blood glucose level was measured to confirm the induction of diabetes. Normalization of blood glucose level in diabetic mice was achieved after $0.1 \mathrm{ml} / \mathrm{kg}$ Mixtard@ insulin therapy for more 5 days. Then, the mice livers were isolated to extract RNA and convert it to cDNA. The gene expression of 14 genes, which play a major role in drug and arachidonic acid metabolism, were measured using quantitative real-time polymerase chain reaction technique.

Results: It is found that the gene expression was down-regulated (t-test, p value $<0.05$ ) in the livers of UDM mice. The most down-regulated genes were cyp4a12, cyp1a2 and slc22a1 with more than 10 fold reduction. The livers of CDM mice showed significantly (t-test, $\mathrm{p}$ value $<0.05$ ) higher levels of mRNA than UDM mice, but still lower than the controlled non-diabetic mice.

Conclusion: This study concluded that hepatic gene expression of drug metabolizing and arachidonic acid- cyp450 enzymes is reduced in insulin-dependent diabetic mice, which can explain, at least in part, the variation in drug and fatty acid metabolism between healthy people and diabetic patients.

References: Irshaid, Y., H. al-Hadidi, M. Abuirjeie, A. Latif, O. Sartawi, and N. Rawashdeh. 1992. Acetylator phenotypes of Jordanian diabetics. Eur J Clin Pharmacol 43: 621-623., Dostalek, M., M. H. Court, B. Yan, and F. Akhlaghi. 2011. Significantly reduced cytochrome P450 3 A4 expression and activity in liver from humans with diabetes mellitus. Br J Pharmacol 163: 937-947., Dostalek, M., M. H. Court, S. Hazarika, and F. Akhlaghi. 2011. Diabetes mellitus reduces activity of human UDP-glucuronosyltransferase 2B7 in liver and kidney leading to decreased formation of mycophenolic acid acyl-glucuronide metabolite. Drug Metab Disp, Lee, J. H., S. H. Yang, J. M. Oh, and M. G. Lee. 2010. Pharmacokinetics of drugs in rats with diabetes mellitus induced by alloxan or streptozocin: comparison with those in patients with type I diabetes mellitus. J Pharm Pharmacol 62: 1-23.

Keywords: Drug metabolism, gene expression, diabetes, insulin, arachidonic acid

\section{Sphingosine-kinase 1 and epithelio-mesenchymal transition in lung adenocarcinomas}

\section{Pierre Fouret}

Pathology, APHP, Paris, France

By comparing expression profiles with copy-number aberrations in lung adenocarcinomas, we have identified sphingosine-kinse 1 (SPK1) as a likely driver of an epithelio-mesenchymal transition (EMT). SPK1 expression was associated both with SPK1 copy-number gains and with an EMT signature in lung adenocarcinomas with non-lepidic histological subtypes. Expression of SPK1 and EMT-associated transcription factors were localized to tumor cells. In A549 and NCI-H1792 cells, SPK1 overexpression or exposure to its product sphingosine-1-phosphate (S1P) led to a decreased expression of E-cadherin (CDH1) mRNA, which indicated passage through an EMT. However, other lung cancer cells with a pure epithelial profile were unresponsive to SPK1 or S1P. Our data show that SPK1 can be an important factor of an EMT, which has been associated with metatasis and resistance to drugs in many cancers. However, response to SPK1 or S1P is modulated by other tumor cell characteristics, including possible epigenetic modifications. Identifying such modifications would allow the development of assays that would be helpful to determine whether lung adenocarcinoma treatment can be improved by drugs targeting SPK1 or the receptors that are involved in S1P signaling.

\section{Aqueous Extract Of Selected Wild Vegetables Reduce Blood Pressure And Improve Cardiac Functions In Isoproterenol- Induced Myocardial Infarction Male Rats}

\section{Kehinde Bolarinwa KA}

Department of Cell Biology and Genetics, University of Lagos, Lagos, Nigeria

Background: A rising trend in heart disease such as myocardial infarction (MI) - an important and common cardiovascular disease has been attributed to lower or non-consumption of vegetables in most developing countries (Inegbenebor, 2013). 
Objective: This study was designed to evaluate the effect of selected 5 wild vegetables (Basella. alba (BA), Crassocephalum crepidioides (CC), Launaea taraxacifolia (LT), Senecio biafrae (SB) and Solanum nigrum (SN)) on blood pressure (BP) and cardiac functions in male rats treated with isoproterenol (ISO) for induction of M.

Design: Dried ground leaves (350g each) of BA, CC, LT, SB and SN were each extracted at room temperature (260C) with 3 litres of water using maceration techniques. Rats $(150 \mathrm{~g}-200 \mathrm{~g})$ were randomly divided into 14 groups of 6 rats each. They were fed with rat chows, water and vegetable extract for 28 days. Groups 1 and 8 served as normal and ISO controls respectively while groups 2 to 6 received 200 mg kg-1b.wt. of each vegetable. Groups 9-14 were pretreated with each vegetable extract (200 mg kg-1b.wt.) and MI was induced with ISO (20 mg kg-1b.wt; subcutaneously) on days 29 and 30. Group 7 received a combination of all the extracts in ratio 1:1:1:1:1. Group 14 received extract combination and ISO. Thereafter, BP was measured via arterial cannulation. Lactate dehydrogenase (LDH), C-reactive protein (CRP), Creatine kinase (CK) and Aspartate aminotransferase (AST) were measured in cardiac blood samples.

Results: CRP, LDH, CK, AST and BP fell $(\mathrm{p}<0.05)$ in all vegetable pre-treated groups with mixture group displaying the lowest activities of CRP. Pretreatment with vegetables abolished the increases in CRP, LDH, CK, AST and BP $(p<0.05)$ due to ISO.

Conclusions: Results suggest that the selected vegetable extracts reduced BP and improve cardiac functions in ISO-induced MI male rats. References: Inegbenebor U. Sudden Cardiac Death In Nigeria: Pathophysiology And Evidence Based Intervention Strategy. IJBAIR 2013; 2(4): $73-77$, ,

Keywords: Basella alba, Crassocephalum crepidioides, Launaea taraxacifolia, Senecio biafrae, Solanum nigrum, Myocardial infarction

\title{
Relationship between metabolic syndrome and dry eye symptoms
}

\author{
Malgorzata Mrugacz, Anna Bryl \\ Department of Ophthalmology and Eye Rehabilitation, Medical University, Bialystok, Poland
}

Background: With a 35\% prevalence in the general population, dry eye disease (DED) is a prevalent public health issue that significantly affects quality of life. Dry eye is a multifactorial disease of the tears and ocular surface that results in the following symptoms: discomfort, visual disturbance, and tear film instability with potential damage to the ocular surface. Many diseases have been associated with DED, including metabolic syndrome (MetS).

Objective: To evaluate dry eye disease in patients with metabolic syndrome and compare with healthy individuals. Design: 60 eyes of 30 patients with metabolic syndrome (group 1) and 54 eyes of 30 healthy individuals (group 2) were included in this cross-sectional study. All participants were evaluated using the Ocular Surface Disease Index (OSDI) questionnaire and dry eye tests: Schirmer I test and tear film breakup time (TBUT).

Results: The Schirmer test values and TBUT in group $1(10 \pm 2.7 \mathrm{~mm}$ and $13.8 \pm 3.6 \mathrm{sec}$, respectively) were significantly lower compared with group $2(16.4 \pm 2.7 \mathrm{~mm}$ and $18.5 \pm 0.5 \mathrm{sec}$, respectively).

Conclusions: Our findings highlight that metabolic syndrome can influence tear film function. Patients with metabolic syndrome have tear film disturbances. Clinicians should be aware of higher DED incidence in patients with MetS for early treatment to prevent serious ocular complications.

Ma A, Mak MS, Shih KC, Tsui CK, Cheung RK, Lee SH, et. Al. Clin Exp Ophthalmol 2018;18: doi: 10.1111/ceo.13146.

References: Ma A, Mak MS, Shih KC, Tsui CK, Cheung RK, Lee SH, et. Al. Clin Exp Ophthalmol 2018;18: doi: 10.1111/ceo.13146.

Keywords: metabolic syndrome, dry eye, ocular surface

\section{Monitoring of myelodysplastic syndrome progression based on lectin array and analysis of protein interactions}

Leona Chrastinova 1, Ondrej Pastva 1, Marketa Bockova 2, Jaromir Novak 1, Jana Stikarova 1, Alzbeta Hlavackova 1, Jiri Suttnar 1, Roman Kotlin 1, Jiri Homola 2, Jaroslav Cermak 1, Jan Dyr 1

1. Department of Biochemistry, Institute of Hematology and Blood Transfusion, Prague, Czech Republic

2. Department of Optical Biosensors, Institute of Photonics and Electronics, Prague, Czech Republic

Background: Myelodysplastic syndromes (MDS) are a heterogeneous group of hematological malignancies with high risk of progression into acute myeloid leukemia. In a majority of cancers the fucosylation and sialylation expression are found to be significantly modified. Therefore, using selected lectins these aberrations in glycan structures can be utilized as targets to improve existing cancer biomarkers. Surface plasmon resonance (SPR) method is an optical label-free technique that allows observing and quantifying the target biomolecules in real time [1].

Objective: The aim of this study was to develop lectin chip for discrimination among different MDS patient subgroups and healthy controls using SPR biosensor.

Design: The protein interactions were studied using protein chips comprising selected lectins and an SPR biosensor. Six lectins with specific binding affinities for different sugars were chosen. The blood plasma of healthy controls and MDS patients was analyzed. Patients were divided into four subgroups: refractory anemia with ringed sideroblasts (RARS), refractory cytopenia with multilineage dysplasia (RCMD), refractory anemia with excess blasts (RAEB), and patients who progressed to acute myeloid leukemia (AML). The interacting plasma proteins were eluted from chip and identified by mass spectrometry. 
Results: The differences of SPR responses were statistically significant for half of tested lectins. Using these lectins, the RAEB subgroup was likely to be distinguished from RARS, RCMD, and AML patients.

Conclusions: This study demonstrates that even a small lectin array allows the SPR method to discriminate among different MDS subgroups. Acknowledgements: This work was supported by the Ministry of Health, Czech Republic, no. 00023736, by the Academy of Sciences, Czech Republic no. P205/12/G118, by ERDF OPPK CZ.2.16/3.1.00/24001 and by the European Regional Development Fund and the state budget of the Czech Republic (project AIIHHP: CZ.02.1.01/0.0/0.0/16_025/0007428, OP RDE, Ministry of Education, Youth and Sports).

References: Homola J. Chem Rev. 2008 Feb;108(2):462-93

\title{
Association of beta-adrenergic receptors polymorphisms with Body Mass Index in a Southeastern European Caucasian population
}

\author{
Aikaterini Karathanasopoulou, Giwrgos Vlachos, Athanasios Mouchtaris-Michailidis, Maria Papasavva, Angeliki Andrianopoulou, Martha- \\ Spyridoula Katsarou, Maria Lagiou, Nikolaos Drakoulis \\ Faculty of Pharmacy, School of Health Sciences, National and Kapodistrian University of Athens, Athens, GREECE
}

Background: Beta-adrenergic receptors (ADRB) play a major role in the regulation of body fat, as their activation promotes lipolysis and thermogenesis. Genetic polymorphisms of these receptors, rs1801252, rs1801253 (ADRB1), rs1042713, rs1042714 (ADRB2) and rs4994 (ADRB3), have been associated with Body Mass Index (BMI) and obesity, with contradictory results reported among different ethnic groups. However, in the majority of literature studies, a significant association between these polymorphic variants and weight gain has been shown.

Objective: Aim of the present study is to investigate the correlation of the aforementioned beta-adrenergic receptor polymorphisms with BMI and obesity in a Southeastern European Caucasian population.

Design: A sample of 332 subjects volunteered to participate in the present genotyping analysis, after signing a written inform of consent. Each subject was classified to one of the following groups: 1) normal weight, 2) overweight and 3) obese, based on its corresponding BMI value. DNA was extracted from buccal swabs and genotyping was performed using real-time PCR (Light Cycler 480, Roche, Germany). Data were analyzed with IBM Statistics SPSS 22.0 package.

Results: Statistical analysis showed significant association of body weight gain with rs1801252 polymorphism between normal and overweight subjects, as well as with rs1801253 among obese subgroups (p-value $<0.05)$. Rs1042713 and rs1042714 (p-value <0.05) were correlated with elevated BMI among overweight and obese subgroups. The rest comparisons showed no statistically significant results.

Conclusions: Beta-adrenergic receptor polymorphisms seem to affect body weight gain in Southeastern European individuals. Thus, genotyping for these polymorphisms might provide a better understanding on the development of obesity and can help in the fight for disease prevention. Further studies should be conducted to determine their impact on obesity in other ethnic groups, as well.

Acknowledgements: This research was supported by the research group of Clinical Pharmacology and Pharmacogenetics at the National and Kapodistrian University of Athens. All the authors report no conflict of interest.

References: Masuo K, Lambert GW. Relationships of adrenoceptor polymorphisms with obesity. J Obes. 2011;2011

\section{Tryptophan metabolites and oxidative stress in patients with cardiovascular disease or stroke}

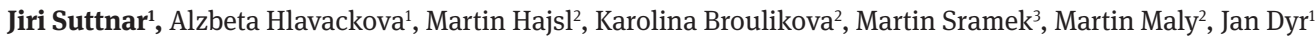

1. Department of Biochemistry, Institute of Hematology and Blood Transfusion, Prague, Czech Republic

2. Department of Medicine, 1st Faculty of Medicine, Central Military Hospital, Prague, Czech Republic

3. Comprehensive stroke Center, Central Military Hospital, Prague, Czech Republic

Background: Several hypotheses have suggested that various factors, including oxidative stress, immune activation, and inflammation, are central to the pathogenesis of atherosclerosis, cardiovascular disese and stroke. Tryptophan and some metabolites may act as antioxidants that can remove reactive oxygen species effectively (1).

Objective: The aim of this study was to study relationship of tryptophan metabolites and oxidative stress in plasma samples of patients with cardiovascular disease or stroke.

Design: The EDTA plasma was from 5 groups of patients (each group consisted of 25 probands): patients with acute coronary syndrome (ACS), with normal coronary angiogram (NCA), with atherosclerotic carotides (C), with stroke (S), and control healthy donors (K). Tryptophan metabolites (12), neopterine, and riboflavin were analyzed after plasma proteins precipitatation with methanol by liquid chromatography coupled to tandem mass spectrometer (LC-MS/MS). As a criterion of oxidative stress, the total malondialdehyde (MDA) concentration in plasma was analyzed using derivatization with 2,4-dinitrophenylhydrazine and subsequent LC-MS/MS.

Results: We found significant changes in concentration of malondialdehyde and 11 tryptophan metabolites (not for quinolinic acid) in all groups of patients. Neopterin as a marker of immune activation was significantly increased in ACS and S patients as compared with controls and negatively correlated with tryptophan concentration. We found no relationship between riboflavin and tryptophan metabolism.

Conclusions: Oxidative stress in both groups of patients, cardiovascular diseses and stroke was accompanied with dysregulated tryptophan metabolic pathways. Metabolomic approach thus seems to be usefull for better understanding of the pathogenesis of cardiovascular and cerebrovascular diseases. 
Acknowledgements: This work was supported by the European Regional Development Fund and the state budget of the Czech Republic (project AIIHHP: CZ.02.1.01/0.0/0.0/16_025/0007428, OP RDE, Ministry of Education, Youth and Sports), by the project of the Ministry of Health, Czech Republic 00023736, by Grant from the Ministry of Health NV18-08-00149, by Grant from the Academy of Sciences, Czech Republic, P205/12/G118 and by ERDF OPPK CZ.2.16/3.1.00/24001.

References: Wang Q, Liu D, Song P, Zou M-H. Front Biosci 2015; 20: 1116-1143

\section{Phthalates exposure influence on obesity development and glucose homeostasis}

Natasa Milosevic ${ }^{1}$, Maja Milanovic ${ }^{1}$, Kristina Stepanovic ${ }^{1}$, Stefan Stojanoski ${ }^{1}$, Dragana Bosic Zivanovic ${ }^{2}$, Natasa Milic ${ }^{1}$, Milica Medic Stojanoska ${ }^{1}$

1. University of Novi Sad, Faculty of Medicine, Novi Sad, Serbia

2. Health Center Ruma, Ruma, Serbia

Introduction: Phthalates, identified as endocrine disruptors, are widely used as plasticizer in many everyday products and therefore may cause adverse health effects, such as disruption of lipid and glucose homeostasis.

Objective: The aim of the study was to evaluate the frequency of the detection as well as urinary levels of monoethyl phthalate (MEP), mono(2-ethylhexyl) phthalate (MEHP) and sum of phthalate metabolites in obese patients, patients with diagnosed type 2 diabetes mellitus (T2DM) and normal weight healthy volunteers.

Methods: The study was conducted on 305 volunteers (158 male and 147 female) divided into three groups: I-obese with central obesity, II-patients with diagnosed T2DM treated only with alimentation therapy and III-control, normal weight healthy volunteers. The concentration of phthalate metabolites was determined in the morning spot urine using gas chromatography coupled with mass spectrometry.

Results: At least one of the phthalate metabolite was detected in 49.84\% (152/305) subjects enrolled in this study with no statistical differences among subgroups: 52.88\% (55/104) in obese, 52.48\% (53/101) in T2DM patients and 44\% (44/100) in normal weight subgroup, nor among genders 50.63\% (80/158) in male and 48.98\% (72/147) in female subjects. Although no statistically significant, higher phthalate sum levels were observed among obese (333.74 \pm 815.48$)$ and T2DM (404.16 \pm 1017.15$)$ subgroups in comparison with the control subgroup (148.04 \pm 188.72$)$ with the highest detected concentration in a T2DM patient. The urine samples from 66 volunteers (out of 305) were positive on MEP. Statistically significant higher levels of MEP were obtained in obese $(\mathrm{p}=0.005)$ and normal weight (control) group $(\mathrm{p}=0.05)$ in comparison with patients with T2DM. Also, MEHP was determined in 72 out of 305 urine samples with higher frequency of the detection among obese and T2DM volunteers in comparison with control subgroup ( $\mathrm{p}=0.047$ and $\mathrm{p}=0.032$, respectively).

Conclusions: Based on the obtained results, MEP and MEHP may influence obesity development and MEHP may affect glucose homeostasis.

Acknowledgements: Grant No. 114-451-2216/2016, Provincial Secretariat for Science and Technological Development, APV, Serbia.

References: Miloševic N, Milic N, Živanovic Bosic D, Bajkin I, Percic I, Abenavoli L, Medic Stojanoska M. Environ Monit Assess 2018; 190:1-17 Keywords: phthalates, obesity, glucose homeostatis

\section{Inflammation Related TNF- $\alpha$, IL-6 and CRP Gene Polymorphisms in a Southeastern European Caucasian Population Sample and Comparison with Other Populations}

Maria Papasavva, Evgenia Georgiadi, Alexandra Petraina, Martha-Spyridoula Katsarou, Maria Lagiou, Panagiota Stamou, Thodoris Kalogridis, Nikolaos Drakoulis

Research Group of Clinical Pharmacology and Pharmacogenomics, Faculty of Pharmacy, School of Health Science, National and Kapodistrian University of Athens, Athens, GREECE

Background: Inflammation is a complicated, well-orchestrated process by a variety of cells and chemical mediators such as TNF-a, IL-6 and CRP. The less common A allele of TNF-a gene polymorphism rs1800629 seems to increase TNF-a expression levels. The polymorphic allele C of IL-6 gene polymorphism rs1800795 and T allele of CRP gene polymorphism rs1205 are related to lower levels of IL-6 and CRP, respectively.

Objective: A frequency distribution analysis of rs1800629, rs1800795 and rs1205 polymorphisms in a South Eastern European Caucasian (SEC) population sample was performed and the results were compared with those of other populations through ensembl database.

Design: DNA was extracted from buccal swabs of 852 SECs and analysed with real time PCR (Light Cycler 480, Roche, Germany) using appropriate LightSnip Assays (TIBMOLBIOL, Germany) and melting curve analysis. Statistical analysis was performed with IBM Statistics SPSS 22.0 package.

Results: Gene distribution of rs1800629 was $\mathrm{G}: \mathrm{G}=81.8 \%, \mathrm{G}: \mathrm{A}=17.4 \%$ and A:A=0.8. The wild-type $\mathrm{G}$ allele frequency was $90.5 \%$. Frequencies of rs 1800795 were $\mathrm{G}: \mathrm{G}=54.8 \%, \mathrm{G}: \mathrm{C}=38.7 \%$ and $\mathrm{C}: \mathrm{C}=6,5 \%$. The wild-type $\mathrm{G}$ allele frequency was $74.2 \%$. The frequencies of rs $1205 \mathrm{were}$ $\mathrm{C}: \mathrm{C}=47.7 \%, \mathrm{C}: \mathrm{T}=41.9 \%$ and $\mathrm{T}: \mathrm{T}=10.4 \%$. The wild-type $\mathrm{C}$ allele frequency was $68.6 \%$. The frequency distribution of all analysed SNPs of the SEC population differs significantly when compared with other populations (SEC v/s Europeans (P-value $<0.001)$, Africans $(\mathrm{P}$-value $=0.03)$, East Asians (P-value $<0.001)$ and South Asians (P-value $<0.001)$ for rs1800629, v/s global (P-value $<0.001)$, Europeans $(\mathrm{P}$-value $<0.001)$, 
Africans (P-value <0.001), East Asians (P-value <0.001) and South Asians (P-value $<0.001)$ for rs1800795 and v/s Africans (P-value <0.001) and East Asians (P-value<0.001) for rs1205).

Conclusions: Inflammation is affected by multiple factors; however, the genotyping analysis of polymorphisms may play a significant role in susceptibility of inflammation. SEC population differs from other populations maybe because of the geographical position of SEC, on the crossroad between three continents.

Acknowledgements: This research was supported by the research group of Clinical Pharmacology and Pharmacogenetics at the National and Kapodistrian University of Athens. All the authors report no conflict of interest.

\section{Population Based Analysis of GSTP1 Gene Polymorphisms Distribution In A Southeastern European Caucasian Population Sample And Comparison With General European Population Frequency Distributions}

Konstantina Doulou, Evangelia Alexiou, Martha Katsarou, Rozana Latsi, Maria Papasavva, Maria Lagiou, Nikolaos Drakoulis Research Group of Clinical Pharmacology and Pharmacogenomics, Faculty of Pharmacy, School of Health Sciences, National and Kapodistrian University of Athens, Athens, GREECE

Background: Glutathione S-transferase P1 (GSTP1) is a member of the GSTs superfamily that protects the body from carcinogenic or genotoxic compounds. GSTP1 polymorphisms may contribute to the development of several diseases, including cancer. The enzyme's activity can be affected by two SNPs (rs1695 and rs1138272) that lead to amino acid substitutions which alter its ability to respond to reactive oxygen and/or nitrogen species' (ROS/RNS) activity, the causal factor of many diseases.

Objective: A frequency distribution analysis of GSTP1 rs1695 and rs1138272 in a Southeastern European Caucasian (SEC) population sample was made and a comparison with other European (EUR) populations including Italian-Toscani (TSI), Finnish (FIN), British (GBR), Iberian (IBS) and residents of Utah with Northern and Western European ancestry (CEU) followed. (data obtained from ensembl.com)

Design: DNA from buccal swabs of 943 healthy, non-related SEC volunteers was collected and analyzed by real-time LightCycler 480 PCRplatform (Roche, Germany) using appropriate LightSnip Assays (TIBMOLBIOL, Germany).

Results: The frequency of the wildtype A allele of rs 1695 was $72.9 \%$. Out of the 943 SECs, $54.0 \%$ were homozygous for the wildtype genotype (A:A), 38.0\% were heterozygous (A:G) and 8.0\% were homozygous for the $G$ allele (G:G). Additionally, $G$ allele appeared 1.3 times more in Europeans than in SECs (OR =1.3314, $\mathrm{p}=0.0007)$. Regarding rs1138272, 88.8\% were homozygous for the wildtype genotype (C:C), $10.7 \%$ were heterozygous (C:T) and only $0.5 \%$ were homozygous for the T allele (T:T). The frequency of the wildtype C allele was $94.1 \%$. No statistically significant difference in frequency distribution of this polymorphism was observed between SECs and Europeans. Both polymorphisms were in Hardy-Weinberg equilibrium (x2=1.64 for rs1695 and x2=1.04 for rs1138272).

Conclusion: As a phase II drug metabolizing enzyme, GSTP1 serves as a catalytic agent for GSH's conjugation to a variety of electrophilic substrates, an inactivating agent of a variety of antineoplastic and other drugs and an activator of antineoplastic prodrugs. Thus, GSTP1 polymorphisms may be further investigated as pharmacotherapy biomarkers of various diseases.

\section{Cardiovascular disease biomarker discovery using network biology}

Karla V. Allebrandt', Fancesca Frau ${ }^{1}$, Sibylle Hess² ${ }^{2}$ Hertzel Gerstein ${ }^{3}$, Daniel Crowther ${ }^{1}$, Guillaume Pare

1. Translational Informatics, R\&D Trans. Med. \& Early Development, Sanofi-Aventis, Frankfurt, Germany

2. Biomarkers and Clinical Bioanalysis, Sanofi-Aventis, Frankfurt, Germany

3. McMaster University and Population Health Research Institute, Hamilton, Canada

Background: due to the complexity of cardiovascular disease (CVD), the ability of single markers to predict CV outcome (CVO) risk is limited, though many genetic loci and protein biomarkers (BMKs) have been discovered. The integration of such results with prior knowledge on biological pathways, gene function and interaction from large databases can enhance the understanding of CVD progression, revealing underlying molecular networks.

Objective: we used network biology to identify links between CVO risk markers and to discover novel central BMK involved in the disease mechanism.

Design: we built a CVD network making use of topological characteristics of human protein interaction networks to identify one-step away direct regulators of the dataset that are statistically overconnected with the objects from the data set. These were 16 protein BMKs and, 8 genes corresponding to loci associated with CVO, both previously identified in the ORIGIN CVO trial BMK (1) and genome-wide association (GWA) sub-studies. To validate the findings, we re-ran these analyses replacing genetic associated genes by other 90 genes from an independent large CVO GWA meta-analysis from the CARDIOGRAM consortium.

Results \& discussion: as a proof of concept, the network biology results pointed to 14 (12 in the replication study) out of the 16 BMKs that were identified by standard BMK analyses as being part of a main network, though many other candidates were fed as input to the network calculations. The novelty of the results relies on the connectivity network and, the central nodes that were discovered. These were TNF-alpha, IL6, and RelA NF-KB subunit, which thereby mechanistically connect most CVO risk BMKs. RORA \& GHR, which had borderline significant alleles associated with CVOT in the ORIGIN GWA, were also interaction partners of the central nodes identified. 
Conclusions: compared with traditional BMK analysis, this approach can provide mechanistic insights (also to GWAs identified genes) to disease development and point to main upstream regulators for the relevant pathway. Furthermore, the network biology can be further explored by using single level patient data to understand its impact on disease progression or response to therapeutic intervention.

References: Gerstein et al. 2015, Circulation. 132(24):2297-304.

Keywords: Network biology, Biomarker discovery, Systems Medicine, CVO risk factors, \& GWAs.

\section{Systems biology analysis of interaction and regulation networks in high throughput RNAi screenings}

Ghislain Bidaut ${ }^{1}$, Quentin Da Costa ${ }^{1}$, Claire Rioualen ${ }^{2}$, Bernard Chetrit ${ }^{1}$, Emmanuelle Charafe-Jauffret ${ }^{1}$, Christophe Ginestier $^{1}$

1. Centre de Recherche en Cancérologie de Marseille, Inserm U10681; CNRS UMR72582; Aix-Marseille Université; Institut Paoli-Calmettes, 13009 Marseille, France

2. Technological Advances for Genomics and Clinics (TAGC) Inserm U1090; Aix-Marseille Université, 13009 Marseille, France

Background: High-throughput RNAi screenings (HTS) allow quantifying the impact of the deletion of each gene for any biological function, from virus-host interactions to cell differentiation. However, there has been little development for dedicated functional or systems biology analysis tools to characterize genes modules identified in the screen.

Objective: Our goal is the identification of gene regulatory modules impacted in high-throughput screenings by integrating transcription factors-target genes interaction data ((TF-DNA interactions) and protein-protein interaction networks (PPI interactome) on top of screening z-scores.

Methods: The High-Throughout Screening Network algorithm (HTS-Net) (1) identifies functional genes modules and their regulators using a search heuristics that works in parallel on a protein-protein interaction network and a regulation network. We superimpose RNAi-based gene scores on the interaction and the regulation maps separately. Each one of them is then searched for high scoring areas. The identified regions of interest, the subnetworks, are extracted and merged to form meta-subnetworks that incorporate regulation information and PPIs. Statistical validation ensures high performance. This approach allows reprioritizing genes by replacing hits into their biological context, which includes their physical interactors and their regulators. HTS-Net produces complete HTML reports for subnetwork exploration and analysis.

Results: The HTS-Net algorithm was evaluated on three RNAi screen studies (Stem cell differentiation, cancer stem cell regulators and host HCV interactions) for which we reported newly found markers and pathways, and comparison of identified genes with the original analyses. We propose HTS-Net to the community as a web service at http://htsnet.marseille.inserm.fr/. We also compared HTS-Net with two other network analysis algorithms, including CARD, software dedicated to RNAi screening functional analysis and Hotnet2, a general-purpose gene network analysis program.

Conclusions: HTS-Net proves better performance than simple gene rankings by z-scores, by re-prioritizing genes and replacing them in their biological context, as shown by the three studies that we reanalyzed.

Acknowledgements. Support to this project is coming from the Institut National du Cancer grant Number INCA_5911 to CG.

References: Rioualen C, Da Costa Q, Chetrit B, Charafe-Jauffret E, Ginestier C, Bidaut G. HTS-Net: An integrated regulome-interactome approach for establishing network regulation models in high-throughput screenings. PloS One. 2017;12(9):e0185400.

Keywords: Systems biology, RNAi screening, gene regulation, gene networks, bioinformatics

\section{SiMS score in obese children, adolescents and youth increases with age}

Vesna Dimitrijevic-Sreckovic ${ }^{1}$, Hristina Janeski ${ }^{2}$, Branko Sreckovic ${ }^{3}$, Mirjana Popadic ${ }^{4}$, Ivan Soldatovic ${ }^{5}$

1. Diabetology, Clinic for Endocrinology, Diabetes and Metabolic Diseases, Clinical Center of Serbia; Faculty of Medicine, Belgrade University, Serbia, Belgrade, Serbia

2. Pediatrics, University Children's Hospital, Belgrade, Serbia

3. Internal medicine, General Hospital Aurora, Belgrade, Serbia

4. Clinic for Endocrinology, Diabetes and Metabolic Diseases, Clinical Center of Serbia, Belgrade, Serbia

5. Statistics, Institute for Medical Statistics and Informatics, Faculty of Medicine, Belgrade University, Serbia, Belgrade, Serbia

Background: In obese patients, abdominal obesity is accompanied with hyperinsulinism, insulin resistance (IR), decreased insulin sensitivity, hyperhomocysteinemia, microalbuminuria, increased thrombotic and inflammatory factors and decreased antioxidant status. Aforenamed are also markers of endothelial dysfunction seen in patients with metabolic syndrome (MS). IR results in fat deposition in the liver and occurrence of non-alcoholic fat liver disease (NAFLD).

Objectives: This study analyzes siMS score, as a method for quantification of MS, prediabetes, IR, thrombotic and inflammatory factors, NAFLD, microalbuminuria, homocysteine and antioxidant status in obese children, adolescents and youth.

Methods: The study included 205 obese individuals with pre-MS and MS (age 7-30) classified according to age I-children (7-15), II-adolescents (16-20) and III-youth (20-30). IDF classification was applied for diagnosing MS. Patients with less than three criteria indicated below were considered pre-MS. siMS risk score by Soldatovic I et al 2016 was used. IR was determined by homeostatic model assessment (HOMA-IR). Activities of markers of antioxidant defense, superoxide dismutase (SOD) and glutathione peroxidase (GPX) were determined in erythrocytes. 
Results: siMS score increases considerably with age, (I- 2.71 \pm 0.49 , II- $2.81 \pm 0.90$, III- 3.18 $\pm 0,89$ ). Patients had increased waist circumference (WC), blood pressure, insulin mean value, HOMA-IR, triglycerides, CRP, PAI-1, microalbuminuria and decreased HDL, SOD and GPX. Prediabetes increases with age, IFG (I- 2.1, II- 10.8, III- 13.8\%) and IGT (I- 4.2, II- 4.0, III- 5.5\%). Correlations: HOMA-IR with BMI, WC, blood pressure, triglycerides, basal, $30 \mathrm{~min}, 120 \mathrm{~min}$ and mean insulinemia, basal glycemia $(\mathrm{p}<0.01)$, PAI- $(\mathrm{p}<0.05), \mathrm{CRP}(\mathrm{p}<0.01)$ and negatively with HDL $(\mathrm{p}<0.01)$. Conclusion: Correlations of HOMA-IR with WC, PAI-1 and CRP explain connection between IR and abdominal obesity, increased levels of thrombotic and inflammatory factors and early risks of atherosclerosis. Abdominal obesity, hyperinsulinism, hypertension, hypertrygliceridemia, prediabetes, IFG, IGT, NAFLD, so as co-founding factors of MS, CRP, PAI-1, microalbuminuria, homocysteine increases with age in investigated groups of children, adolescents and youth. siMS score, as a method for quantification of MS, increases with age, displaying increasing of co-founding factors of MS.

References: Soldatovic I, Vukovic R, Culafic D, Gajic M, Dimitrijevic-Sreckovic V. siMS Score: Simple Method for Quantifying Metabolic Syndrome. PLoS One. 2016 Jan 8;11(1):e0146143

Keywords: siMS score, metabolic syndrome, obesity, prediabetes, insulin resistance, homocysteine

\section{Confounding factors of metabolic syndrome- confirmed by siMS score and siMS risk score}

Branko Sreckovic ${ }^{1}$, Vesna Dimitrijevic- Sreckovic ${ }^{2}$, Hristina Janeski ${ }^{3}$, Emina Colak ${ }^{4}$, Nenad Janeski ${ }^{5}$, Ivan Soldatovic ${ }^{6}$

1. Internal Medicine, General Hospital Aurora, Belgrade, Serbia

2. Diabetology, Clinic for Endocrinology, Diabetes and Metabolic Diseases, Clinical Center of Serbia, Faculty of Medicine, Belgrade University, Serbia, Belgrade, Serbia

3. Pediatrics, University Children'sHospital, Belgrade, Serbia

4. Biochemistry, Institute of Medical Bichemistry, Belgrade, Serbia

5. Radiology, Clinical Center Zemun, Belgrade, Serbia

6. Statistics, Institute for Medical Statistics and Informatics, Faculty of Medicine, Belgrade University, Serbia, Belgrade, Serbia

Background: Patients with metabolic syndrome (MS) have increased abdominal obesity, hypertension, hypertrigliceridemia, inflammation factors, insulin resistance (IR), homocysteine and microalbuminuria as markers of endothelial dysfunction. A correlation between homocysteine and hypertension and hyperlipoproteinemia showed that homocysteine could be used as a potential marker for atherosclerosis progression. IR results in fat deposition in the liver and occurrence of non-alcoholic fat liver disease (NAFLD).

Objectives: To examine corelation of siMS score and siMS risk score with IR as with other co-founding factors of MS, NAFLD, inflammation and thrombotic factors, uric acid, and homocysteine.

Methods: The study included overweight and obese patients ( $\mathrm{n}=148$ ), age 30-75 years, classified in two groups: I- with MS (68 patients); IIwithout MS (80 patients). IDF classification was applied for diagnosing MS. siMS score, as a method for quantification of MS, siMS risk score for assesing atherosclerotic complications, by Soldatovic I et all 2016 were used. IR was determined by homeostatic model assessment (HOMA IR). Homocystein was determined by immunoassay FPIA-Abbot.

Results: Patients with MS had increased waist circumference (WC), BMI, blood pressure, $(p<0.001)$ glycaemia $(p=0.006)$, and insulin in 0 min $(p=0.007)$ and insulin in $120 \mathrm{~min}(\mathrm{p}=0.014)$ OGTT, HOMA IR $(\mathrm{p}=0.002)$, triglycerides, CRP, Apo B $(\mathrm{p}=0.01), \mathrm{uric}$ acid $(\mathrm{p}<0.001)$, ALT $(p=0.007)$ and GGT $(P<0.001)$ and decreased HDL $(p<0.001)$. siMS score correlates significantly with CRP, uric acid, HOMA IR, GGT $(p<0.001)$, ALT $(p=0.001)$, platelates $(p=001)$, fibrinogen $(p=0.005)$. siMS risk score shows a positive correlation with homocysteine $(0.015)$. Conclusion: Correlation of siMS score with HOMA-IR confirmed that insulin resistance is in the basis of MS. Correlation of siMS-score with parameters of NAFLD (ALT and GGT), CRP, uric acid, thrombotic factors (platelates, fibrinogen) indicated that they are significant confounding factors of MS. SiMS risk score correlation with homocysteine indicated that hyperhomocysteinemia increases with age.

References: B Sreckovic, I Soldatovic, E Colak, I Mrdovic, M Sumarac-Dumanovic, H Janeski, N Janeski, J Gacic i V Dimitrijevic Sreckovic. Homocysteine is the confounding factor of metabolic syndrome-confirmed by siMS score, Drug Metaboll Pers Ther 2018

Keywords: metabolic syndrome, obesity, siMS score, siMS risk score, homocysteine

\section{Framingham coronary heart disease risk does not correlate with coronary calcium burden in patients with essential thrombocytosis}

\section{Matjaž VRTOVEC}

Angiology clinic, University Clinical Center Ljubljana, Ljubljana, SLOVENIA

Atherosclerosis is chronic inflammatory disease of arterial wall. Cardiovascular diseases, associated with atherosclerosis, represent a major healthcare problem in the developed and developing world. Philadelphia-negative myeloproliferative diseases (MPN's) are group of chronic hematogenous disorders with acquired transformation of hematopoietic stem-cells as a central mechanism. Chronic inflammation has an important role in this mechanism. MPN's are connected with higher cardiovascular morbidity and mortality. Our hypothesis was, that chronic inflammation as a trigering mechanism of both processes could cause earlier decline of endothelial function and cause greater arterial stiffness, promote earlier asymptomatic atherosclerotic changes at the patients with Philadelphia-negative mieloproliferative disorders. Our objective was to determine if patients with essential thrombocytosis (ET), a subtype of MPNs, free of symptomatic atherosclerosis, 
have greater carotid artery stiffness, worse endothelial function, greater coronary calcium and carotid plaque burden than control subjects. 40 ET patients without overt vascular disease, and 42 apparently healthy, age and sex matched control subjects with comparable classical risk factors for atherosclerosis and Framingham risk of coronary disease were enrolled. All subjects were examined by physical and laboratory testing, carotid Echo-tracking ultrasound, digital EndoPat pletysmography and CT coronary calcium scoring.

No differences were found between the two groups in the carotid plaque score, carotid artery stiffness or the estimated pulse wave velocity. The RHI of the digital arteries showed a trend towards toward better endothelial reactivity in control subjects [2.35 (0.62) vs. 2.10 (0.57)], but the difference was not significant $(\mathrm{p}=0.07)$. There were also no differences in the AI, an estimate of stiffness of the conductive arteries of the upper limb. The majority of study subjects had no calcification on coronaries, and the overall Agatston calcium score did not differ between the groups. However, a significant number of patients with ET had a high calcium score not predicted by the Framingham risk equation for coronary disease. While a significant correlation between the Framingham CHD risk and the Agatston was found for control subjects $(\mathrm{r}=0.577, \mathrm{p}<0.001)$, no significant correlation was found fort ET patients $(\mathrm{r}=0.197, \mathrm{p}=0.223)$.

Significantly more patients with JAK2 positive ET than control subjects had a coronary calcium Agatston score of $>160$, indicating high cardiovascular risk, not predicted by the Framingham equation. CT calcium score is a robust, available, simple test which might identify ET patients at high risk for coronary events.

References: Hasselbalch HC. Perspectives on chronic inflammation in essential thrombocythemia, polycythemia vera, and myelofibrosis: is chronic inflammation a trigger and driver of clonal evolution and development of accel. atherosclerosis...Blood 2012; 119: 3219-25., Celermajer DS. Reliable endothelial function testing: at our fingertips? Circulation 2008, 117: 2428-30., Hamburg NM, Keyes MJ, Larson MG, Vasan RS, Schnabel R, Pryde MM, et al. Cross-sectional relations of digital vascular function to cardiovascular risk factors in the Framingham Heart Study. Circulation 2008; 117: 2467-74., Arad Y, Spadaro LA, Goodman K, NewsteinD, Guerci AD. Prediction of coronary events with electron beam computed tomography. J Am Col Cardiol 2000; 36: 1253-60.

Keywords: arterial wall; functional properties; morphological properties; calcium score; Framingham risk score; myeloproliferative disease

\section{L-2-hydroxyglutaric aciduria- case report}

Hristina Janeski', Brankica Dimitrijevic ${ }^{1}$, Marija Mijovic ${ }^{1}$, Vesna Dimitrijevic- Sreckovic ${ }^{2}$, Goran Cuturilo ${ }^{1}$

1. Clinical genetics, University Children's Hospital, Belgrade, Serbia

2. Internal medicine, Clinic for endocrinology, diabetes and metabolic diseases, Belgrade, Serbia

Background: L-2-Hydroxyglutaric (L-2-HG) aciduria is a rare inherited metabolic disease usually seen in children and young adults. Symptoms and signs includes slowly progressive deterioration with cerebellar ataxia, dysartria, macrocephaly, mild or severe mental retardation, seizures, extrapyramidal and pyramidal symptoms. MRI studies shows subcortical white matter lessions, followed by cerebellar atrophy and changed structure of basal ganglia. The disease is characterized by increased levels of L-2-HG in urine and cerebrospinal fluid. According to literature this would be the third variant described.

Objective: The aim of this report is to publish this rare homozygous pathogenic variant in L2HGDH gene, and its correlation with increased risk for developing brain tumors.

Methods: Neurologic and pediatric examination found present extrapyramidal and cerebellar signs and symptoms. MRI studies showed multiple subcortical lesions of white matter, also changes in basal ganglia are found. Urin analisys showed increased leves of hydroxyglutaric acid. Molecular genetic testing, sequencing of L2HGDH gene is done.

Result: Sequencing of L2HGDH gene showed rare homozygous pathogenic variant c.530_533delinsATT p. (Pro177Hisfs ${ }^{\star} 6$ ) and confirmed this rare metabolic condition. This result is conclusive with MRI studies and clinical findings in our patient.

Conclusion: L-2-HG aciduria is a rare metabolic disease with slow progressive clinical course. Cerebellar pathology is main clinical finding, confirmed by MRI. In this case we found rare homozygous pathogenic variant in L2HGDH gene, documented in two cases by now. It is important to raise attention to this metabolic disease as L-2HG is a putative oncometabolite and it correlates with high incidence of brain tumors mostly, but also renal and bone tumors are reported, so cancer surveillance is indicated in this group of patients. Therapy for these patients is symptomatic and consists of ribofalvin and L-carnitin, to reduce the symptoms and increase motility and functionality.

References: Wagner S, Vianey-Saban C, Salomons GS, Schmitt E, Feillet F. L-2-hydroxyglutaric aciduria: report on two cases. Arch Pediatr. 2014 Jan;21(1):78-85

Keywords: L-2-hydroxyglutaric aciduria, oncometabolite, rare homozygous variant, L2HGDH gene

\section{Obesity, metabolic syndrome and prediabetes are associated with increased cancer risk}

Vesna Dimitrijevic-Sreckovic ${ }^{1}$, Milica Vujovic ${ }^{2}$, Hristina Janeski ${ }^{3}$, Branko Sreckovic ${ }^{4}$, Nevena Pajovic ${ }^{2}$, Sasa Ilic ${ }^{2}$, Ivan Soldatovic ${ }^{5}$ 1. Diabetology, Clinic for Endocrinology, Diabetes and Metabolic Diseases, Clinical Center of Serbia; Faculty of Medicine, Belgrade University, Serbia, Belgrade, Serbia

2. Clinic for Endocrinology, Diabetes and Metabolic Diseases, Clinical Center of Serbia, Belgrade, Serbia

3. Pediatrics, University Children's Hospital, Belgrade, Serbia

4. Internal Medicine, General Hospital Aurora, Belgrade, Serbia

5. Statistics, Institute for Medical Statistics and Informatics, Faculty of Medicine, Belgrade University, Serbia, Belgrade, Serbia 
Background: Obesity and metabolic syndrome (MS) are followed by insulin resistance (IR) and hyperinsulinism, which in return effects mitogenic signaling pathways contributing to carcinogenesis. MS is associated with atherosclerosis, depression, infertility and cancer.

Objectives: The aim of this study is to examine frequency of certain tumors in obese and overweight patients with glycoregulation disorders, impaired fasting glucose (IFG) and impaired glucose tolerance (IGT). To analyze correlation of MS factors with cancer risk.

Methods: The study included patients, aged over 45 years. Who were overweight-BMI $25-30 \mathrm{~kg} / \mathrm{m} 2$ and obese-BMI $>30 \mathrm{~kg} / \mathrm{m} 2$, classified into three groups: I-obese patients; II-patients with IFG; III-patients with IGT. IDF classification was applied for diagnosing MS. IR was determined by homeostatic model assessment (HOMA IR).

Results: Mean age of patients is $62.7 \pm 6.8$ years, mostly female population $96.3 \%$. Patients with IFG have increased waist circumference (WC), BMI, cholesterol, LDL-cholesterol, triglyceride, diastole blood pressure, microalbuminuria, mean insulin value in OGTT 50.8 $\pm 42,3 \mathrm{mU} / 1$ and creatinine clearance followed by decreased vitamin D levels and HDL- cholesterol compared to the other two groups. IFG patients have increased values of tumor markers for breast cancer Ca 15-3 (74.6 \pm 14.2$)$ and colon cancer Ca 19-9 ((107.4 \pm 241.3$)$. In obese patients most frequent cancers are of the breast 33, 3\%, uterine cervix 22,2\%, uterine endometrial 11,1\%, and ovary $11,1 \%$. In IGT patients most frequent is breast cancer $63,6 \%$, prostate cancer $9,1 \%$ and thyroid cancer $9,1 \%$.

Conclusion: Obesity in menopausal women is associated with increased risk for carcinoma and most frequent is breast cancer. Increased insulin values and decreased vitamin D is present in all three examined groups of patients. MS is mostly frequent in IFG patients with carcinoma where all MS factors are present. Aggravation of glycoregulation is followed by increasing in number of MS factors, so IFG patients usually have four and IGT patients have all five factors, accompanied by increased cancer prevalence. Body weight reduction by Mediterranean diet and physical activity, decreasing of abdominal obesity and hyperinsulinism, correction of hyperproteinergic diet with vitamin $\mathrm{D}$ substitution may contribute in cancer prevention.

References: Scappaticcio L, Maiorino MI, Bellastella G, Giugliano D, Esposito K. Insights into the relationships between diabetes, prediabetes, and cancer. Endocrine. 2017 May;56(2):231-239

Keywords: metabolic syndrome, obesity, cancer, IFG, IGT

\section{Molecular genetic diagnosis of monogenic forms of diabetes in Russia}

Oleg Glotov ${ }^{1}$, Elena Serebryakova ${ }^{1}$, Maria Turkunova ${ }^{2}$, Elena Bashnina ${ }^{3}$, Andrey Glotov ${ }^{1}$, Yuri Barbitov ${ }^{1}$, Tatiana Ivaschenko ${ }^{4}$, Mikhail Fedyakov $^{1}$, Andrey Sarana ${ }^{1}$, Sergey Scherbak ${ }^{1}$, Vladislav Baranov ${ }^{1}$

1. Institution of Translation medicine, Saint Petersburg State University, St-Petersburg, Russian Federation

2. St. Petersburg state pediatric medical University, Ministry of health of Russia, St-Petersburg, Russian Federation

3. North-Western State Medical University named after I.I Mechnikov, Ministry of health of Russia, St-Petersburg, Russian Federation

4. The Research Institute of Obstetrics, Gynecology and Reproductology named after D.O.Ott, St-Petersburg, Russian Federation

The aim of the study was to determine the detection and spectrum of mutations in children with MODY in Russia.

Methods and materials. The study included patients with diabetes manifested at the age of the first 6 months of life, as well as a group of patients with "mild" diabetes course preserved insulin secretion, the absence of diabetogenic autoantibodies. By full-exom sequencing study we are analyzed the coding regions of genes which are associated with MODY: HNF1A, GCK, HNF4A, HNF1B, PDX1, NEUROD1, KLF11, CEL, PAX4, INS, BLK, EIF2AK3, RFX6, WFS1, ZFP57, FOXP3, KCNJ11, ABCC8, GLUD1, HADH (SCHAD), SLC16A1, UCP2, INSR, AKT2, GCG GCGR, PPARG, PTF1A. Bioinformatic filtering of DNA sample sequencing results was performed using the following programs: "GeneTalk" (https:// www.gene-talk.de/), “UGENE” (http://ugene.unipro.ru/), “Ion Reporter” (https://ionreporter.lifetechnologies.com/ir/), "SIFT” (http://sift. jcvi.org/), "PolyPhen2” (genetics.bwh.harvard.edu/pph2/), "PAPI” (http://papi.unipv.it/). The original previously developed metric was used for ranking the variants. Verification of the obtained data was carried out by direct sequencing on the device "Genetic Analyzer 3500" (“Applied Biosystems”, USA).

Results: DNA samples of 72 patients with suspected MODY were examined. MODY was confirmed in 51\% ( $\mathrm{n}=37)$. The most common mutations in the GCK gene were 39\% $(n=23), 3$ patients had pathogenic mutations in several genes 4\%, HNF1A $-2.7 \%(n=2), W F S 1-2.7 \%(n=2)$, EIF2AK3-2.7\% ( $n=2)$, PAX4-1.4\% ( $n=1)$, GATA6-1.4\% $(n=1)$, FOXP3-1.4\% $(n=1), \operatorname{KCNJ11-1.4\% ).~}(n=1)$ ABCC8-1,4\% $(n=1)$.

Conclusion: the frequency of detection of MODY in our group is higher than described in the literature, and is $51 \%$. High detectability can be associated with both the characteristics of our group and the bioinformatic approach used. Molecular genetic verification of the diagnosis by NGS sequencing allows to predict the course of the disease and make adjustments in the treatment of diabetes.

Research supported by the framework “Alfa-endo” and grant RSF №14-50-00069, sequencing performed in "Biobank Center” of the Science Park of SPSU.

\section{APOE genotypes in Lebanon: Distribution and association with hypercholesterolemia and Alzheimer's disease}

Said El Shamieh ${ }^{1}$, Christy Costanian², Rayan Kassir ${ }^{3}$, Sophie Visvikis Siest ${ }^{4}$, Nisrine Bissar ${ }^{3}$

1. Medical Laboratory Sciences, Beirut Arab University, Beirut, Lebanon

2. School of Kinesiology and Health Science, York University, Toronto, Canada

3. Beirut Arab University, Beirut Arab University, Beirut, Lebanon.

4. UMR INSERM U1122; IGE-PCV, University of Lorraine, Nancy, France 
We first investigated the distribution of APOE genotypes in Lebanese general population (LGP) and Alzheimer's disease study (ADS) groups, and compared it with 1000genomes populations. Then, we assessed eventual association between APOE genotypes, hypercholesterolemia and AD. This cross-sectional study was conducted on 591 individuals. Prevalence of APOE genotypes in Lebanon was similar to that seen in Asian populations. APOE genotypes were not associated with hypercholesterolemia. A significant difference between APOE genotypes in $\mathrm{AD}$ cases versus controls and versus LGP was seen. Moreover, E4 allele was $\sim 3$ fold higher in ADS patients when compared to the remaining individuals. We established the distribution of APOE genotypes in the Lebanon and showed that in contrast to lipid profile, APOE4 correlates with AD.

\section{Hellp syndrome}

Affaf ADDA ${ }^{1}$, Mohamed CHEKKAL 2 , Nazim BENNAOUM² ${ }^{2}$ Mohammed HAMMADI ${ }^{2}$

1. Hemobiology and transfusion medicine, University hospital "EHU 1 er Novembre 1954", Oran, Algeria

2. University hospital "EHU 1 er Novembre 1954”, Oran, Algeria

HELLP syndrome is a thrombotic microangiopathy, considered a particular clinical form of preeclampsia (or toxemia of pregnancy). Often insidious with non-specific clinical signs, it is grafted with a heavy maternal morbidity.

According to the physiopathology of hellp syndrome, schizocytes are a constant component.

Our work focuses on schizocyte research and its interest in diagnosing hellp syndrome. However, their highlighting remains difficult and this amounts to the variability of the identification criteria due to a lack of standardization that has been known for a long time.

The aim of our work is to study the relationship of schizocytes with hellp syndrome in 12 parturients with a hellp syndrome, focusing on the influence of this disease on their schizocyte rate. We will also study the relationship of schizocytes with the different major criteria involved in hellp syndrome (platelets and hemoglobin).

Keywords: hellp syndrome, preeclampsia, toxemia

\section{Uncovering Clinically Relevant Mutations in Membrane Transporters by Genetic Analysis Linked to the Determination of Erythrocyte Membrane Protein Expression}

Balazs Sarkadi

Biomembrane, Institute of Enzymology, Research Centre for Natural Sciences, Hungarian Academy of Sciences, Budapest, HUNGARY

Introduction: The human red blood cell (RBC) membrane contains more than 300 integral membrane proteins, many with high relevance to disease conditions or pharmacological interventions.

Objectives: We have developed a flow cytometry method for the quantitative determination of the RBC membrane expression levels of selected membrane transporters, and examined their molecular genetic background, as well as their potential value in medical diagnostics as biomarkers.

Methods: We have analyzed the expression of the ABCG2 xeno- and endobiotic transporter, modulating the absorption and metabolism of pharmacological agents and causing multidrug resistance in cancer. ABCG2 is also involved in uric acid elimination and its impaired function is causative in gout.

Results: Analysis of ABCG2 expression in the erythrocyte membranes of healthy volunteers and gout patients showed an enrichment of lower expression levels in the gout patients, correlating with a genetic polymorphism, causing a Q141K variant protein. In addition, based on RBC expression levels, we found stop and frameshift mutations, as well as a relatively frequent, novel ABCG2 mutation (ABCG2-M71V). This variant, according to cellular expression studies, causes reduced protein expression, although with preserved transporter capability. ABCG2-M71V expression in vitro could be corrected by therapeutically relevant small molecules, with a potential in allele-specific gout treatment. Conclusions: These results suggest that personalized medicine should consider the genetic analysis linked to RBC membrane protein expression, and these studies may provide a new tool to uncover clinically important variants of membrane proteins.

Supported by OTKA K115375, K111678 and FIEK 16-1-2016-0005, NKTH, grants.

Keywords: ABCG2 multidrug transporter, gout, erythrocyte membrane

\section{Association of the genotype and metabolic profile of athletes in the formation of adaptations to physical activity}

D. Alaverdian $^{1,3}$, O. Glotov ${ }^{1,2,3}$, O. Mamaeva ${ }^{1}$, S. Namozova ${ }^{3}$, D. Leshchev ${ }^{1,4}$, M. Fedyakov ${ }^{1,3}$, N. Shved ${ }^{1,2,3}$, M. Aseev ${ }^{1,2}$, I. Poliakova ${ }^{1,3}$, V. Merkureva ${ }^{3}$, A. Kergaard ${ }^{5}$, S. Shcherbak ${ }^{1,3}$, A. Sarana ${ }^{1,3}$, S. Urazov ${ }^{1}$, V. Smirnov ${ }^{1}$, S. Makarenko ${ }^{1}$

1 City hospital N40, Borisova street, 9, Sestroretsk, Saint Petersburg, Russian Federation

2 D. O. Ott Research Institute of Obstetrics and Gynecology, Mendeleevskaya line, 3, Saint Petersburg, Russian Federation

3 Saint-Petersburg State University, Universitetskaya emb., 7/9, Saint Petersburg, Russian Federation

4 Peter The Great Saint Petersburg Polytechnic University, Polytechnicheskaya, 29, Saint Petersburg, Russian Federation

5 Lesgaft National State University of Physical Education, Sport and Health, Dekabristov street, 35, Saint Petersburg, Russian Federation 
Introduction: Adaptation to physical stress caused by regular trainings as well as the level of expression of many genes, which may change depending on nucleotide substitutions in the coding regions. It is known that there are different variants of genes that influence key functions of energy metabolism. To study the mechanisms of adaptation to physical loads we selected genetic markers which can have a direct or indirect influence on the change of metabolism during the trainings.

Methods: The study involved 78 athletes of various specializations and training levels. Clinical and instrumental studies were carried to evaluate the cardiovascular system and the BRUCE stress test with fixation of blood pressure and heart rate.

The sample group of athletes was divided into the following groups:

High and low levels of metabolic equivalent (MET) values based on the treadmill test [1].

Regarding the type of exercise performed (three groups). Each sport, related to both dynamic and static loads, can be classified as low-, medium- or high-intensity. Using PCR-RFLP analysis identified genotypes of: ACE (rs4340), PPARA (rs2016520), PPARD (rs2016520), PPARG (rs1801282), and PPARGC1A (rs8192678), AMPD1 (rs17602729), ACTN3 (rs1815739), DRD2A (rs1800497), HTR2A (rs6313).

Results: Confirmed and complemented by the functional association of genes PPARGC1A and ACE with physiological and biochemical parameters. Was found a correlation between variants of the HTR2A gene and a view of the executed workload. We obtained significant differences in the frequency of occurrence of polymorphic variants of the gene of the serotonin receptor HTR2A. The T/T genotype was met more often in the group, performing highly dynamic loads (45\%) than in the other groups (13\% and $18 \%, \mathrm{P}=0.03)$.

We confirmed the functional association of the $A C E$ gene with the level of blood pressure: the D/D genotype causes a higher blood pressure. In addition, it should be mentioned that the $\mathrm{D} / \mathrm{D}$ genotype is associated with an increased risk of cardiovascular disease, which can be especially dangerous for people engaged in sports.

Conclusion: In this research we analyzed the association of clinical and instrumental studies with different alleles of one of the most important metabolic genes in people who are regularly exposed to physical stress. Founded functional connections of the key genes of energy metabolism with biochemical blood values, which confirm and supplement the literature data, show us that these polymorphisms do play a role in adaptation to physical activities and influence the level of gene expression.

Reference: 1. Tavrovskaya T.V. Bicycle ergometry. // Manual for doctors. St. Petersburg. 2007. P. 134.

Keywords: sport genetics, metabolism genes, polymorphism, adaptation, treadmill test.

[Correction added after online publication 9 November 2018: Information about co-authors and affiliations was added. In the version that was originally published, only Diana Alaverdian was listed.]

\section{Database-guided flow-cytometry for evaluation of dysplasia on bone marrow myeloid cells in Myelodysplastic Syndromes}

Denis Guyotat ${ }^{1}$, Carmen Mariana Aanei², Marie-Christine Jacob³ ${ }^{3}$, Richard Veyrat-Masson ${ }^{4}$, Maria-Alessandra Rossenthal-Allieri ${ }^{5}$, AnneCatherine Lhoumeau ${ }^{6}$, Michel Ticchioni ${ }^{5}$, Florent Dumezy ${ }^{7}$, Lydia Campos Catafal ${ }^{2}$

1. Departement d'Hématologie et Thérapie Cellulaire, Institut de Cancérologie Lucien Neuwirth, Saint Priest en Jarez, France

2. Laboratoire d'Hématologie, CHU de Saint-Etienne, Saint-Etienne, France

3. Laboratoire d'Immunologie, CHU de Grenoble, Grenoble, France

4. Laboratoire d'Hématologie, CHU de Clermont-Ferrand, Clermont-Ferrand, France

5. Laboratoire d'Immunologie, CHU de Nice, Nice, France

6. Hématologie, Institut Paoli-Calmette, Marseille, France

7. Laboratoire d'Hématologie, CHU de Lille, Lille, France

Introduction: Myelodysplastic syndromes (MDS) are clonal hematopoietic stem cells disorders driven by spliceosome mutations cooperating with specific epigenetic modifiers. The resulting phenotype is characterized by morphological and antigen expression differentiation anomalies. However the large heterogeneity of MDS still makes their diagnosis a challenge, particularly in cases with minimal morphological dysplasia and absence of cytogenetic anomalies. Despite international efforts to implement multicolor flow-cytometry (MFC), considerable variability remains in data interpretation. The aim of this study was to evaluate the utility of MFC analysis for MDS diagnosis when patient data are compared against maturation databases built from normal bone marrow (BM) samples.

Methods: A working group of 6 laboratories was initiated to standardize MFC procedures for MDS diagnosis. Sample preparation and marker combinations were performed according to Euroflow recommendations. Three maturation databases were developed for BM myeloid lineages using data acquired in BM aspirates from 18 healthy donors. Samples from patients with suspicion of MDS were analyzed similarly and compared to normal maturation databases. The phenotypic changes $>2 \mathrm{SD}$ compared to the median of the "normal BM" group were considered abnormal. Results: The building of databases allowed identifying and fixing several issues related to sample preparation and acquisition. The most common staining issues were for CD11b and CD13 in neutrophils, CD300e and HLADR in monocytes, CD71 and HLADR in erythroid cells.

The comparison of samples from patients with suspicion of MDS allowed the identification of phenotypic anomalies in early stage MDS, in immature CD34+ and/ or CD117+ precursors (e.g. diminution of expression of CD34, CD117 and HLADR on neutrophil immature precursors; increased expression of CD35 and early acquisition of CD300e on monocytic precursors; decreased expression of CD36 on early erythroid precursors) and in mature myeloid cells that are difficult or even impossible to detect using current analysis strategies.

Conclusion: The building of reference databases incorporating flow cytometry data from normal subjects contributes to a more objective, standardized analysis of pathologic samples. 


\title{
Characterization of single nucleotide polymorphisms associated with response to antidepressant drugs in Sudanese
}

\author{
Suaad Suaad Balla', Mohamed Mohamed O Elsiddeig', Muntaser Muntaser Eltyeb Ibrahim² \\ 1. Institute of endemic disease, Khartoum, Sudan \\ 2. Molecular biology, Institute of endemic disease, Khartoum, Sudan
}

Background: Tricyclic antidepressants and SSRIs, prescribed for major depressive disorders are associated with therapeutic failures in approximately $40 \%$ of patients after initial dosing. Pharmacogenetic (PGx) variances play a significant role in these failures and therefore, using genetic data in decision-making for population based and personalized dosing may both enhance efficacy and reduce adverse effects. Prior knowledge of allele distributions of the (PGx) biomarkers in different countries can help towards patient stratification for most populations during the drug prescribing process. However, Allele frequency distributions for gene polymorphisms associated with Antidepressant response in the Sudanese population and Africa in general remains largely non characterized.

Objective: To investigate single nucleotide Polymorphisms [SNPs] affecting several pharmacodynamic and pharmacokinetic variables associated with response to antidepressant drugs [AD] in Sudanese populations and to compare the data with the global geographic data reported in Gnomad from different ethnic populations

Design: A total of 71 SNPs in 7 candidate genes (1) affecting response to (AD) in 221 healthy Sudanese individuals were analyzed. Genotype data are chosen according to extensive reviews, deleteriousness and clinically actionable variants reported in PharmGKB database. Allele frequencies of these variants in Sudanese are reported in comparison to global frequencies.Result: Twelve unreported SNPs in gnomad genome database were found in Sudanese, 11 of which had MAF < 1\% and 1 SNP (rs10276036) in ABCB1 gene with frequency of 20\%. 2 SNPs (rs7103411) and ( rs7124442) in BDNF gene associated with better response to citalopram is less common in Sudanese population compared with other population.

Conclusion: Since therapeutic decision and recommendations rely mainly on the US FDA or European Medical Agency guidelines for dosing instructions, a comprehensive understanding of the pharamacogenetic inter-ethnic differences like in the Sudanese, is critical to guide more effective individualized and population tailored prescriptions, such data will be useful for future clinical and for drug dosage recommendations population

References: Reyes-Barron C, Tonarelli S, Delozier A, Briones DF, Su BB, et al. (2016) Pharmacogenetics of Antidepressants, A Review of Significant Genetic Variants in Different Populations. Clin Depress 2:109

Keywords: Antidepressant drug, SNP, pharmacogenetics,

\section{Novel insights into the function and implication of an autophagy-focal adhesion signaling loop involved in triple negative breast cancer progression}

Moulay Alaoui-Jamali, Chia Hao, Krikor Bijian, Jie Su, Dominik Wernic

Segal cancer Center and Lady Davis Institute for Medical Research, Departments of Medicine and Oncology, Faculty of Medicine, McGill University, Montreal, Qc. H3T 1E2, Canada

Introduction: Among breast cancer subtypes, triple negative breast cancer (TNBC, negative for estrogen, progesterone and Her-2 receptors) has a high incidence of metastasis and lacks targeted therapeutics, in contrast to estrogen- and Her2-positive subtypes. A critical early step for TNBC invasion is the excessive remodeling of local tumor microenvironment and acquisition of cell motile properties in response to chemotactic signals. Motile cancer cells exhibit finger-like plasma membrane protrusions that are stabilized by focal adhesions (FAs). The later are dynamic protein complexes formed near leading edge sites of cell protrusions to couple cell matrix receptors to the actin cytoskeleton. Synchronous assembly/disassembly of FAs at these sites is essential for the generation of traction forces necessary for cell locomotion. The regulation and clinical implication of these processes in cancer remain partially understood.

Objectives: The objective of this study is the identification of rate-limiting step molecular targets that control early TNBC cell dissemination and can be exploited to develop promising selective therapeutics.

Methods: We used a combination of high-resolution imaging studies coupled to biochemical and molecular gene manipulation tools to address FA dynamics in relation to cell locomotion using isolated TNBC cells and preclinical TNBC in vivo models. Comparative genomic hybridization and genome-wide screening were used to establish the relevance of autophagy-FA regulators to prognosis in breast cancer cases from patients with progressive disease.

Results: We demonstrated that autophagy promotes activation of FA turnover at plasma membrane protrusions. Inhibition of autophagy using genetic approach or pharmacological inhibitors prevented FA disassembly (but not assembly) and inhibited cancer cell migration in vitro. FA disassembly occurred in part via recruitment of paxillin, a FA adapter and signaling protein, to autophagosomes via the autophagy cargo protein c-Cbl. Analysis of phosphorylated and phosphor-mutant forms of paxillin revealed recruitment of this protein to autophagosomes to require phosphorylation at specific tyrosine residues. In orthotopic TNBC mouse xenograft models coupled to tissue immunohistochemistry we observed that co-targeting of FA and autophagy induced a potent anti-metastasis activity. Finally, genome-wide screening of a large cohort of cancer tissues from patients revealed co-overexpression of specific FA and autophagy regulators to predict progression and poor prognosis. Conclusion: These results provide insights into molecular pathways that control TNBC invasiveness and support the potential therapeutic utility of targeting the FA-autophagy axis.

Acknowledgements: Supported by the QBCF and the CIHR. 


\title{
LSR and APOE interactions and their role in personalised medicine of type III hyperlipidemia
}

\author{
MG Stathopoulou ${ }^{1}$, T Xie ${ }^{1}$, S Akbar ${ }^{1,2}$, T Oster ${ }^{2}$, FT Yen ${ }^{2}$, S Visvikis-Siest ${ }^{1,3}$ \\ 1 Université de Lorraine, Inserm, IGE-PCV, Nancy, France \\ 2 EA3998 INRA USC 0340 UR AFPA, Université de Lorraine, Vandoeuvre-lès-Nancy, France \\ 3 Department of Internal Medicine and Geriatrics, CHU Nancy-Brabois, Nancy, France
}

Background: The lipolysis stimulated lipoprotein receptor (LSR) is an apolipoprotein (Apo) B and ApoE receptor that participates in the removal of triglyceride-rich lipoproteins during the postprandial phase. LSR gene is located upstream of APOE, an important risk factor for cardiovascular disease (CVD).

Objective: This project study aims to determine the potential impact of functional SNPs of $L S R$ and APOE gene in the development of cardiovascular diseases and hypelipidemias such as type III hyperlipidemia. In an initial project we have focused on the effect of the SNP rs916147 in LSR gene on lipid traits in healthy subjects and we have investigated potential epistatic interaction between $L S R$ and APOE.

Design: Unrelated healthy adults $(n=432)$ and children $(n=328,<18$ years old) from the STANISLAS Family Study were used.

Results: Age-specific epistasis was observed between $A P O E$ and $L S R$, reversing the protective effect of APOE $\varepsilon 2$ allele on cholesterol, ApoE and low-density lipoprotein levels ( $\beta$ : .114, P: .777 $\times 10^{-8}, \beta: .125$, P: .639 $\times 10^{-3}, \beta: .059$, P: .531 $\times 10^{-3}$, respectively). This interaction was verified in an independent adult population $(\mathrm{n}=1744)$.

Conclusions: These results highlight the importance of the LSR polymorphism and reveal the existence of complex molecular links between LSR and ApoE for the regulation of lipid levels, revealing potential new pathways of interest in type III hyperlipidemia and its involvement in CVD pathology. These pathways are assessed through epidemiological and functional studies (in vitro and in vivo) in ongoing projects that will lead to the understanding of the role of LSR in type III hyperlipidemia and provide a new tool for personalised medicine in this field.

Acknowledgments: The study is funded by the "Région Lorraine" and cofinanced by the European Union within the framework of the Operational Program FEDER Lorraine et Massif des Vosges 2014-2020.

References: Xie T, Stathopoulou MG, Akbar S, Oster T, Siest G, Yen FT, Visvikis-Siest S. Effect of LSR polymorphism on blood lipid levels and age-specific epistatic interaction with the APOE common polymorphism. Clin Genet. 2018;93(4):846-852.

\section{Telomere length in personalised medicine}

V Gorenjak $^{1}$, MG Stathopoulou ${ }^{1}$, AM Petrelis ${ }^{1}$, S Visvikis-Siest ${ }^{1,2}$

1 Université de Lorraine, Inserm, IGE-PCV, Nancy, France

2 Geriatric Service, Nancy University Hospital, Nancy, France

Background: Telomere length has been the subject of studies for many decades, aiming to elucidate its role in physiological processes, in the process of aging and in diverse pathologies. Yet today, there is still no "big title" discovery that would lead to a practical use of telomeres as a reliable biomarker or target for a new drug. However, therapies for chronic disease patients are being tested and companies are already offering commercial tests for telomere length measurement. The strong genetic heritability of telomeres is opening the place for pharmacogenomics researches that could promote the personalized treatment of diverse diseases.

Objective: In the unit U1122 we are focused on the identification of telomere length determinants of children and adults in health and disease. We have been based on our preliminary results on children.

Design: Healthy children $(\mathrm{n}=322$, age range $=6.75-17$ years) with available GWAS data (Illumina Human CNV370-Duo array) were included. The LTL was measured using multiplex quantitative real-time polymerase chain reaction. Linear regression models adjusted for age, gender, parental age at child's birth, and body mass index were used to test the associations of LTL with polymorphisms identified in adult GWAS and to perform a discovery-only GWAS.

Results: The previously GWAS-identified variants in adults were not associated with LTL in our paediatric sample. This lack of association was not due to possible interactions with age or gene $\times$ gene interactions. Furthermore, a discovery-only GWAS approach demonstrated six novel variants that reached the level of suggestive association $(\mathrm{P} \leq 5 \times 10(-5))$ and explain a high percentage of children's LTL.

Conclusions: The study of genetic determinants of LTL in children may identify novel variants not previously identified in adults. Studies in large-scale children populations are needed for the confirmation of these results, possibly through a childhood consortium that could better handle the methodological challenges of LTL genetic epidemiology field. There are ongoing studies currently developed in the U1122 unit and an extended network of collaborators on telomere length genetic determinants in different age groups and diseases and on the role of telomere length as a biomarker in personalised medicine.

Acknowledgments: This work is supported partly by the french PIA project «Lorraine Université d'Excellence», reference ANR-15-IDEX-04-LUE. References: Stathopoulou MG, Petrelis AM, Buxton JL, Froguel P, Blakemore AI, Visvikis-Siest S. Genetic determinants of leucocyte telomere length in children: a neglected and challenging field. Paediatr Perinat Epidemiol. 2015;29(2):146-50. 


\section{The VEGF Consortium}

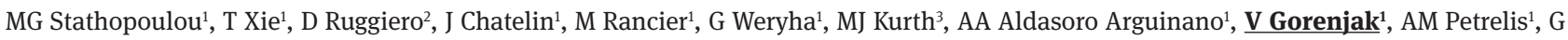
Dagher $^{4}$, G Dedoussis ${ }^{5}$, P Deloukas ${ }^{6}$, L Lamont ${ }^{3}$, J Marc ${ }^{7}$, M Simmaco ${ }^{8}$, RHN Van Schaik ${ }^{9}$, F Innocenti ${ }^{10}$, JL Merlin ${ }^{11}$, J Schneider ${ }^{12}$, BZ Alizadeh ${ }^{13}$, M Ciullo² $^{2}$ S Seshadri ${ }^{14}$, S Visvikis-Siest' ${ }^{1}$; VEGF Consortium.

1 Université de Lorraine, Inserm, IGE-PCV, Nancy, France.

2 Institute of Genetics and Biophysics, National Research Council of Italy, Naples, Italy.

3 Randox Laboratories Ltd., Crumlin, UK.

4 Biobanking and Biomolecular Resources Research Infrastructure (BBMRI)/INSERM US 13, BIOBANQUES, Paris, France.

5 Department of Nutrition Dietetics, Harokopio University of Athens, Athens, Greece.

6 Queen Mary University of London, London, UK.

7 Faculty of Pharmacy, University of Ljubljana, Ljubljana, Slovenia.

8 Sant'Andrea Hospital - Sapienza University of Rome, Rome, Italy.

9 European Society of Pharmacogenomics and Personalised Therapy (ESPT), Nancy, France.

10 University of North Carolina at Chapel Hill, Chapel Hill, NC, USA.

11 Institut de Cancérologie de Lorraine et Université de Lorraine, Vandoeuvre les Nancy, France.

12 University of Luxembourg, Luxembourg, Europe.

13 Department of Epidemiology, University of Groningen, University Medical Center Groningen, Groningen, The Netherlands.

14 Department of Neurology, Boston University School of Medicine, Boston, MA, USA.

Background: The Vascular Endothelial Growth Factor (VEGF) is a key molecule of angiogenesis with an important impact both on physiological processes and on the development of complex chronic diseases. The Vascular Endothelial Growth Factor European Genomic Federation - VEGF Consortium (http://www.vegfconsortium.org) aims to develop a transnational collaborative network dedicated to large integrative and multidisciplinary genomic studies on VEGF in order to generate actionable knowledge for medical practice of chronic diseases.

Objective: To identify VEGF '-omics' profiling in health and disease to be used for patient stratification.

Design: Fourteen partners from 8 countries are involved in the consortium, representing ten internationally recognized cohorts totaling $>15000$ individuals. These partners are among the worldwide leaders in the fields of genomics, transcriptomics, epigenomics, “-omics” methodologies and analyses, chronic diseases prediction and prevention, personalised medicine, personalised therapy, pharmacogenomics, clinical implementation, and communication.

Results: Through two Genome-Wide Association Studies, we have identified 10 genetic variants, collectively explaining up to 50\% of VEGF phenotypic variation. We have also demonstrated associations between these polymorphisms and cardiovascular (CVD) risk factors such as blood circulating lipid levels, depression, autoimmune thyroid diseases. Eleven working groups have been formed.

Conclusions: The VEGF consortium brings together a large number of high-level researchers and academics, along with their expertise and resources. It is expected to provide robust results on the role of VEGF in chronic diseases prediction, prevention and personalized therapy. It is an original concept open to further collaborations in order to advance research on this challenging field.

Acknowledgments: The study was funded by the "Région Lorraine" and cofinanced by the European Union within the framework of the Operational Program FEDER Lorraine et Massif des Vosges 2014-2020.

References: Stathopoulou MG, Xie T, Ruggiero D, Chatelin J, Rancier M, Weryha G, Kurth MJ, Aldasoro Arguinano AA, Gorenjak V, Petrelis AM, Dagher G, Dedoussis G, Deloukas P, Lamont J, Marc J, Simmaco M, Schaik RHNV, Innocenti F, Merlin JL, Schneider J, Alizadeh BZ, Ciullo M, Seshadri S, Visvikis-Siest S; VEGF Consortium. A transnational collaborative network dedicated to the study and applications of the vascular endothelial growth factor-A in medical practice: the VEGF Consortium. Clin Chem Lab Med. 2018;56(4):83-86.

\section{TREM-1 variants associations with TREM-1 protein and mRNA levels and adhesion molecules}

AA Aldasoro Arguinano ${ }^{1}, \mathrm{~S}$ Dadé ${ }^{1}$, MG Stathopoulou ${ }^{1}$, M Derive $^{2}$, NC Ndiaye ${ }^{1}$, T Xie $^{1}$, C Masson $^{1}$, S Gibot $^{3}$, S Visvikis-Siest $^{1,4}$

1 Université de Lorraine, Inserm, IGE-PCV, Nancy, France

2 INOTREM, Nancy, France.

3 Réanimation Médicale, Hôpital Central, Nancy, France.

4 Department of Internal Medicine and Geriatrics, CHU Technopôle Nancy-Brabois, Rue du Morvan, Vandoeuvre-lès-Nancy, France.

Background: High levels of TREM-1 are associated with cardiovascular and inflammatory diseases risks and the most recent studies have showed that TREM-1 deletion or blockade is associated with up to $60 \%$ reduction of the development of atherosclerosis. So far, it is unknown whether the levels of TREM-1 protein are genetically regulated. Moreover, TREM family receptors have been suggested to regulate the cellular adhesion process.

Objective: The goal of this study was to investigate whether polymorphisms within TREM-1 are regulating the variants of serum TREM-1 levels and the expression levels of their mRNA. Furthermore, we aimed to point out associations between polymorphisms on TREM-1 and blood levels of selectins. 
Design: Thirty unrelated individuals from the STANISLAS Family Study (SFS) were used as discovery cohort for the selection of the TREM-1 SNPs and for their relation with TREM-1 levels. A population of 351 unrelated individuals was used as discovery cohort for the associations with the adhesion molecules levels. An independent population $(n=80)$, composed of unrelated adults of French origin was used as replication population for the results of associations of SNPs with TREM-1 and adhesion molecules levels.

Results: Among the 10 SNPs studied, the minor allele T of rs2234246, was associated with increased sTREM-1 in the discovery population ( $p$-value $=0.003$ ), explaining $33 \%$ of its variance, and with increased levels of mRNA ( $p$-value $=0.007$ ). The same allele was associated with increased soluble L-selectin levels ( $\mathrm{p}$-value $=0.011$ ). The higher levels of sTREM-1 and L-selectin were confirmed in the replication population ( $\mathrm{p}$-value $=0.0007$ and $\mathrm{p}$-value $=0.018$ respectively).

Conclusions: We demonstrated for the first time one SNP on TREM-1, affecting its expression levels. These novel results, support the hypothesis that TREM-1 affects monocytes extravasation and accumulation processes leading to atherogenesis and atherosclerotic plaque progression, possibly through increased inflammation and subsequent higher expression of sL-selectin.

Acknowledgments: This work was supported by the "Agence Nationale de la Recherche, programme d'Investissements d'avenir", grant number ANR-15RHU-0004.

References: Aldasoro Arguinano AA, Dadé S, Stathopoulou M, Derive M, Coumba Ndiaye N, Xie T, Masson C, Gibot S, Visvikis-Siest S. TREM-1 SNP rs2234246 regulates TREM-1 protein and mRNA levels and is associated with plasma levels of L-selectin. PLoS One. $2017 ; 12(8): e 0182226$.

\section{Associations of polymorphisms in the region of the TREM2 gene with TNF- $\alpha$ levels and IMT-F}

V Gorenjak ${ }^{1}$, AA Aldasoro Arguinano ${ }^{1}$, S Dadé $^{1}$, MG Stathopoulou $^{1}$, D Vance ${ }^{2}$, C Masson $^{1}$, S Visvikis-Siest ${ }^{1,3}$

1 Université de Lorraine, Inserm, IGE-PCV, Nancy, France

2 Randox Laboratories Limited, Crumlin, UK

3 Department of Internal Medicine and Geriatrics, CHU Nancy-Brabois, Nancy, France

Background: Triggering receptor expressed on myeloid cells 2 (TREM2) is known for its anti-inflammatory properties during the immune response. Moreover, TREM2 levels have been observed to affect plasma levels of TNF- $\alpha$ and plaque stability in symptomatic and asymptomatic patients with carotid stenosis.

Objective: In this study, we investigated polymorphisms located in the TREM2 gene region and their association with TNF- $\alpha$ levels and the intima media thickness of the femoral artery in healthy individuals.

Design: Discovery population consisted of 139 healthy children from the STANISLAS Family Study (SFS). Five readily available SNPs located in TREM-2 region (rs7748777, rs6918289, rs7759295, rs9357347 and rs6915083 available in the genome-wide scan IlluminaHuman CNV370-Duo array) were analyzed for associations with TNF- $\alpha$ levels, using the R package GWAF (Genome-Wide Association/Interaction Analysis and Rare Variant analysis with Family Data). After performing initial association analysis, the polymorphism rs6918289 was de novo genotyped in 393 adults and 277 more children of SFS and in an independent replication population ( $\mathrm{n}=916)$. De novo genotyping of rs6918289 was conducted by Laboratory of the Government Chemist (LGC) using a PCR-based KASP assay. The association of the rs6918289 with TNF- $\alpha$ plasma levels was assessed separately in the total individuals of SFS (children and adults combined; $\mathrm{n}=809$ ) and in the replication population ( $\mathrm{n}=916$ ). A sub-group of 350 individuals, consisting of adults and children from the SFS population was used to identify associations between intima media thickness of the femoral artery (IMT-F) and rs6918289.

Results: Our results suggest that the minor allele (T) of the rs6918289 is positively associated with elevated plasma levels of TNF- $\alpha$ in the discovery population $(P=0.0026, \beta=0.13, \mathrm{SE}=0.04)$. Similar results were found in the replication population $(\mathrm{P}=0.023, \beta=0.202, \mathrm{SE}=0.09)$. Additionally, rs6918289 was associated with IMT-F in the discovery cohort $(\mathrm{P}=0.026, \beta=0.02, \mathrm{SE}=0.009)$.

Conclusions: These results indicate that rs6918289 may be considered as a risk factor for inflammatory diseases and could be used in personalized medicine for patients diagnosed with chronic inflammatory-related conditions such as atherosclerosis.

Acknowledgments: This work was supported by the "Agence Nationale de la Recherche, programme d'Investissements d'avenir", grant number ANR-15RHU-0004.

References: Gorenjak V, Aldasoro Arguinano AA, Dadé S, Stathopoulou MG, Vance DR, Masson C, Visvikis-Siest S. The polymorphism rs6918289 located in the downstream region of the TREM2 gene is associated with TNF- $\alpha$ levels and IMT-F. Sci Rep. 2018 May 8;8(1):7160.

\section{A case of Constitutional mismatch Repair Deficiency Syndrome caused by PMS1}

$\underline{\text { Reem Salah }}^{1}$, Rayan Seifeldin ${ }^{1}$, Aymen Hussein ${ }^{1}$, Imad Fadl-Almula ${ }^{2}$, Muntaser E Ibrahim ${ }^{1}$

1. Molecular biology department, Institute of Endemic Diseases, University of Khartoum, Khartoum 1111, Sudan.

2. Faculty of Medicine, El-Neeleen University, Khartoum 1111, Sudan

Background: Constitutional mismatch repair deficiency syndrome (CMMRDs) is a devastating cancer predisposition disease for which clinical manifestations, genetic screening, and cancer prevention strategies are limited.

Objectives: To identify and prioritize candidate causative genetic variants that could cause the underlying multiple pediatric cancer in a Sudanese family. 
Subjects and Methods: A first degree consanguineous healthy parents -who have 3 deceased siblings with different pediatric cancers of an early onset- were subjected for genetic analysis using whole exome sequence analysis which was performed to detect germ line mutations that might be responsible for CMMRDs.

Results: Heterozygous Frame-shift mutation in PMS1 exone 5 was detected in both parents.

Conclusions: We concluded that this mutation-frame shift mutation in PMS1 exone 5 is likely culprit of disease in this family and maybe used in genetic counseling and screening of CMMRDs. The gene variant is to be added to the list of mismatch repaire gene mutation associated with the condition.

Reference: Adam R, Spier I, Zhao B et al Exome sequencing identifies biallelic MSH3 germline mutations as a recessive subtype of colorectal adenomatous polyposis. Am J Hum Genet(2016) 99(2):337-351.

Keywords: CMMRDs, conasguinity, PMS1, pediatric cancer.

\title{
Analysis of CFTR by next-generation sequencing in patients with cystic fibrosis from West
}

Olga Vladimirovna Romanova1, Tatyana Eduardovna Ivashchenk ${ }^{2}$, Oleg Sergeevich Glotov' ${ }^{1}$, Michael Andreevich Fedyakov ${ }^{1}$, Yuliya Almazovna Nasyhova², Anna Valer'yanovna Gorbunova ${ }^{3}$, Yury Aleksandrovich Barbitov ${ }^{4}, \mathrm{M}_{\text {Donnikov }}^{5}$, L Kolbasin ${ }^{5}$, Andrei Mikhaylovich Sarana ${ }^{1}$, Sergei Grigorievich Scherbak ${ }^{1}$, Vladislav Sergeevich Baranov ${ }^{2}$

1. St.Petersburg State Health Care Establishment the City Hospital ?40, St. Petersburg, Russian Federation

2. D.O.Ott Research Institute of Obstetrics, Gynecology and Reproductology, St. Petersburg, Russian Federation

3. Theodosius Dobzhansky Center for Genome Bioinformatics, St. Petersburg, Russian Federation

4. Saint Petersburg State University, Resource Centre Biobank, St. Petersburg, Russian Federation

5. Medical Genetics Counseling Service, Surgut, Russian Federation

Background: Cystic fibrosis (CF) is a common autosomal recessive disorder caused by CFTR mutations. CFTR encodes cystic fibrosis transmembrane conductance regulator protein.

Methods: We carried out the NGS analysis by "MiSeq" ("Illumina”, USA) in 35 patients with cystic fibrosis from West Siberia Siberia using “TruSight Inherited Disease" sequencing panel ("Illumina”, USA). Bioinformatic filtering of the DNA sequencing results was performed using “GeneTalk” (https://www.gene-talk.de/), “UGENE” (http://ugene.unipro.ru/), “Reporter Ion” (https://ionreporter.lifetechnologies.com/ir/), "SIFT” (http://sift.jcvi.org/) and "PolyPhen2” (genetics.bwh.harvard.edu/pph2/). Final results were verified by direct Sanger sequencing using ABI 3130 genetic analyzer (“Applied Biosystems”, USA).

Results: The most frequent mutation, delF508, was identified in 35\% of CF chromosomes. In two patients, we detected three mutations (instead of commonly presented two mutations): delF508/S466X/R1070Q. According to the literature, this variant S466X/R1070Q is a complex allele carrying a severe disease. The efficiency of the CF allele detection after NGS was 84,3\%. Eight novel mutations were found: c.T3983A (p.I1328K), c.43delC (p.L15fs), c.1580dupA (p.E527fs), c.T698G (p.L233R), c.T252A (p.Y84X), c.1845_1846delAA (p.L615fs), c.G1526A (p.G509D), c.G831A (p.W277X).

Discussion: NGS technology has a lot of advantages. However, one of the most important NGS disadvantages is an inability to identify large deletions and insertions. For example, large mutation CFTR21kbdel was detected in two patients only by PCR analysis. The efficiency of the $\mathrm{CF}$ allele detection may suggest the presence of the large CFTR deletions or insertions in patients with unknown genotype. Also we can use our data for comparison with NGS results of control groups for search of other variants of uncertain significance in patients with unknown genotype.

This study was supported by Russian Science Foundation №14-50-00069.

\section{POSTERS}

\section{Group 2 - PHARMACOGENOMICS}

\section{Study of CYP2D6 genotype-phenotype in a deprescription plan with prescription opioid dependence patients}

\author{
Ana María Peiró \\ Clinical Pharmaocology, General Hospital of Alicante, Alicante, SPAIN
}

Background: Opioids use in non-cancer pain is still controversial due to its safety profile can include prescription opioid dependence (POD). Our Pain Unit carries out an individualized deprescription plan in these cases where metabolic profile might affect the response to opioids or the vulnerability to develop dependence.

Objective: To analyze the influence of CYP2D6 phenotype in patients with chronic pain and POD. Design: Observational study following-up the IDP during 6 months with tapering of morphine equivalent daily doses (MEDD) and rotation to buprenorphine and tramadol ( $\mathrm{n}=120$ ). 
Pain intensity and relief, quality of life, opiate withdrawal syndrome, global activity and adverse events (AEs) information was collected. Patients were classified as responders to desprescription if a reduction of at least 30\% MEDD was achieved, without dependence behavior. Genetic analysis of CYP2D6 $\star^{\star} 2,{ }^{\star} 3{ }^{\star} 4,{ }^{\star} 6,{ }^{\star} 10,{ }^{\star} 17,{ }^{\star} 29,{ }^{\star} 35,{ }^{\star} 41(\mathrm{n}=67)$ was performed by real-time PCR, as well as number of copies, grouping as poor (PM), extensive (EM) or ultra-rapid (UM) metabolizers. The project was approved by Ethical Committee and analyzed by R 3.2.0. Results: Study population ( $53 \pm 13$ years old, $60 \%$ female) showed a moderate pain intensity and relief, with $71 \%$ of responders to desprescription. A total of 310 AEs were registered, with a median of 7 (4-9) per patient. Dry mouth (66\%) and sleep disruption (53\%) were the most frequent. Metabolic phenotype frequency was 6\% PM, 84\% EM and 10\% UM without any influence on clinical or drug variables. Different phenotypes frequency (PM, EM and UM) were found in AEs: headache (50, 33 and 100\%), dry mouth (0, 63 and 100\%) and depression (0,46 and $83 \%$ ) distribution.

Conclusions: UM-CYP2D6 phenotype chronic pain patients with OP, showed a different opioid security profile especially of neurology or psychiatric AEs.

Acknowledgements: Present study was partially funded by Spanish Pain Foundation Society, Grant Code: BF4-16-02.

References: 1. Zahari Z, Ismail R. Influence of Cytochrome P450, Family 2, Subfamily D, Polypeptide 6 (CYP2D6) polymorphisms on pain sensitivity and clinical response to weak opioid analgesics. Drug Metab Pharmacokinet. 2014;29(1):29-43

Keywords: opioid, dependence, pharmacogenetics, CYP2D6, metabolism

\section{A candidate SNP in the DPYD for precision Medicine in Breast Cancer chemotherapy among Sudanese}

Fatima Hamadto, Maha Othman, Ameera Adam and Muntaser Ibrahim

Molecular Biology, Institute of Endemic Diseases, Khartoum, Sudan

Background: Breast cancer (BC) is a major health problem in Sudan, and the death toll is believed to be aggravated in many cases by chemotherapeutic toxicity following treatment. Drug responses are known to differ between individuals, due to complex factors including genetic variations in drug metabolizing enzymes, transporters and targets. In Sudan the selection and administration of cytotoxic drugs follow the same guidelines regardless of the patients genetic profile and despite studies reporting high incidence of drug unresponsiveness among Sudanese BC patients. Genetic and precision medicine will help to improve therapeutic outcomes through identification and categorization of populations based on their pharmacogenetic profiles. In this study we start with the drug metabolizing enzymes variants that may relate to the action of 5-Fluorouracil Cyclophosphamid and epirubicin (FEC) family of drugs.

Objectives: To determine genetic variants affecting FEC chemotherapy outcome among Sudanese populations and allele frequency patterns of Single Nucleotide Polymorphisms (SNPs) associated with drug response in breast cancer patients.

Material and method: Exome sequence data of healthy Sudanese were retrieved from the Sudan Genome Project database at the Institute of Endemic Diseases (IEND) - University of Khartoum and analyzed using several bioinformatics tools to determine the SNPs profile of drug metabolizing enzyme genes used in breast cancer. This research had been approved by the ethics committee at IEND.

Result: Thirty Nine SNPs associated with the FEC drugs had been identified. Particularly DPYD SNP (rs1801265) that is known to be significantly associated with a decreased value of 5- flurouracil was detected with 0.7 frequency.

Discussion and conclusion: It is highly recommended that, before starting treatment with FEC breast cancer patients should be screened for rs1801265, a known SNP associated with decreased value of 5- flurouracil action and poor patient responsiveness, given its frequency in the population. This could be one of the early contributions of population based precision medicine in Sudan to improve patients response and avoid unnecessary drug usage. Further hospital based studies are required using large data sets.

References: Gentile G1,2, Botticelli A3, Lionetto L2, Mazzuca F3,4, Simmaco M1,2, Marchetti P3,4, Borro M1,2. Genotype-phenotype correlations in 5-fluorouracil metabolism: a candidate DPYD haplotype to improve toxicity prediction. The Pharmacogenomics Journal (2015), 1-6. Keywords: Precician Medicine

\section{The perils and possibilities of p53-based personalized cancer therapy}

$\underline{\text { Z H Siddik }}$, Guangam He, Xiaolei Xie

Department of Experimental Therapeutics, University of Texas MD Anderson Cancer Center, Houston, UNITED STATES

Background: Chemotherapy is an important modality cancer patient care, with the tumor suppressor p53 being a key player in apoptotic response and a successful therapeutic outcome. Activation of p53 is critical for not only conventional drugs such as cisplatin, but also rationally targeted therapeutics such as Gleevec. Unfortunately, loss of p53 function through mutation in about 50\% of all cancers leads to chemorefractory cancers, which limits the clinical care of cancer patients.

Objective: It may appear rational to stratify patients to receive personalized care based on mutational status of p53; however, substantial drug resistance in a number of cancers harboring the wild-type p53 gene limits the utility. In fact, this gene status is highly prevalent in refractory cancers, accounting for 35-90\% of resistant breast, non-small cell lung, and ovarian cancers. In some cases, such as high grade ovarian cancers, wild-type p53 can render cancers more resistant than mutant p53. Conversely, mutant p53 may retain function but can also be inactivated, as in 30-35\% of triple negative breast and colorectal cancers. Therefore, it is important to better understand the mechanisms of resistance in wildtype $\mathrm{p} 53$ and specific functional mutant $\mathrm{p} 53$ cancers and, thereby, rationalize treatment of such cancers for p53-based personalized therapy. 
Design: we have established a panel of cisplatin-resistant human ovarian tumor models harboring functional wild-type and/or mutant p53, and have examined the mechanism of resistance and its circumvention using molecular tools.

Results: We have demonstrated that absence of Chk2 kinase prevents cisplatin-induced DNA damage signals from phosphorylating p53 at serine-20 and activating p53 function. Alternatively, the oxaliplatin analog activates an alternative kinase, likely MEK1/2, and restores serine-20 phosphorylation and p53 function, upregulate apoptotic activity and circumvent drug resistance. In human ovarian cancer, we have demonstrated that although the p53-V172F mutant is functional, it is inactivated within the resistant tumor cells through binding to multiple molecules of the negative regulators MDM2 and MDM4, even though cellular basal MDM2 and MDM4 expressions are normal.

Conclusions: Our data provide a framework to permit the rational use of p53 function, rather than mutational status, as a more predictive biomarker in personalized medicine. However, additional clinical studies are needed for a definitive validation.

Acknowledgements: Studies were supported by grant NCI RO1-CA211975.

Keywords: p53, mutation, drug resistance, personalized therapy

\section{The Influence of CYP2D6 1846G>A Polymorphism on the Efficacy and Safety of Fluvoxamine in Patients with Depressive Disorder and Comorbid Alcohol Use Disorder}

Zastrozhin Mikhail ${ }^{1}$, Grishina Elena ${ }^{2}$, Savchenko Ludmila 3 , Bryun Evgeny ${ }^{4}$, Sychev Dmitry ${ }^{5}$

1. Addictology, Russian Medical Academy of Continuous Professional Education of the Ministry of Health of the Russian Federation, Moscow, Russian Federation

2. Research Center, Russian Medical Academy of Continuous Professional Education of the Ministry of Health of the Russian Federation, Moscow, Russian Federation

3. Russian Medical Academy of Continuous Professional Education of the Ministry of Health of the Russian Federation, Russian Medical Academy of Continuous Professional Education of the Ministry of Health of the Russian Federation, Moscow, Russian Federation

4. Addictology, Moscow Research and Practical Centre on Addictions, Moscow, Russian Federation

5. Clinical Pharmacology and Therapy, Russian Medical Academy of Continuous Professional Education of the Ministry of Health of the Russian Federation, Moscow, Russian Federation

Background: Fluvoxamine therapy is often ineffective and some patients suffer from dose-dependent adverse drug reactions, reducing the efficacy of the therapy of depressive disorder comorbid with alcohol use disorder. The presence of some polymorphic markers of CYP2D6 increases the amount of isoenzyme to be expressed or enhances its activity, and some polymorphisms reduce the isoenzyme activity resulting in the changes biotransformation and elimination rates of medication.

Objective: To investigate the effects of CYP2D6 genetic polymorphisms on the efficacy and safety of fluvoxamine in patients with depressive disorder and comorbid alcohol use disorder.

Methods: The study involved 113 male patients (average age: $35.13 \pm 8.65$ years) with depressive disorder and comorbid alcohol use disorder. A series of psychometric scales were used in the research. Genotyping of CYP2D6 (1846G >A) was performed using real-time polymerase chain reaction.

Results: According to results of U-test Mann-Whitney, statistically significant differences between the efficacy and safety of fluvoxamine were obtained on 9th and 16th days of therapy in patients with GG and GA genotypes (The Hamilton Rating Scale for Depression: 10.0 [10.0; 23.0 ] vs $25.0[24.0 ; 16.0](\mathrm{P}<0.001)$ on 9th day and $4.0[2.0 ; 5.0]$ vs $6.0[6.0 ; 7.0]$ on 16th day; Udvald for Kliniske Undersogelser Side Effect Rating Scale: $6.0[4.0 ; 6.0]$ vs $9.0[9.0 ; 10.0](\mathrm{P}<0.001)$ on 9 th day and $5.0[1.0 ; 9.0]$ vs $19.0[18.0 ; 22.0]$ on 16 th day.

Conclusion: This study demonstrated the lower efficacy and safety of fluvoxamine in patients with depressive disorder and comorbid alcohol use disorders with GA genotype in CYP2D6 1846G > A polymorphic marker.

References: Hicks JK, Bishop JR, Sangkuhl K, Müller DJ, Ji Y, Leckband SG, Leeder JS, Graham RL, Chiulli DL, LLerena A, Skaar TC, Scott SA. Clinical Pharmacogenetics Implementation Consortium (CPIC) Guideline for CYP2D6 and CYP2C19 Genotypes and Dosing of Selective S

\section{The interest of Therapeutic Drug Monitoring of Infliximab}

\section{Toumi Houari}

EHU Oran Department Pharmacovigilance, Ora, ALGERIA

The pharmaceutical industry develops an increasingly sophisticated biomedicines, which by their conception targets some specific pathological mechanisms, and have improved the management of various inflammatory diseases, for example chimeric anti-TNF $\alpha$ monoclonal antibodies such as Infliximab (IFX). However, the frequent occurrence of primary or secondary treatment failure, the high immunogenicity of this molecule, the problems of pharmacokinetic and pharmacodynamic variability as well as the strict conditions of interchangeability of its biosimilars make their use delicate. It is therefore recommended to determine for each patient the level of response to this treatment and to guide a therapeutic decision. So, we're talking about the Therapeutic Drug Monitoring (TDM).

The main objective of this work is to justify the interest of the TDM, to evaluate the effectiveness of the IFX, to analyze the predictive factors of treatment failure, to evaluate the tolerance by the detection of side effects and to estimate the cost of optimization strategies. For this purpose, 
a retrospective mono-centric prospective retrieval analysis was performed on the cohort of patients with IBD followed between 2012 and 2017 in the Hepato-gastroenterology department of the EHU Oran.

The cohort selected eight patients. Our correlation study of biological and clinical data, showed abnormally low values in remission or abnormally high thrust, suggesting that biological parameters are not strongly correlated with the effectiveness of IFX. In parallel, the study of the sensitivity confirmed these results. In our analysis, treatment failure occurred in $62.5 \%$ of cases after 3 to 16 months of treatment. Optimization was required in $37.5 \%$ of patients in our cohort. To avoid a failure, it was recommended to optimize 1 patient out of 3 under IFX. When applying this data to the additional cost of optimization strategies, the additional cost of the annual IFX treatment then ranges between 878970 and 5273820 Da per year. Thus, although these optimization strategies are identical to those of the literature, it is interesting to note that these strategies are taken at random and are the opposite of evidence-based medicine.

Our primary results justify the interest of the IFX's TDM to improve the management of patients on IFX. Further, prospective and randomized studies will be needed to demonstrate that the integration of this pharmacological parameter into the therapeutic strategy can modify the prognosis in the medium and long term of patients with IBD.

References: KIRCHGESNER J., Sokol H. Biological Explorations for Chronic Inflammatory Bowel Disease, Journal of gastroenterology MICI, leaflets of Biology / $\mathrm{N}^{\circ} 322$ - Jan 2015., BadreW., Biological markers during MICI. CHU Ibn Rochd-Casablanca. 2014, European Commission, What you need to know about biosimilars Process on corporate responsibility in the field of pharmaceuticals, Access to medicines in Europe A consensus document for information; Jun 2014, INESSS. Detection of Infliximab and Anti-Infliximab Antibody with ELISA or High Performance Liquid Chromatography: Notice of Assessment, Analysis from the Notice to the Minister dated February 28, 2014. (REFERENCE - 2013.03.009) Keywords: Biomedecines, Infliximab, Failure, Optimisation, Therapeutic drug monitoring, Cost.

\title{
COQ2 polymorphisms are not associated with increased risk of statin induced myalgia/myopathy in Czech population
}

Jaroslav Hubacek ${ }^{1}$, Vera Adamkova ${ }^{2}$, Lukas Zlatohlavek ${ }^{3}$, Lenka Steiner-Mrazova ${ }^{1}$, Michal Vrablik ${ }^{3}$

1. Department of experimental medicine, Institute for clinical and experimental medicine, Prague, Czech Republic

2. Department of preventive cardiology, Institute for clinical and experimental medicine, Prague, Czech Republic

3. 33rd Department of Internal Medicine, 1st Faculty of Medicine, Charles University, Prague, Czech Republic

Background: Gene COQ2, encoding 4-hydroxybenzoate-polyprenyltransferase (coenzyme Q2), belongs to the candidates potentially influencing statin treatment tolerability. This enzyme is involved in the biosynthesis of coenzyme Q10 (CoQ10), which depletion induced by statin treatment is implicated in the development of statin associated muscle symptoms (SAMS).

Objective: To analyse, if polymorphisms in the COQ2 gene might explain susceptibility to SAMS.

Design: Adult patients with SAMS (on low doses of atorvastatin and simvastatin) induced myalgia/myopathy ( $\mathrm{N}=278$ ), patients on statins but without SAMS $(\mathrm{N}=293)$ and population (part of the post-MONICA study) controls $(\mathrm{N}=561)$ were genotyped (PCR-RFLP assay) for rs6535454 and rs4693075 polymorphisms within the COQ2 gene.

Results: Distribution of the rs6535454 in patients with SAMS ( $G G=51.1 \%, \mathrm{GA}=40.0 \%, \mathrm{AA}=8.9 \%)$, did not significantly differ $(\mathrm{P}=0.33$; respectively 0.32 for codominant models of the analysis) from the population controls ( $\mathrm{GG}=48.1 \%$, GA $=45.0 \%$, AA $=6.9 \%$ ) or SAMS unaffected patients $(\mathrm{GG}=49.8 \%, \mathrm{GA}=40.4 \%, \mathrm{AA}=9.8 \%)$, Similarly neither rs4693075 was associated with $\mathrm{SAMS}(\mathrm{CC}=36.8 \%, \mathrm{CG}=48.2 \%$, $\mathrm{GG}=15.0 \%$ in patients suffering SAMS vs. $\mathrm{CC}=36.6 \%, \mathrm{CG}=47.5 \%, \mathrm{GG}=15.9$ in controls and $\mathrm{CC}=35.8 \%, \mathrm{CG}=48.2 \%, \mathrm{GG}=16.0 \%$ in symptom free patients, $\mathrm{P}=0.94$ and 0.95 for codominant models of the analysis). Also, the haplotype distributions were not significantly different between the groups analysed.

Conclusion: The polymorphisms of COQ2 gene do not associate with SAMS in the Czech patients treated with low doses of statins.

Acknowledgements: This work was supported by the project (Ministry of Health, Czech Republic) for the development of research organisation 00023001 (IKEM, Prague, Czech Republic) - Institutional support.

Keywords: CoQ, polymorphism, myopathy, statin

\section{Synthetic Switch-based Baculovirus for Transgene Expression Control and Selective Killing of Hepatocellular Carcinoma Cells}

\author{
Yu Cheng Andy \\ Chemical Engineering, NTHU, Hsinchu, Taiwan, Province of China
}

Baculovirus (BV) holds promise as a vector for anticancer gene delivery to combat the most common liver cancer-hepatocellular carcinoma (HCC). However, in vivo BV administration inevitably results in BV entry into non-HCC normal cells, leaky anticancer gene expression and possible toxicity. To improve the safety, we employed synthetic biology to engineer BV for transgene expression regulation. We first uncovered that miR-196a and miR-126 are exclusively expressed in HCC and normal cells, respectively, which allowed us to engineer a sensor based on distinct miRNA expression signature. We next assembled a synthetic switch by coupling the miRNA sensor and RNA binding protein L7Ae for 
translational repression, and incorporated the entire device into a single BV. The recombinant BV efficiently entered HCC and normal cells and enabled cis-acting transgene expression control, by turning OFF transgene expression in normal cells while switching ON transgene expression in HCC cells. Using pro-apoptotic hBax as the transgene, the switch-based BV selectively killed HCC cells in separate culture and mixed culture of HCC and normal cells. These data demonstrate the potential of synthetic switch-based BV to distinguish HCC and non-HCC normal cells for selective transgene expression control and killing of HCC cells.

Keywords: Baculovirus, HCC

\title{
Pharmacogenetic study of clopidogrel in Caribbean Hispanics
}

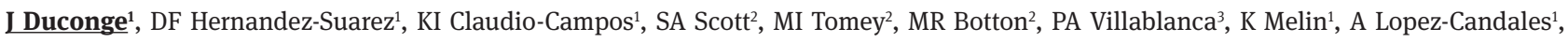 \\ JY Renta ${ }^{1}$ \\ 1. University of Puerto Rico Medical Sciences Campus, San Juan, Puerto Rico, United States; \\ 2. Icahn School of Medicine at Mount Sinai, New York, United States; \\ 3. Albert Einstein College of Medicine at New York, United States
}

Background: High on-treatment platelet reactivity (HTPR) with Clopidogrel is predictive of ischemic events in adults with coronary artery disease. Clopidogrel is the most commonly used P2Y12 inhibitor in Puerto Rico. Despite strong data suggesting HTPR varies with ethnicity, clinical and genetics, no pharmacogenetic studies of Clopidogrel have been performed in Puerto Ricans. Objective: This study was aimed to identify clinical and genetic predictors of HTPR in a cohort of Puerto Ricans with cardiovascular diseases.

Design: A pharmacogenetic association study was conducted in 130 Puerto Rican patients on Clopidogrel (75 mg/day). Patients were separated into two groups: Group I without HTPR and Group II with HTPR. Clinical data was obtained from medical record. Platelet function was measured by VerifyNow® P2Y12 assays and HTPR was defined as P2Y12 reaction units (PRU) 2230. Genotype testing for CYP2C19, ABCB1, PON1, PY2R12, PEAR1 and B4GALT2 were performed using Taqman ${ }^{\circledR}$ Genotyping Assays.

Results: Forty-two (38\%) patients had HTPR. Mean PRU was 203 \pm 61 . One in four individuals carried at least one copy of the CYP2C19*2 allele. Hematocrit and PON1 Q192R variant were inversely correlated with HTPR $(\mathrm{p}<0.05)$. Multiple logistic regression showed that 27\% of the total PRU variation was explained by history of diabetes mellitus (OR=3.46; CI: 1.05-11.43), hematocrit (OR=0.75; CI: $0.65-0.87$ ), CYP2C19^2 (OR=4.44; CI: 1.21-16.20) and PON1 Q192R alleles.

Conclusions: In a representative sample of cardiovascular patients from Puerto Rico, diabetes mellitus, hematocrit, CYP2C19^2 and PON1 Q192R variants were associated with HTPR. These factors may identify a subset of Hispanic patients at higher risk for adverse events.

Acknowledgements: This work was partially supported by awards CCTRECD-R25MD007607, HiREC-S21MD001830 and the Research Center in Minority Institutions (RCMI) award \#U54 MD007600 from the National Institute on Minority Health and Health Disparities (NIMHD), National Institutes of Health (NIH). Dr. Duconge is supported in part by the SC1 grant \#HL123911 from the National Heart, Lung and Blood Institute (HLBI) and the MBRS SCORE Program of the National Institute of General Medical Sciences (NIGMS) at NIH. Dr. Scott is supported in part by the grant K23GM104401 from NIGMS.

Keywords: Pharmacogenetics, Clopidogrel, Hispanics, Platelet Reactivity

\section{The highest joint prevalence of CYP2C9*2 and VKORC1 variants responsible for warfarin sensitivity in the Croatian Roma compared to 20 populations worldwide}

Tatjana Škaric-Juric, Branka Janicijevic, Nina Smolej Narancic, Matea Zajc Petranovic, Željka Tomas, Marijana Pericic Salihovic Institute for Anthropological Research, Zagreb, Croatia

Background: The genes encoding the cytochrome P450 2C9 enzyme (CYP2C9) and vitamin K-epoxide reductase complex unit 1 (VKORC1) are major determinants of anticoagulant response to warfarin1. Minor allele carriers of both loci respond in additive manner to a lower dose of warfarin, and they are at risk for adverse drug reaction when usual doses are applied. The Roma (Gypsy) are major European transnational minority population of Indian origin and long-lasting reproductive isolation that could result in specific genetic profile implying that the pharmacogenetic information could not just be extrapolated from surrounding populations.

Objective: To investigate the separate and joint minor allele frequencies (MAFs) of the two most important pharmacogenetic loci for warfarin dosage - VKORC1 (rs9923231) and CYP2C9*2 (rs1799853) - in the Roma population and to compare them with 20 populations worldwide.

Design: Both loci were genotyped in 422 Roma volunteers living in several regions of Croatia. The comparison is made with 20 populations worldwide (1000 Genomes data extended with the published data for the same countries).

Results: Locus rs9923231 has wide geographic variability with extremely high MAF among East Asian populations and almost zero in African populations. However, the rs1799853 MAF distribution does not follow the same global distribution since this allele is not present in East Asian populations, resulting with the highest joint minor alleles frequencies in Roma population amounting $10 \%$. They are followed by European (2.5-7.3\%) and South American (0.9-5.4\%), while in South Asian (0.3-1.0\%), East Asian (0-0.2) and African (0) populations the risk of combined findings of minor alleles at both loci is negligible or not existent. This finding is primarily result of the highest global MAF for rs1799853 in Croatian Roma (21.6\%) in combination with high MAF of rs9923231 in this population (46.1\%). 
Conclusions: Both VKORC1 (rs9923231) and CYP2C9*2 (rs1799853) minor alleles contribute to inter-population difference in adequate warfarin dosage. The present investigation points to the specific Roma population as the one with the highest frequency of combined MAFs at both loci. This puts them at high risk of adverse reaction when warfarin is applied without genetic profiling.

Acknowledgements: Croatian Science Foundation grant (HRZZ-IP-2014-09-4454)

References: 1 Fung E et al. Semin Thromb Hemostasis 2012;38:893-904.

Keywords: VKORC1, CYP2C9², warfarin, Roma population, MAF distribution

\section{Distinctiveness of the Roma population in pharmacogenetic "core list" markers within the worldwide populations}

Branka Janicijevic, Tatjana Skaric-Juric, Nina Smolej-Narancic, Matea Zajc Petranovic, Željka Tomas, Marijana Pericic Salihovic Institute for Anthropological Research, Zagreb, Croatia

Background: Proteins involving absorption, distribution, metabolism and excretion (ADME) of many endogenous and exogenous substances play an important role in determining the pharmacokinetics of drugs as well. There is a considerable variation in genes encoding ADME proteins, both within and between populations. Pharmacogenetic data on the isolated populations are rather scarce. Only few data are available on the prevalence of ADME gene variants in the Roma (Gypsy) transnational minority population of Indian origin (1).

Objective: We determine the allele frequencies of ADME "core list" markers and compare them with world-wide data in order to elucidate the position of Roma in the global perspective.

Design: The sample comprises 439 Croatian Roma from several regions of Croatia with the highest number of Roma minority individuals. Their DNA samples were genotyped for 95 loci from 32 ADME genes using KASP method. Data were analyzed by standard statistical population-genetics methods within the context of ethnically diverse populations from various parts of the world using the 1000 Genomes project and literature data.

Results: The analysis placed Croatian Roma among European populations but their proximity to South Asian populations is also evident suggesting that their ADME gene pool is a combination of ancestral (Indian) and more recent (European) layers. Due to their specific genetic history Roma show the outlying position on the global scale in minor allele frequencies of 12 loci: for 10 loci within 9 genes (rs1128503, rs1138272, rs1799853, rs1902023, rs3758581, rs8192709, rs10509681, rs12248560, rs34059508, rs28371725) they have the highest frequency while for two loci in two genes (rs28399433, rs4149117) the lowest frequency of the minor allele. According to the Clinical Pharmacogenetics Implementation Consortium - CIPC guidelines, rs1799853 (CYP2C9) and rs12248560 (CYP2C19) have been clinically annotated as level 1A supporting their high clinical importance.

Conclusion: Our results show that Roma population takes specific position within the global ADME genetic landscape and indicate necessity for the assessment of unique genetic profile of Roma in order to achieve the most in the modulation of pharmacotherapy in this population. Acknowledgements: The research was funded by Croatian Science Foundation grant (HRZZ-IP-2014-09-4454) to MPS.

References: Tomas Ž, Kuhanec A, Škaric-Juric T, Petranovic MZ, Naranci? NS, Janicijevic B, et al. Distinctiveness of the Roma population within CYP2B6 worldwide variation. Pharmacogenomics. 2017;18(17):1575-87.

Keywords: ADME, Roma, pharmacogenetics

\section{Long term metabolic improvements in morbid obese diabetic and non-diabetic patients after Roux-en-Y Gastric Bypass (RYGB): a stable isotope tracer study}

Chiara Barbieri ${ }^{1}$, Chiara Barbieri ${ }^{2}$, Fabrizia Carli ${ }^{1}$, Melania Gaggini ${ }^{1}$, Brenno Dominguez Brenno Dominguez ${ }^{4}$, Marianna Palumbo ${ }^{4}$, Ele Ferrannini $^{1}$, Stefania Camastra ${ }^{4}$, Amalia Gastaldelli ${ }^{1}$

1. Cardiometabolic Risk Laboratory, Institute of Clinical Physiology CNR, Pisa, Italy

2. Department of Biotechnology, Chemistry and Pharmacy, University of Siena, Siena, Italy

3. Cardiometabolic Risk Laboratory, Institute of Clinical Physiology CNR, PISA, Italy

4. Department of Clinical and Experimental Medicine, University of Pisa, Pisa, Italy

Background: Bariatric surgery, in particular Roux-en-Y Gastric Bypass (RYGB), leads to significant weight loss and improvement in glycaemic control, insulin resistance (IR) and beta cell function, and in many cases to the remission of type 2 diabetes (T2D) (1).

objective: The aim of this study was to evaluate if the effects RYGB, in particular improvement in glucose metabolism, were maintained 7 years later.

Design: The cohort comprised 16 patients (9 T2D and 7 non diabetic, ND). Patients were studied before surgery, 1 year and 7 years after RYGB using the infusion of stable isotope tracers of [6,6-2H]-glucose and [2H5]-glycerol to measure changes in endogenous glucose production (EGP), lipolysis (RaGlycerol) and insulin resistance (IR) indexes like hepatic (Hep-IR=(EGPxIns)) and adipose tissue (Lipo$\mathrm{IR}=($ RaGlycerolxIns)).

Results: We observed long term (7 ys) effects of RYGB in both ND and T2D not only in weight loss (BMI: $51.0 \pm 2.5$ basal, $36.4 \pm 2.31 \mathrm{y}$ and $39.5 \pm 7.87 \mathrm{ys}$ in ND vs $51.7 \pm 2.8$ basal, $33.9 \pm 1.9$ at $1 \mathrm{y}$ and $36.1 \pm 2.0$ at $7 \mathrm{ys}$ in T2D; $\mathrm{p}<0.0001$ ) but also in metabolic improvement, including diabetes remission in all T2D patients. Fasting glucose was significantly decreased at 1y (from 101.3 \pm 3.2 to $89.4 \pm 1.8$ in ND and $158.0 \pm 17.8$ to 
92.6 \pm 3.2 in T2D, $\mathrm{p}=0.0015)$ and maintained at $7 \mathrm{ys}(92.9 \pm 2.3$ in ND to $86.5 \pm 9.3$ in T2D; $\mathrm{p}=0.0007$ vs basal, $\mathrm{p}=\mathrm{ns}$ vs $1 \mathrm{y})$. This was associated with Hep-IR improvement at $1 \mathrm{y}$ (from $133.2 \pm 27.1$ to $53.1 \pm 8.8 \mathrm{in}$ ND and $177.4 \pm 44.6$ to $77.64 \pm 12$ in T2D, p < 0.05) was maintained at 7ys (46.7 \pm 9.9 in ND to $57.5 \pm 12$ in T2D; $p<0.05$ vs basal, $p=n s$ vs 1y). Lipo-IR decrease at $1 \mathrm{y}$ (from $68.7 \pm 24.6$ to $20.8 \pm 4.9$ in ND and $72.7 \pm 18.9$ to $26.8 \pm 5.3$ in T2D, $\mathrm{p}=0.009)$ was maintained at $7 \mathrm{ys}(17.3 \pm 5.2$ in ND to $19.2 \pm 3.9$ in T2D; $\mathrm{p}=0.0002$ vs basal, $\mathrm{p}=\mathrm{ns}$ vs $1 \mathrm{y})$.

Conclusions: RYGB not only dramatically reduces body weight but also improves IR, resulting in remission of diabetes already at $1 \mathrm{y}$ that is maintained 7ys after surgery.

Acknowledgements: this study received the support of grant from Ministry of Health \#RF-2011-02348446

References: 1. Camastra S, Gastaldelli A, Mari A, Bonuccelli S, Scartabelli G, Frascerra S, et al. Early and longer term effects of gastric bypass surgery on tissue-specific insulin sensitivity and beta cell function in morbidly obese patients with and without type 2,,

\section{Comparison study of CYP2C9 (rs1799853, rs1057910), CYP2C19 (rs4244285), CYP1A2 (rs762551), CYP11B2 (rs1799998), CYP17 (rs619824) frequency distribution between Southeastern European Caucasians and other populations}

Maria Papasavva, Danae Theodoridou, George Diles, Martha-Spyridoula Katsarou, Maria Lagiou, Thodoris Kalogridis, Nikolaos Drakoulis Research Group of Clinical Pharmacology and Pharmacogenomics, Faculty of Pharmacy, School of Health Science, National and Kapodistrian University of Athens, Athens, GREECE

Background: Important phase I drug metabolizing Cytochrome P-450 enzymes (CYPs) are involved in the metabolism of xenobiotics and endogenous substrates such as cholesterol, steroids and other lipids. Genetic polymorphisms within CYPs affect drug metabolism, resulting differences in drug response. CYP2C9 2 , CYP2C9 ${ }^{\star} 3$ and CYP2C19 2 are related with reduced enzyme activity, thus CYP1A2*1F is related with high enzyme activity. CYP11B2 polymorphism leads to increased aldosterone to rennin ratio.

Objective: A frequency distribution analysis of the polymorphisms CYP2C9^2 (rs1799853), CYP2C9*3 (rs1057910), CYP2C19^2 (rs4244285), CYP1A2*1F (rs762551), CYP11B2 (rs1799998) and CYP17A1 (rs619824) in a Southeastern European Caucasian (SEC) population was performed. Design: DNA was extracted by buccal swabs of 534 non related SEC's and genotyping was performed by real time PCR (Light Cycler 480, Roche, Germany) using appropriate LightSnip Assays (TIBMOLBIOL, Germany) and melting curve analysis. Statistical analysis was performed with IBM Statistics SPSS 22.0 package.

Results: Gene distribution for polymorphism rs 1799853 was $\mathrm{C}: \mathrm{C}=74,2 \%, \mathrm{C}: \mathrm{T}=23,2 \%$ and $\mathrm{T}: \mathrm{T}=2,6 \%$. The wild type $\mathrm{C}$ allele frequency was $85,8 \%$. Frequencies for polymorphism rs 1057910 were $\mathrm{A}: \mathrm{A}=83,9 \%, \mathrm{~A}: \mathrm{C}=15,7 \%$ and $\mathrm{C}: \mathrm{C}=0,3 \%$. The wild-type $\mathrm{A}$ allele frequency was $91,8 \%$. Frequencies for polymorphism rs 4244285 were $A: A=2,4 \%, A: G=23,0 \%$ and $G: G=74,5 \%$. The wild-type $G$ allele frequency was $86,0 \%$. Frequencies for polymorphism rs762551 were $\mathrm{A}: \mathrm{A}=49,8 \%, \mathrm{~A}: \mathrm{C}=43,1 \%$ and $\mathrm{C}: \mathrm{C}=7,1 \%$. The wild-type $\mathrm{C}$ allele frequency was $28,7 \%$. Frequencies for polymorphism rs619824 were $\mathrm{G}: \mathrm{G}=29,6 \%, \mathrm{G}: \mathrm{T}=50,0 \%$ and $\mathrm{T}: \mathrm{T}=20,4 \%$. The wild-type $\mathrm{T}$ allele frequency was $45,4 \%$. Frequencies for polymorphism rs 1799998 were $\mathrm{C}: \mathrm{C}=18,7 \%, \mathrm{C}: \mathrm{T}=48,5$ and $\mathrm{T}: \mathrm{T}=32,8 \%$. The wild-type $\mathrm{T}$ allele frequency was 57,0\%. For CYP2C9 2, CYP2C9 3 , CYP2C19 2 and CYP1A2 ${ }^{\star} 1 F$, statistically significant differences between Southeastern European Caucasian and African, American, East Asian and South Asian populations were observed, whereas, for CYP11B2 statistically significant differences between Southeastern European Caucasian and African, other European and East Asian populations were observed. For CYP17A1 statistically significant differences between Southeastern European Caucasians and African and East Asian populations were observed.

Conclusions: Adjustment of drug dosage should be taken into consideration, because of differences between populations.

Acknowledgements:This research was supported by the research group of Clinical Pharmacology and Pharmacogenomics at the National and Kapodistrian University of Athens.

\section{Identification of genetic changes relevant to radiation response using targeted next-generation DNA sequencing}

Eui Kyu Chie, Seung Hyuck Jeon, Yi-Jun Kim, Joo Ho Lee

Department of Radiation Oncology, Seoul National University College of Medicine, Seoul, Republic of Korea

Objectives: To demonstrate the feasibility of discovering gene or molecular pathway associated with radiation response with next-generation sequencing (NGS) data and radiologic assessment.

Methods: Targeted NGS data of 148 genes from biopsy samples of 55 cancer patients, who received radiotherapy (RT) to measurable target were analyzed. Patients with complete or partial remission lasting longer than 6 months after RT were considered as responders and otherwise non-responders. Mutations in the 148 genes of interest and 39 molecular pathways involving target genes were tested. Two analytic methods were employed; logistic regression model with interaction and Fisher's exact test.

Results: There were 17 responders and 38 non-responders. Only biologically equivalent dose (BED) was associated with radiation response among various clinical variables tested for significance $(\mathrm{p}=0.01)$. No single gene was correlated with radiation response with neither of the analytic methods. Using logistic regression model with interaction, ErbB signaling pathway was relevant to radiation response. However, it was not significant when multiple comparison was adjusted. Fisher's exact test revealed that mutation excluding non-pathogenic single nucleotide variations and copy number alterations in Notch signaling pathway resulted in radioresistance with statistical significance $(\mathrm{p}=0.002)$. When BED and Notch signaling pathway mutation were tested with logistic regression, both variables were associated with radiation response. 
Conclusions: This analysis showed that correlating genetic changes with radiation response is feasible with radiologic evaluation and targeted NGS. Notch signaling pathway may be associated with radiation response. The result of this study should be validated in larger and more homogenous cohort.

References: Yard BD, Adams DJ, Chie EK et al. Nat Commun 2016;7:11428, Yahyanejad S, Theys J, Vooijs M. Oncotarget 2016;7(7):7610-28. Keywords: radiotherapy, radiosensitivity, molecular pathway, next-generation sequencing

\section{Correlation of Polymorphisms in SCN1A Gene with Diagnosis and Effective Therapy of Focal Impaired Awareness Seizures}

Danae Tsikrika', Anastasia Verentzioti ${ }^{2}$, Panagiotis Patrikelis ${ }^{2}$, Nikolaos Drakoulis $^{1}$, Eleni Kokkiou ${ }^{1}$, Martha Katsarou ${ }^{1}$, Anna Siatouni ${ }^{2}$, Stylianos Gatzonis², Michael Koutsilieris ${ }^{3}$, Ioannis Sakkas³, Athanasia Alexoudi ${ }^{2}$

1. School of Health Sciences, National and Kapodistrian University of Athens, Research Group of Clinical Pharmacology and Pharmacogenomics, School of Pharmacy, Athens, Greece

2. School of Health Sciences, National and Kapodistrian University of Athens, A' Department of Neurosurgery, Medical School, Athens, Greece 3. School of Health Sciences, National and Kapodistrian University of Athens, Department of Experimental Physiology, Medical School, Athens, Greece

Background: Voltage-gated sodium channels are actively involved in epilepsy. SCN1A gene encodes the alpha subunit of the neuronal voltage-gated sodium channels in the brain, known as Nav1.1. and is the most important epilepsy related gene. Genetic polymorphisms in this gene have been involved in epileptogenesis and different epilepsy phenotypes. SCN1A protein also plays a major role as antiepileptic drug target.

Objective: The aim of this study is to investigate three common polymorphisms of SCN1A gene and their role both in drug responsiveness in antiepileptic therapy and susceptibility in Focal Impaired Awareness Seizures (FIAS).

Design: DNA samples from southeastern European Caucasians drug-responsive and drug-resistant epileptic patients with FIAS analyzed with real-time PCR for polymorphisms rs2298771, rs3812718 and rs10188577. Additionally, from Ensemble database, data from 503 individuals used as controls. Chi-square test used to evaluate the correlation of these polymorphisms with FIAS susceptibility and drug responsiveness. Samples were anonymized and de-identificated after an inform consent was signed by each patient.

Results: rs2298771 AA \& GG genotypes appear 2 times more often in epileptic patients with FIAS than in General population ( $p=0,003)$. Furthermore, individuals with a specific combination of polymorphisms of SCN1A gene seem to have different response to therapeutic antiepileptic agents. In this study, $60 \%$ of patients with genotypic combination of AA/TC/TT of rs2298771/ rs3812718/rs10188577, respectively responded well to therapy. In contrast, 80\% of patients with genotypic combination AG/CC/TT of rs2298771/ rs3812718/ rs10188577, respectively were poor responders to therapy.

Conclusions: There is a correlation between rs2298771 G allele and susceptibility in FIAS. Genotyping of SCN1A gene before initiating any anti-epileptic medication may contribute to personalization epileptic therapy of FIAS. Further investigation in larger groups of patients will validate these results.

Acknowledgements: This study supported by Research Group of Clinical Pharmacology and Pharmacogenomics at the National and Kapodistrian University of Athens, Greece.

\section{Association of CNS Genes OPRM1, DRD2 and BDNF Polymorphisms with Nicotine Addiction in a Southeastern European Population}

Anastasia Zoulia, Stavroula Nioma, Konstantinos Sarantidis, Eleni Kokkiou, Martha Katsarou, Maria Lagiou, Thodoris Kalogridis, Nikolaos Drakoulis

School of Health Sciences, National and Kapodistrian University of Athens, Research Group of Clinical Pharmacology and Pharmacogenomics, School of Pharmacy, Athens, Greece

Background: Nicotine is a highly addictive substance, which binds to CNS nicotine receptors (nAchRs) and regulates the release of neurotransmitters such as dopamine and $\beta$-endorphin. The OPRM1 gene encodes the mu opioid receptor, which is a target for $\beta$-endorphin. The DRD2 gene encodes the D2 receptor of dopamine. The BDNF gene encodes the protein neurotrophin, which plays an essential role in dopaminergic and GABA-ergic neurons' development and survival and may be involved in the pathway of nicotine inside the human body.

Objective: Purpose of this study is to investigate the polymorphisms rs1799971 (OPRM1), rs1800497 (DRD2) and rs6265 (BDNF) in association with nicotine addiction.

Design: DNA samples have been collected in buccal swabs from 314 Southeastern Caucasian (SEC) volunteers, who were then divided in two groups: smokers (109) and non-smokers (205). Genotyping was performed with real-time PCR (LightCycler 480, Roche) and appropriate LightSnip Assays (TIBMOLBIOL). All samples were anonymized and de-identificated after an inform consent was signed by each volunteer. 
Results: Among smokers, rs1799971 A:A genotype appears 34\% more frequent than mutant genotypes, rs1800497 C allele appears 23\% more frequent, while the rs6265 A:A mutant genotype is 3 times more frequent than the wild type. The three polymorphisms were evaluated both individually and in combinations.

Conclusions: These observations agree to previous studies that indicate rs1799971 A:A genotype, rs1800497 C allele and rs6265 A:A genotype to be linked with higher reward feeling while smoking and lower abstinence periods of smoking cessation. It seems there is a relation between the polymorphisms and nicotine addiction, but further investigation to larger populations is needed to validate these results.

Acknowledgements: This research was supported by the Research Group of Clinical Pharmacology and Pharmacogenetics at the National and Kapodistrian University of Athens, Greece.

All the authors report no conflict of interest.

\section{Pharmacogenetics of clopidogrel in coronary artery disease patients: Results from Turkish from clinical practice}

Cevik Mehtap ${ }^{1}$, Susleyici Belgin ${ }^{1}$, Ciftci Cavlan², Karaalp Atila³, Yurdakul Selen², Canbolat Ismail Polat ${ }^{2}$, Gezer Elifcan ${ }^{1}$, Bezci Kivanc ${ }^{1}$, Cagatay Penbe $^{4}$, Schaik Ron ${ }^{5}$

1. Molecular Biolgy, MarmaraUniversity, Istanbul, Turkey

2. Cardiology, Istanbul Bilim University, Istanbul, Turkey

3. Medical Pharmacology, Marmara University, Istanbul, Turkey

4. Biostatistics and Medical Science, Istanbul University, Istanbul, Turkey

5. Clinical Chemistry, Erasmus University Medical Center, Rotterdam, Netherlands

Background: Clopidogrel is among the most commonly prescribed antiplatelet drug used to prevent cardiovascular events in patients with acute coronary syndromes or a history of stroke, myocardial infarction, or peripheral arterial disease. Genetic variants are known to contribute significantly to variability between individuals in clopidogrel efficacy. Although multiple CYP enzymes are involved in clopidogrel metabolism, only genetic variations in CYP2C19 affect the clinical response to clopidogrel.

Objective: To determine whether CYP2C19 gene polymorphisms predicts Clopidogrel response in cardiovascular patients better than clinical factors such as age, body mass index, dose and medication co-ingestion in Turkish subjects with coronary artery disease.

Methods: Clopidogrel treated 54 patients ( 21 female, 33 male) aged between 44-85 were included in the study. For each patient the clinical data and drug-drug interactions were evaluated as a response to CYP2C19 star alleles. Demographic, biochemical and clinical characteristic of the patients were collected and related to CYP2C19 genotypes. Genomic DNA was isolated from peripheral blood using Qiacube with QIAamp DNA Mini Kit. For the detection of CYP450 2C19 star alleles, automated, multiplexing, continuous flow, random access microarray platform, the INFINITI-Plus System (Autogenomics) was used. For validation of the results, Real Time PCR was applied.

Results: Among the analyzed 54 coronary artery disease patients, 21 were found to be normal, 16 were detected as ultra, 16 were detected as intermediate and 1 was detected as poor metabolizer for Clopidogrel. Among the coronary artery disease patients under empirical therapy $29,7 \%$ were recommended to change the dose of the drug; $31,5 \%$ were recommended to change the drug whereas 38,8 \% were not recommended for drug or dose change. As a result in $61,2 \%$ of our study population, the current treatment has been recommended to be changed as a response to pharmacogenetic testing.

Conclusion: The obtained pharmacogenetic information for clopidogrel supports the idea that, genetic polymorphisms may contribute to variability in clinical decisions.

Acknowledgement: This study was supported by Istanbul Development Agency, Project No. TR10/16/YNY/0030.

\section{The impact of CYP2D6 mediated drug-drug interaction: a systematic review on a combination of metoprolol and paroxetine/fluoxetine}

Muh. Akbar Bahar ${ }^{1}$, Jasper Kampㄹ, Sander D Borgsteede ${ }^{2}$, Eelko Hak ${ }^{1}$, Bob Wilffert ${ }^{1}$

1. Unit of PharmacoTherapy, -Epidemiology \& -Economics, University of Groningen, Groningen, Netherlands

2. Dept. of Clinical Decision Support, Health Base Foundation, Houten, Netherlands

Background: The CYP2D6 substrate metoprolol is often prescribed in combination with the strong CYP2D6 inhibitors paroxetine or fluoxetine. This might cause a potential drug-drug interaction (DDI). However, the clinical relevance of this interaction is still controversial. Objective: The aim of the study is to evaluate the current available evidence for the clinical relevance of this drug-drug interaction. Design: Pubmed, Web of Science, Cochrane Library and Embase databases were used to systematically search for studies published before April 2018 reporting the effect of metoprolol and paroxetine or fluoxetine combination. The protocol of this study was prospectively registered in PROSPERO (CRD42018093087).

Results: We found 9 eligible articles which consist of 3 case reports, 2 observational and 4 experimental studies. All experimental studies consistently reported that paroxetine may increase the AUC of metoprolol to 3-7 times regardless of its dosage forms. Systolic blood pressure and heart rate of patients either during resting or after exercising were significantly decreased by the presence of paroxetine. Consequently, 
bradycardia and atrioventricular block may be triggered by the DDI as evidenced by the case reports. However, the available observational studies have conflicting results. A case control study presented that the interacting combination is not significantly associated with bradycardia compared to the non-interacting combination. In contrast, a cohort study indicated that the DDI is significantly associated with the incidence of early discontinuation of metoprolol as an indicator of the presence of metoprolol related side effects.

Conclusion: The present evidence shows contradictory results. However, there is an indication for the potential negative impact of the DDI. Therefore, since the alternative and safe antidepressants with comparable effectiveness are available, it is prudent to avoid the DDI. Nonetheless, if the combination is inevitable to be prescribed, a close monitoring of the metoprolol related side effects is a must especially in the high risk population.

Acknowledgement: Muh. Akbar Bahar has obtained a DIKTI scholarship from the Ministry of Research, Technology and Higher Education of Indonesia.

Keywords: CYP2D6; paroxetine/fluoxetine; metoprolol; drug-drug interaction

\section{Mutated cyclin dependent kinase 4 and Retinoblastoma 1 may promote chemo-resistance to palbociclib in ovarian cancer}

Said El Shamieh ${ }^{1}$, Fatima Saleh ${ }^{1}$, Fadi Farhat ${ }^{2}$

1. Medical Laboratory Sciences, Beirut Arab University, Beirut, Lebanon

2. Hematology and Oncology, Saint Joseph University, Beirut, Lebanon

Worldwide, ovarian cancer (OC) is the eight leading cause of cancer mortality among women. This high mortality rate of OC is mostly attributed to its late detection, poor prognosis, recurrence and emergence of drug resistance. Due to the profound heterogeneity of OC at the clinical, cellular and molecular levels, in the current study, we discuss the molecular findings at protein and genetic levels seen in our patient diagnosed with $\mathrm{OC}$, which could serve as a basis for prediction of effective response to personalized treatment.

Immunohistochemistry showed a complete loss of phosphatase and tensin homolog (PTEN), this observation was the reason behind prescribing palbociclib IBRANCE®, a drug with anti-tumor properties in PTEN deficient malignancies. However, there was no response to treatment. Next-generation sequencing analysis was performed to understand the basis of chemo-resistance, showing a missense and a nonsense mutation; p.E184K and p.R552X in cyclin dependent kinase 4 (CDK4) and retinoblastoma 1 (RB1) genes respectively. While p.E184K in CDK4 decreases the binding to cyclin D and p16, p.R552X in RB1 also decrease the binding to E2F, two events that will induce cell cycle progression and thus be responsible for the chemo-resistance to Palbociclib.

Keywords: Ovarian cancer, Next generation sequencing, mutations, personalized therapy

\section{Pharmacogenomics and Phenotypic Alterations as Predictive Biomarkers of Fluoropyrimidine-based Chemotherapy Toxicity}

Roberto Michela, Arrivi Giulia, Romiti Adriana, Lionetto Luana, Botticelli Andrea, Di Pietro Francesca Romana, Mazzuca Federica, Simmaco Maurizio, Marchetti Paolo

Department of Oncology, Sant’Andrea Hospital - Sapienza University of Rome, Rome, Italy

Introduction: Prediction and early management of severe toxicity might avoid both therapy's interruption and the benefit loss of adjuvant chemotherapy. However, predictive biomarkers are not yet available. The aim of this study was to investigate whether polymorphisms of different genes involved in fluoropyrimidine metabolism and the phenotypic test, 5-fluorouracil (5-FU) degradation rate, were associated with the outcome of oral fluoropyrimidine-based adjuvant chemotherapy in patients with early stage gastrointestinal (GI) cancer.

Methods: Genotyping of DPYD IVS14 IVS 14+1 G>A, MTHFR C677T and A1298C SNPs were performed by pyro-sequencing technology. PCR analysis was used for genotyping TYMS-TSER. Using PBMC cells, we also evaluated the 5-FU degradation rate, which determines the amount of drug consumed by cells in a time unit. Patients were categorized in two groups according to their value of 5-FU degradation rate: below the 5th centile (poor metabolism - PM) or above the 95th centile (ultra-rapid metabolism - UM) and within 5-95th centile (0.85-2.2 ng/ml $/ 106 \mathrm{cells} / \mathrm{min})$. Results: One hundred forty-two patients with early stage colon (39\%), rectal (28\%), stomach (20\%) and pancreatic (13\%) cancer, treated with 5FU-based adjuvant monochemotherapy, was included in this retrospective analysis. Forty-three per cent of patients had a lymphnodepositive disease, and 37\% received concomitant radiotherapy. Most of patients had an ECOG PS $=0-1$. Seventy-four and 20\% of the patients suffered from at least one G1-4 and G3-4 adverse events (12 hematologic, 24 GI, 12 Hand-Foot Syndrome), respectively. Sixteen (11\%) patients resulted abnormal 5-FU metabolizers. At a multivariate logistic regression analysis, an altered 5-FU degradation rate $(<0.86>2.10)$ resulted significantly associated with both G1-4 hematologic $(\mathrm{OR}=2.99,95 \% \mathrm{CI}$ 0.98-9.12, $\mathrm{P}=0.05)$ and all grade $3-4$ adverse events $(\mathrm{OR}=4.39,95 \% \mathrm{CI}$ $1.40-13.80, \mathrm{P}=0.01)$. No correlation was reported between toxicity and each tested gene polymorphism.

Conclusions: Our study showed a statistically significant association between 5-FU degradation rate and both G1-4 hematologic and G3-4 toxicities. Therefore, the 5FU-degradation rate may be considered as a putative, predictive biomarker of fluoropyrimidine-related toxicity. 
References: Mazzuca F, Borro M, Botticelli A, et al. Pre-treatment evaluation of 5-fluorouracil degradation rate: association of poor and ultrarapid metabolism with severe toxicity in a colorectal cancer patients cohort. Oncotarget. (2016),,,

Implementation of a pharmacogenetic panel test service and its potential clinical impact on medication costs in Finland

Juho Heliste ${ }^{1}$, Klaus Elenius ${ }^{1}$, Varpu Elenius ${ }^{2}$

1. Institute of Biomedicine, University of Turku, Turku, Finland

2. Department of Paediatrics and Adolescent Medicine, Turku University Hospital, Turku, Finland

Background: Utilization of pharmacogenetics in the clinics is increasing due to decreased costs by testing multiple genes in singular panel tests. However, there is lack of feasible clinical decision support systems to support clinicians. Knowledge about the clinical impact of pharmacogenetic panel testing would improve the clinical use of pharmacogenetics and lower the medical costs.

Objective: To address potential clinical impact of pharmacogenetic testing by studying genotype and phenotype frequencies and affected annual medication costs in Finland.

Methods: 159 healthy volunteers were genotyped with a real-time RT-PCR-based SNP genotyping chip for 12 genes and with an additional assay testing for CYP2D6 copy number variations. Genotypes were interpreted to diplotypes and corresponding phenotypes. Gene test reports were generated automatically with an interpretation software, utilizing a gene-drug recommendation database, both produced by Abomic Ltd. Phenotype distribution information was used to estimate the extent of significantly affected prescriptions and reimbursements in Finland annually, based on reimbursement data by The Finnish Social Insurance Institution Kela [1].

Results: 95\% of individuals was found to carry at least one significant pharmacogenetic variant. High frequency (6.5\%) of CYP2D6 ultrarapid metabolizers was found in the Finns compared to other Caucasians as reported before. Extrapolated from the phenotype frequencies, approximately 380,000 significantly pharmacogenetically affected prescriptions are made in Finland annually. Total reimbursement costs of these potentially problematic or uneffective medications are 28 million euros per year.

Conclusions: The pharmacogenetic test panel service was found to be functional, and coverage of only 12 genes in the test was found to have potentially high clinical impact on prescribed medications nationwide.

Acknowledgements: We thank Auria Biobank for providing the genotype data and Abomics' technical staff for generating the reports.

References: Official Statistics of Finland: Reimbursements of medicine expenses: Number of recipients and prescription data [Internet]. Helsinki: Kela. 2018 [cited 06/18/2018]. Available from: http://raportit.kela.fi/ibi_apps/WFServlet?IBIF_ex=NIT137AL\&YKIELI=E

\section{Real-world outcomes in patients with metastatic melanoma treated with anti-PD1 agent nivolumab}

Ekaterina Shakhray ${ }^{1}$, Igor Samoylenko', Galina Kharkevich¹, Natalia Petenko ${ }^{1}$, Yaroslav Zhulikov², Lev Demidov ${ }^{1}$

1. Biotherapy, N.N. Blokhin Russian Cancer Center, Moscow, Russian Federation

2. Department of general medicine, I.M. Sechenov First Moscow State Medical University of the Ministry of Health of the Russian Federation, Moscow, Russian Federation

Background: New agents were approved for the treatment of metastatic melanoma (MM): BRAFi/MEKi, PD-1 and CTLA-4 inhibitors. Data of their use in treatment-naive and well selected MM pts is well presented. We analyzed the efficacy and safety of anti-PD-1 agent nivolumab within the real clinical practice of the single institution.

Methods: We conducted the retrospective study to assess the safety and efficacy of nivolumab in the 2nd and subsequent lines for MM treatment. Previously treated MM pts who received at least 1 administration of nivolumab $3 \mathrm{mg} / \mathrm{kg}$ were included in the analysis.

Results: 53 pts who received nivolumab in our institution were identified. Mean age was 52.8 y (95\% CI 23-76), male were 35.8\% pts, ECOG status 0 - in 39.6\% pts, stage M1c - in 71.7\% pts, LDH >ULN - in 37,7\% pts, CNS metastases - in 20.8\% pts, BRAF mutant tumors - in 54.7\%, cutaneus melanoma - in $84.9 \%$ pts, history of adjyuvant therapy - in $60.4 \%$ pts, 3 and more previous lines of therapy - in $47.2 \%$ pts, history of ipilimumab - in $66.0 \%$ pts. PDL status was not routinely defined.

Objective response rate (RECIST 1.1) was 22.6 (95\% CI 53.3-64.4) with CR 13.7\%. The median of duration of the response was not reached with median follow up time $30.7 \mathrm{mo}$.

Median PFS was 4.37 mo (95\% CI 2.27-6.47), median OS was 17.9 mo (95\% CI 8.89-26.99)

The subsequent therapy received $47.2 \%$ (including BRAFi/MEKi in $22.6 \% \mathrm{pts}$ ).

Negative prognostic value on OS in patients treated with nivo had LDG $>$ ULN vs LDG $<$ ULN, ECOG $=1,2$ vs ECOG = 0, sum of diameters of the target lesions $>100 \mathrm{~mm}$ vs $<100 \mathrm{~mm}$, progressive disease vs disease control (as the best overall response). Differences in patients characteristics, achieved disease control vs progressie disease are summurized in table.

AEs (all grades) were registered in 58,5\% with Grade 3-4 AEs in 13,2\%. In 5,6\% (3) pts AEs lead to treatment discontinuation. No unexpected AEs were described. 
Conclusion: our data suggest that OS and ORR are numerically lower in 2nd and subsequent lines in the real life clinical practice than in well selected patients participating in clinical trials. Nonetheless the CR rate of $14 \%$ is stable despite the setting of nivolumab administration. Keywords: Metastatic melanoma, nivolumab, PD-1

\section{Association between MTHFR C677T gene polymorphisms and cardiovascular adverse events in patients with metastatic colorectal cancer treated with 5 -fluorouracil based chemotherapy}

Arrivi Giulia, Roberto Michela, Di Pietro Francesca Romana, D’Antonio Chiara, Milano Annalisa, Macrini Serena, Marchetti Paolo, Mazzuca Federica

Clinical and Molecular Medicine Department, S Andrea Hospital, Sapienza University of Rome, Medical Oncology Unit, Rome, ITALY

Introduction: Methylentetrahydrofolate reductase (MTHFR) is a key enzyme regulating intracellular folate levels, which affect DNA synthesis and methylation. It has been hypothesized that tumors exhibiting deficient MTHFR variants such as 677T may be more sensitive to 5-fluorouracil (5-FU) cytotoxicity than tumours bearing the common MTHFR variants 677C (1). Moreover this gene polymorphism has also been considered as potential factor for 5-FU toxicity (2). Our study investigates MTHFR C677T gene polymorphism in patients affected with metastatic colorectal cancer (mCRC) treated with chemotherapy in association with bevacizumab.

Patients and methods: From November 2012 to June 2014 were enrolled in this study 54 patients with metastatic colorectal cancer receiving first-line chemotherapy with Folfiri-Bevacizumab. All patients were studied with total body CT scans and clinical responses were evaluated according to RECIST criteria, while toxicity according to CTCAE v4.0. Peripheral blood samples were collected from each patients and genomic DNA was extracted from white blood cells (WBC) for testing C677T polymorphisms.

Results: High-grade cardiovascular toxicity did not occur in forty-four (about 81\%) patients. Instead, ten (18\%) patients experienced G3-4 cardiovascular toxicity (hypertension, arterial and venous thrombotic events): one patient (2\%) showed $677 \mathrm{C} / \mathrm{C}$ polymorphism, three patients $(5 \%)$ the $677 \mathrm{C} / \mathrm{T}$ one and six patients $(11 \%)$ the $677 \mathrm{~T} / \mathrm{T}$ one. From these data we can observe that patients bearing the MTHFR $677 \mathrm{~T} / \mathrm{T}$ mutation reported G3-4 cardio-vascular adverse events more frequently than both 677C/T and 677C/C genotypes (p-value $<0,01$ ). Overall response rate (ORR) was 41\%: 1 (2\%) complete response (CR), 21 (39\%) partial response (PR), 18 (33\%) stable disease (SD) and 14 (26\%) disease progression (PD). A clinical significant association between polymorphisms and clinical responses was observed in this study: patients with $677 \mathrm{C} / \mathrm{T}$ and $677 \mathrm{~T} / \mathrm{T}$ mutations showed an ORR $=46 \%$ compared with $23 \%$ in $677 \mathrm{C} / \mathrm{C}$ genotype $(\mathrm{p}=0.13)$.

Conclusion: These data suggest an important role of MTHFR polymorphisms in predicting cardio-vascular adverse events in patients with mCRC treated with 5-fluorouracil based chemotherapy and bevacizumab. Further studies are required to better define the role of such polymorphism in clinical practice.

\section{References:}

1. Etienne-Grimaldi MC, Francoual M, Formento JL, Milano G., Methylenetetrahydrofolate reductase (MTHFR) variants and fluorouracil-based treatments in colorectal cancer, Pharmacogenomics. 2007

2. Thomas F1, Motsinger-Reif AA, Hoskins JM, Dvorak A, Roy S, Alyasiri A, Myerson RJ, Fleshman JW, Tan BR, McLeod HL., Methylenetetrahydrofolate reductase genetic polymorphisms and toxicity to 5-FU-based chemoradiation in rectal cancer, Br J Cancer. 2011

Keywords: 5-fluorouracil,MTHFR, polymorphisms, cardiovascular

\section{Rare variants in CYP2C9 and CYP3A5 detected in patients with familial hypercholesterolemia with statin-related adverse events}

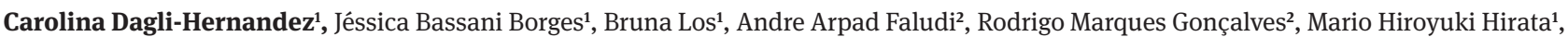
Rosario Dominguez Crespo Hirata ${ }^{1}$

1. School of Pharmaceutical Sciences, University of Sao Paulo, Sao Paulo, Brazil

2. Institute Dante Pazzanese of Cardiology, Sao Paulo, Brazil

Background: Statins are the first-choice treatment for primary dyslipidemias, including familial hypercholesterolemia (FH), but some patients experience statin-related adverse events (SRAE), which can result in low adherence to therapy.

Objective: To investigate variants in pharmacokinetics-related genes in FH patients with SRAE.

Design: FH patients $(n=26)$ with molecular diagnosis based on MEDPED criteria were selected at the Institute Dante Pazzanese of Cardiology, Brazil. Clinical, pharmacotherapy, SRAE and therapy adherence data were obtained from medical records. CYP2C9, CYP3A4 and CYP3A5 genes were sequenced using MiSeq platform (Illumina). Primary, secondary and tertiary data analyses were performed using Real Time Analysis, MiSeq Reporter, BaseSpace Sequence Hub and VariantStudio. In silico functional prediction analyses were conducted using Polyphen-2, SIFT and PROVEAN.

Results and Discussion: SRAE was reported by 38.5\% HF patients. Four missense variants were identified in CYP2C9 and CYP3A5 genes in 7 patients with SRAE and no variants were found in CYP3A4. CYP2C9 3 (rs1057910, 1000 Genomes MAF: 4.8\%), which causes low enzyme activity, was detected in 3 patients, 2 of which reported atorvastatin-associated myopathy and 1 reported atorvastatin-associated kidney pain. CYP2C9 c.752A>G (rs2256871, 1000 Genomes MAF: 2.20\%) was detected in 3 patients, 2 of which reported atorvastatin-associated myopathy 
and 1 showed low adherence to atorvastatin, possibly due to unreported myopathy. CYP3A5 c.299C>A (rs41279857, 1000 Genomes MAF: 0.06\%) was identified in a patient that reported simvastatin-associated myopathy. In silico functional prediction analyses showed that both rs2256871 and rs41279857 variants are possibly modifying the enzymes' function, suggesting CYP2C9 and CYP3A5 loss-of-function. The reduced activity of both enzymes could increase statin levels, which could explain the occurrence of SRAE and possibly result in low adherence to therapy. Conclusions: CYP2C9 and CYP3A5 rare variants may be related to statin-related adverse events in FH patients. Acknowledgements: FAPESP (\#2016/12899-6) and CNPq (\#447120/2014-0).

Keywords: statin, myalgia, next-generation sequencing, adverse drug events, pharmacogenetics

\title{
Genetic association study to identify biomarkers suitable for individualized dug therapy for diabetic and hypertensive patients in South Africa
}

Mongi Benjeddou ${ }^{1}$, Charity Masilela ${ }^{1}$, Bredon pearce ${ }^{1}$, October Zainonesa ${ }^{1}$, Joven Jebio Ongole ${ }^{2}$

1. Biotechnology department, University of the Western Cape, Cape Town, South Africa

2. Family medicine, Piet Retief Hosptial, Mkhondo, South Africa

Background: Pharmacogenomics is playing an increasingly important role in the drug development process and the rational pharmacotherapeutic management of patients. Pharmacogenomic profiling has the potential to increase the efficacy of commonly used therapeutics, while greatly reducing adverse drug reactions. Over the past few years, genetic polymorphisms in proteins involved in drug metabolizing enzymes and transporters have been identified as clinically relevant, explaining differences in efficacy and impacting clinical care [1]. This is what makes pharmacogenomics one of the core elements of personalized (precision) medicine. In the case of diabetes and hypertension, there are several examples of genes in the literature and databases with relatively strong data on associations of genetic polymorphisms with anti-diabetic and anti-hypertensive drugs' response. Several genetic variants for the response to treatment with anti-diabetic and anti-hypertensive drugs have been identified. These genetic variants can be used in the selection and optimization of treatment for hypertension and diabetes. Objective: The development of an individualised drug therapy for diabetic and hypertensive patients in South Africa.

Methods: Outpatients with type 2 diabetes (T2DM) and hypertension and taking diabetic and hypertensive drugs as monotherapy or combined therapy were recruited for the study from the public and private hospitals. The cohort of patients is divided into two groups; patients with controlled and patients with uncontrolled diabetes and hypertension following the treatment with diabetic and hypertensive drugs. Genetic association studies are performed to investigate the effect of SNP variants and haplotypes on responsiveness to drug therapy for diabetes and hypertension.

Results: Important pharmacogenomics data for the relevant SNPs for Metformin and Glibenclamide therapy for type 2 diabetes, and Hydrochlorothiazide and Amlodipine therapy for hypertension were obtained. These include the level of association between genetic variants and response to therapy for patients.

Conclusion: The data generated in the project can be used by participating clinicians to individualise therapies for their patients in terms of dug choice and dose.

References: Pacanowski M and Huang SM, Precision medicine. Clin. Pharmacol. Ther. 99: 124-129, 2016.

Keywords: pharmacogenomics, diabets, hypertension, South Africa

\section{Oral Alpha-Cyclodextrins for the Disease-Modifying Treatment and the Prevention of Age-related diseases (including Cancer, Atherosclerosis, Alzheimer's / Parkinson's disease, and Multiple Sclerosis)}

\author{
Knut M. Wittkowski ${ }^{1,2}$ \\ ${ }^{1}$ The Rockefeller University, New York, NY, USA; \\ ${ }^{2}$ ASDERA LLC, New York, NY, USA
}

Background: The ability of lysosomes to degrade cellular waste declines with age, yet the phosphoinisitol (PI) cycle, which drives endocytosis, does not. Depending on mutations affecting endocytic trafficking, lysosomal function, and autophagy (ELA), different types of waste accumulate, contributing to many age-related diseases: carcinomas (integrins), atherosclerosis (LDL), Alzheimer's and Parkinson's disease (A $\beta /$ tau/aSyn), and multiple sclerosis (myelin). Treatments aiming to improve lysosomal function have not shown to be effective.

Objective: To identify novel interventions for improving ELA.

Design: We conducted independent analyses of three breast cancer (BCa) public GWAS followed up by in vitro and human PK/PD studies. Results I: The GWAS results consistently confirmed receptor signaling and nuclear function as involved, but also pointed to genes underlying "derailed", "deranged”, or "dysfunctional” endocytosis. The same genes have been seen in functional studies of BCa, CAD, and AD/PD, confirming a common etiology across age-related conditions.

Conclusions I: Cyclodextrins are rings of sugars, which form hydrophilic toroids with a lipophilic cavity. Beta-cyclodextrins (bCD, 7 sugars) are effective in Neymann-Pick disease, an early-onset lysosomal cholesterol storage disease, and in animal models of several age-related 
diseases, but scavenging cholesterol was soon seen to causing permanent hearing loss. The GWAS results, however, point to gain-of-function (GOF) mutations in genes transporting phospholipids (PLs) into the PI-cycle. Like bCDs, alpha-cyclodextrin (aCD, 6 sugars) also scavenges PLs, but does not fit cholesterol.

Results II: Hydroxypropyl (HP)aCD was more than twice as effective than HPbCD in reducing migration of human BCa cells.

Conclusion II: aCD emerges as a safer and more effective treatment of diseases where bCD was effective in vivo. CDs, however, are not absorbed from the intestine.

Results III: A PK study showed a novel aCD compound (pat. pending) to be absorbed. From the PD results, HPaCD scavenges only phosphatidylcholine (PC), but no lyso-PLs. Hence, HPaCD also reduces production of arachidonic acid, thereby, like NSAIDs, inflammation.

Conclusion III: The oral HPaCD compound emerges as a treatment, but also a prevention of age-related diseases, including carcinomas, $\mathrm{CAD}, \mathrm{AD} / \mathrm{PD}$, and MS ("baby-aspirin for baby-boomers") in people with mutations in PL transporters and/or genes involved in endocytosis, lysosomal function, and autophagy.

Reference: Wittkowski KM, Dadurian C, Seybold MP, Kim HS, Hoshino A, Lyden D. PLOS ONE. 2018;13:e0199012. 